\title{
Towards Building Energy Efficient, Reliable, and Scalable NAND Flash Based Storage Systems
}

\author{
A Dissertation \\ Presented to \\ the Faculty of the School of Engineering and Applied Science \\ University of Virginia
}

In Partial Fulfillment

of the requirements for the Degree

Doctor of Philosophy

by

Vidyabhushan Mohan

December 2015 


\section{Approvals}

This dissertation is submitted in partial fulfillment of the requirements for the degree of Doctor of Philosophy

Vidyabhushan Mohan (Author)

This dissertation has been read and approved by the Examining Committee:

\begin{tabular}{c}
\hline Kevin Skadron (Advisor) \\
\hline Jack Davidson (Chair) \\
\hline
\end{tabular}

Jiwei Lu

Mircea Stan

Marty Humphrey

Accepted by the School of Engineering and Applied Science:

Craig H. Benson (Dean)

December 2015 


Abstract

NAND Flash (or Flash) is the most popular solid-state non-volatile memory technology used today. As the memory technolgy scales and costs reduce, flash has replaced Hard Disk Drives (HDDs) to become the de facto storage technology. However, flash memory scaling has adversely impacted the energy efficiency and reliability of flash based storage systems. While smaller flash geometries have driven storage system capacity to approach petabyte limit, performance of such high capacity storage systems is also a major limitation. In this dissertation, we address the power, reliability, and scalability challenges of NAND flash based storage systems by modeling key metrics, evaluating the tradeoffs between these metrics and exploring the design space to build application optimal storage systems.

To address the power efficiency of flash memory, this dissertation presents FlashPower, a detailed analytical power model for flash memory chips. Using FlashPower, this dissertation provides detailed insights on how various parameters affect flash energy dissipation and proposes several architecture level optimizations to reduce memory power consumption.

To address the reliability challenges facing modern flash memory systems, this dissertation presents $F E N C E$, a transistor-level model to study various failure mechanisms that affect flash memories and analyze the trade-off between flash geometries and operation conditions like temperature and usage frequency. Using FENCE, this dissertation proposes both firmware level algorithms to enable reliable and application optimal storage systems.

Finally, to address scalability limitations of flash based high capacity Solid State Disks (SSDs), this dissertation evaluates the bottlenecks faced by conventional SSD architectures to show that the size of the indirection tables and the processing power available in such architectures severely limit 
performance as SSD capacity approaches the petabyte limit. This dissertation proposes FScale, a scalable, distributed processor based SSD architecture that can match the scaling rate of NAND flash memory and enable high performance petabyte scale SSDs. 


\section{Legal Notice}

\section{Chapters 2, 3, and 4, Copyright (C) 2015 Vidyabhushan Mohan}

All other chapters, Copyright (C) 2015 SanDisk Corp

Except for Chapters 2, 3, and 4, this dissertation and all material and know-how it contains including my dissertation and any and all Exhibits and Annexes thereto (collectively "the Dissertation".) were developed entirely at and constitute proprietary information of SanDisk. Subject to the University of Virginia's right to archive and to make available my Dissertation in whole or in part through the University's library in all forms of media, SanDisk reserves and retains all rights, title and interest in the Dissertation. 


\section{Acknowledgments}

This dissertation would not have been possible without the help of many people and it will be incomplete without acknowledging them.

Of all of them, I want to first thank my advisor Professor Mircea Stan. Mircea has lent a strong hand of support and helped me navigate this journey which started in 2008. I'm grateful for freedom that he has given me to explore ideas on my own and by allowing me to finish graduate studies even though I was off-grounds for a significant time duration. He has always been there to listen to me and provided valuable feedback to improve the quality of my work.

I want to thank my co-advisor, Professor Kevin Skadron. Discussions with Kevin have taught me how to construct a problem statement, think clearly, remove the noise and find answers to the core questions. His feedback has helped me to improve my presentation style and his insightful comments have helped me to solidify my research ideas. I want to thank my committee members Professor Jack Davidson, Professor Marty Humphrey and Professor Jiwei Lu for serving in the committee and for their guidance. I also want to thank Professor Sudhanva Gurumurthi for providing financial aid and advising me during my formative years in graduate school.

I want to thank Jack Frayer, one of the committee members and my manager at SanDisk. Jack has been a constant source of support, encouraging me to come up with new ideas and helping me focus on bringing those ideas to life. I want to thank Dr. Yale Ma and Nima Mokhlesi, for having confidence and hiring me even before I completed graduate studies. I also want to thank SanDisk for providing me the opportunity to work on cutting edge research and the support to continue my education. I would like to thank Dr. Hongzhong Zheng and James Tringali for mentoring me and providing the opportunity to contribute to exciting research projects as an intern. 
At UVa, I formed many new friendships that I hope will last for this lifetime. Ram's presence and support has helped me stay positive, seek out and cherish hard challenges and appreciate the meaning of life. He made me feel at home, inspired me to become a long distance runner and taught me to remain equanimous in victory and defeat. Many thanks to my roommates, Balaji, Abhishek and Avinash for putting up with me for many years and cheering me up after a very long day at work. I will forever cherish the late night Taco Bell dinners with Avinash and the passionate debates with Balaji. Thanks to Chih-hao, Tanima, Enamul, Wei, Munir, Vijay, Anurag and Rukmani for making graduate school memorable. Special thanks to Sriram for introducing me to UVa, being a good friend and a collaborator. My sincere thanks to Taniya for being the ideal team member. Her tenacity and work ethic inspired me and her presence made our a lab a cheerful place. Without her and Munir, I wouldn't have had the rare opportunity to drive cross country. I'm forever thankful to have a friend like her.

I would like to thank my undergraduate advisor, Professor Ranjani Parthasarthi for sowing the seeds of interest in the field of computer architecture. She encouraged me (and many of my classmates) to explore this exciting field and to express ourselves. During tough times, I often turned to my dear friend TS, who was always willing to provide a shoulder to lean on and let me catch some breath.

This dissertation would not have been possible without the love, sacrifice, faith and prayers of my parents. I cannot fathom their unwavering faith in my abilities. Amma and appa have cheered every single success of mine, and upon each failure lifted me on to their shoulders and kept me going. Life would be very dull without my sister who has always supported me. Between us, she is the real doctor - someone who can find a cure for a disease and save people's life. My wife has been a great source of strength throughout this journey. She has remained extremely patient, always encouraged me and managed our family as I spent many nights and weekends tucked in my office working on completing this dissertation. Watching our son grow has been revitalizing and keeps reminding me of the virtues of unbrindled excitement and joy in learning new things. Many other family members have wished for my success and have gone out of their way to ensure that I succeed. I'm forever grateful to be part of such a family. 
Finally, thanks to the almighty for helping me complete this dissertation successfully.

Krishnarpanam asthu. 


\section{Contents}

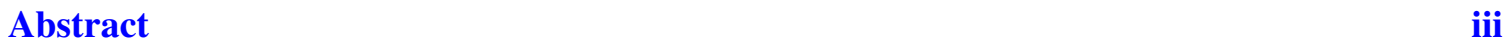

Legal Notice $\quad$ v

Acknowledgments vi

1 Introduction 1

1.1 Major Contributions . . . . . . . . . . . . . . . . . 3

1.1.1 Analytical Model for NAND flash Energy Dissipation . . . . . . . . . . 3

1.1.2 Architecting Reliable Solid State Storage Devices . . . . . . . . . . . . . . 3

1.1.3 Architecture Petabyte Scale SSDs . . . . . . . . . . . . . . 4

2 Understanding NAND Flash Energy Consumption 5

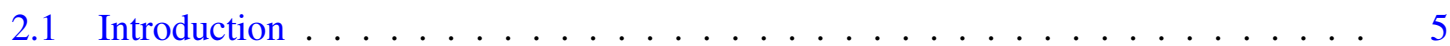

2.2 Microarchitecture of NAND Flash Memory $\ldots \ldots \ldots \ldots$

2.2.1 Components of NAND Flash Memory . . . . . . . . . . . . . . . 6

2.2.2 Basic Flash Operations . . . . . . . . . . . . . . . . . . . 8

2.2.3 Architectural Layout of Flash Memory _ . . . . . . . . . . . . . . 9

2.3 FlashPower: A Detailed Analytical Power Model . . . . . . . . . . . . . . . . . . 10

2.3.1 Circuit Components . . . . . . . . . . . . . . . . . 10

2.3.2 Power State Machine . . . . . . . . . . . . . . . . . . . . . . . 11

2.3.3 Extending FlashPower for n-bit MLC flash . . . . . . . . . . . . . . . . 14

2.3.4 Integration with CACTI $\ldots \ldots \ldots \ldots \ldots$ 
2.3.5 Power Modeling Methodology . . . . . . . . . . . . . . . . . . . 15

2.3.5.1 Modeling the Parasitics of an FGT . . . . . . . . . . . . 15

2.3.5.2 Derived Parameters . . . . . . . . . . . . . . 17

2.3.5.3 Transition from the Powered Off to the Precharged State . . . . . 18

2.3.5.4 The Read Operation . . . . . . . . . . . . . . . . . . . 19

2.3.5.5 The Program Operation . . . . . . . . . . . . . 21

2.3.5.6 The Erase Operation . . . . . . . . . . . . . . . . . 24

2.3.5.7 Charge pumps ................... 27

2.3.5.8 Multi-plane operation . . . . . . . . . . . . . . 27

2.4 Experimental Setup . . . . . . . . . . . . . . . . . . 28

2.4 .1 Hardware Setup . . . . . . . . . . . . . . . . . . . 28

2.4 .2 Test Procedure . . . . . . . . . . . . . . . . 28

2.5 Validation of FlashPower . . . . . . . . . . . . . . . . . . . 30

2.5.1 Validation for SLC flash . . . . . . . . . . . . . 32

2.5.2 Validation for 2-bit MLC flash . . . . . . . . . . . . . . 33

2.6 Design Space Exploration Using FlashPower . . . . . . . . . . . . . . . 36

2.7 Related Work . . . . . . . . . . . . . . . . . . . . . . . . . . . 39

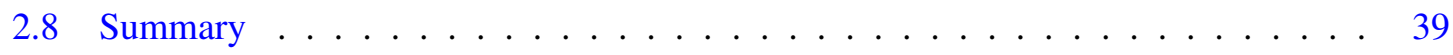

3 Modeling NAND Flash Memory Reliability 41

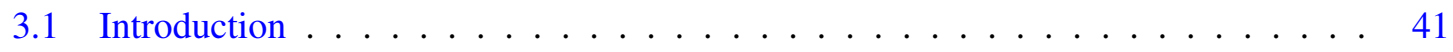

3.2 Overview of Flash Reliability . . . . . . . . . . . . . . . . . . . . 42

3.2.1 Factors affecting Flash Reliability . . . . . . . . . . . . . . . . 42

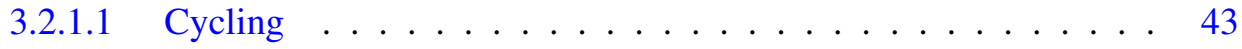

3.2.1.2 Recovery Period . . . . . . . . . . . . . . . 43

3.2.1.3 Temperature ..................... . . 44

3.3 An Analytical Model for NAND Flash Data Retention . . . . . . . . . . . . 45

3.3.1 Model for Data Retention _ . . . . . . . . . . . . . . . . . 46 
3.4 Impact of Detrapping on Data Retention . . . . . . . . . . . . . . . . . 48

3.5 Impact of Temperature on Data Retention . . . . . . . . . . . . . . . 50

3.6 Related Work . . . . . . . . . . . . . . . . . . . . . . 52

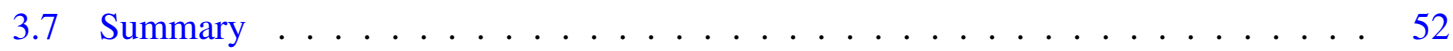

4 Building High Endurance, Low Cost SSDs for Datacenters 54

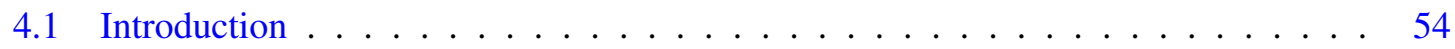

4.2 Experimental Methodology . . . . . . . . . . . . . . . . 57

4.2.1 SSD Configuration and Simulator Setup _ . . . . . . . . . . . 57

4.2 .2 Workloads ............................ 58

4.3 Simulating SSDs with HDD Workload Traces . . . . . . . . . . . . . . . 58

4.4 Estimating SSD Lifetime Over Long Timescales . . . . . . . . . . . . . . . 60

4.5 Impact of SSD Workloads on MLC Flash Reliability . . . . . . . . . . . . . 63

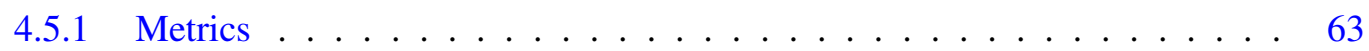

4.5.2 Baseline Evaluation . . . . . . . . . . . . . . . . 64

4.5.3 reFresh SSDs - An alternative approach to increase SSD lifetime . . . . . 65

4.6 Related Work . . . . . . . . . . . . . . . . . . . . . . 70

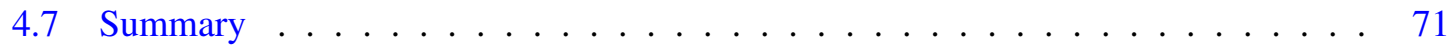

5 Architecting Petabyte Scale SSDs $\quad 72$

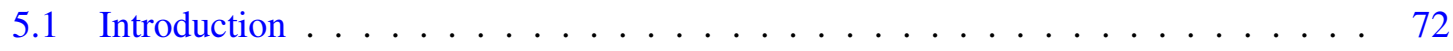

5.2 Overview of Enterprise SSD Architecture . . . . . . . . . . . . . . 74

5.3 Modeling Conventional SSD Architectures . . . . . . . . . . . . 75

5.3.1 Modeling Methodology _. . . . . . . . . . . . . . 75

5.3.1.1 Hardware Model . . . . . . . . . . . . . . . 76

5.3.1.2 Flash Translation Layer (FTL) Model . . . . . . . . . . . . . 76

5.3.1.3 Modeling FTL Latency . . . . . . . . . . . . . . 77

5.3.2 Modeling Accuracy and Relevance . . . . . . . . . . . . . . . . . 79

5.4 Scalability Challenges in Conventional SSD Architectures . . . . . . . . . . 80 
5.4.1 Metrics, Workloads . . . . . . . . . . . . . . . . . . 80

5.4.2 L2P Table Size and Cache Capacity . . . . . . . . . . . . . . . . 81

5.4.2.1 Drawbacks in existing solutions . . . . . . . . . . . 83

5.4 .3 SSD Controller Utilization . . . . . . . . . . . . . . . . . . . . . 83

5.4.4 Challenges in Scaling FTL Processor Performance . . . . . . . . . . . . . 85

5.4.5 Host Interface Processor, ECC Engine and NAND . . . . . . . . . . . . 86

5.5 FScale: A Scalable SSD Architecture f . . . . . . . . . . . . . . 88

5.5.1 FScale Hardware Architecture . . . . . . . . . . . . . . . . 88

5.5.1.1 FScale Hardware Architecture Assumptions . . . . . . . . . . . 89

5.5.2 FScale FTL Architecture . . . . . . . . . . . . . . . . . . . . . . 89

5.5.2.1 Top-level FTL . . . . . . . . . . . . . . . . . . . . . . 990

5.5.2.2 Back-end FTL . . . . . . . . . . . . . . . . . . . 90

5.6 FScale Performance Results ． . . . . . . . . . . . . . . . . . . . . . . . 91

5.6.1 L2P Table Size and Cache Capacity . . . . . . . . . . . . . . . . 91

5.6 .2 FScale Performance Analysis _. . . . . . . . . . . . . . . 93

5.6.3 Impact of Workload Queue Depth on FScale Performance . . . . . . . . 95

5.6.4 Other Advantages of FScale Architecture . . . . . . . . . . . . . . . . . . 97

5.7 Related Work . . . . . . . . . . . . . . . . . . . . . . . . . 97

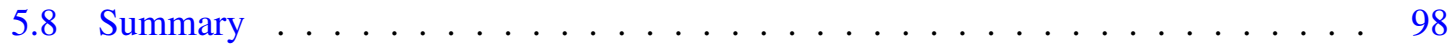

6 Conclusions and Future Work 99

6.1 Emerging Non Volatile Memories and Evolution of Memory Hierarchy . . . . . . . 101

$\begin{array}{ll}\text { Bibliography } & 103\end{array}$ 


\section{List of Figures}

2.1 Circuit Layout of a NAND Flash Memory Plane. . . . . . . . . . . . . . . 7

2.2 Power State Machine for an SLC NAND Flash Chip . . . . . . . . . . . . . . 11

2.3 Power State Machine for a 2-bit MLC NAND Flash Chip . . . . . . . . . . . . . 12

2.4 Transition between MLC Program States. . . . . . . . . . . . . . . . . . . . . 13

2.5 Modeling a Floating Gate Transistor . . . . . . . . . . . . . . 17

2.7 Read Energy Comparison for SLC flash . . . . . . . . . . . . . . . . . 31

2.8 Program Energy Comparison for SLC flash $\ldots \ldots \ldots \ldots$

2.9 Erase Energy Comparison for SLC flash . . . . . . . . . . . . . . . . 32

2.10 Read Energy Comparison for MLC flash . . . . . . . . . . . . . . . 34

2.11 Program Energy Comparison for MLC flash . . . . . . . . . . . . . . 35

2.12 Erase Energy Comparison for MLC flash f . . . . . . . . . . . . 36

3.1 Trapping and Detrapping in Floating Gate Transistors . . . . . . . . . . . . . 44

3.2 Impact of Recovery Periods on SLC and MLC Flash Data Retention . . . . . . . . 49

3.3 Impact of Temperature on SLC Data Retention $\ldots \ldots \ldots$. . . . . . . . 50

3.4 Impact of Temperature on MLC Data Retention . . . . . . . . . . . . . . . . 51

4.1 Cost Analysis of SSDs in Datacenters f . . . . . . . . . . . . 56

4.2 Impact of Varying Workload Intensity on SSD Response Time $\ldots \ldots \ldots$

4.3 Impact of SSD Workload on M-SSD Reliability . . . . . . . . . . . . . 65

4.4 Impact of EDF Refresh Algorithm On the Lifetime of M-SSD $\ldots \ldots \ldots$

5.1 Conventional SSD Architecture. . . . . . . . . . . . . . . . . . . 74 
5.2 FTL Operation Types and Phases . . . . . . . . . . . . . . . . . . 78

5.3 L2P Table Size as a function of SSD Capacity and Logical Page Size . . . . . . . . 80

5.4 Random Read Throughput as a Function of SSD Capacity . . . . . . . . . . . 81

5.5 SSD Controller Utilization as a Function of Drive Capacity and Workload Write

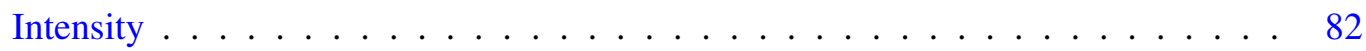

5.6 Random Write Throughput as a Function of SSD Capacity . . . . . . . . . . 85

5.7 FScale Hardware Architecture . . . . . . . . . . . . . . . . . . . . 87

5.8 FScale FTL Architecture . . . . . . . . . . . . . . . . . . . . . . 87

5.9 L2P Table Size Comparison Between Conventional and FScale Architecture . . . . 92

5.10 Workload Performance with FScale Architecture . . . . . . . . . . . . . . 94

5.11 FScale Performance at Various Queue Depths . . . . . . . . . . . . . 96 


\section{List of Tables}

2.1 Address Map for a SLC Block. . . . . . . . . . . . . . . . . . . . . . 9

2.2 Address Map for a 2-bit MLC Block. . . . . . . . . . . . . . . . . 9

2.3 Inputs to FlashPower $\ldots \ldots \ldots \ldots \ldots \ldots \ldots$

2.4 NAND flash chips used for evaluation $\ldots \ldots \ldots \ldots \ldots \ldots$

2.5 Different $16 \mathrm{~Gb}$ Chip Configurations . . . . . . . . . . . . . . . . 37

2.6 Energy dissipation for various $16 \mathrm{~Gb}$ Chip Configurations. . . . . . . . . . . . . . 37

2.7 Energy dissipation for various bit patterns for X1-M16 . . . . . . . . . . 38

4.1 Properties of Enterprise Workloads Used for Evaluation. . . . . . . . . . . . . 58

4.2 Cross validation results for extrapolated vs measured parameters. . . . . . . . . . 62

5.1 Major parameters for the SSD architecture model. . . . . . . . . . . . . . . . 79

5.2 Comparing Cache Performance Metrics with Conventional and FScale Architecture 92 


\section{Chapter 1}

\section{Introduction}

We are currently in the era of data centric computing where an increasingly interconnected population has been generating data at a pace and scale far beyond Moore's law. As the volume of data generated increases, there is a significant need for storage devices that can not only store data persistently but also retrieve them reliably, quickly and in an energy efficient manner. NAND Flash (or Flash) based storage systems are a class of persistent storage devices that use transistors connected together in a NAND topology to store and retrieve data. Common examples of such storage devices include Universal Serial Bus (USB) drives, Secure Digital (SD) cards, and Solid State Drives (SSDs) used in laptops, workstations and servers. Unlike other class of storage devices (like Hard Disk Drives (HDDs) and tape), flash based storage devices do not contain any mechanical components to store and retrieve data making them smaller, faster, robust and energy efficient. As the physical and logical dimension of flash memory scales and the cost of memory reduces, the adoption rate of flash has continued to increase. In order to facilitate this growth, system architects are required to optimize the storage systems for specific metrics like power, reliability and performance. While some of the optimizations can be employed at the memory chip level, others require system level changes to enhance system metrics. The overall goal of this dissertation is to address the power, reliability and scalability challenges of NAND flash based storage systems by modeling the metrics, evaluating the tradeoffs between the metrics and exploring the design space to build application optimal storage systems.

Power is an important consideration for flash memories because their design is closely tied to the 
power budget of the system within which they are allowed to operate. Given that flash memories are used in a wide range of systems, an accurate knowledge of power consumption and insights into how power is consumed in flash are critical. There has been little research on how various parameters affect energy dissipation as most studies rely on worst case estimates from data sheets. The first objective of this dissertation is to dissect the internal architecture of flash memory chips and build an analytical model of its energy dissipation. We then use the model to attain insights on flash energy dissipation and propose architecture optimizations that can reduce the power consumption of these memories.

NAND flash based storage systems face multiple reliability challenges. The cell-structure and organization of transistors inside the chips coupled with high current required for reading and writing from these transistors affects their reliability significantly. As the flash transistor technology scales down in size, the reliability issues faced by the technology are greatly exacerbated. Considering the wide disparity in the reliability requirement of various storage devices, the second objective of this dissertation is to model important failure mechanisms that affect NAND flash based storage and propose firmware and architecture level solutions that can mitigate these failures to design application optimal storage systems.

Current storage system architectures and Error Correction Code (ECC) mechanisms have enabled the industry to scale storage capacity by from the gigabytes to terabytes. Over the past 10 years, the capacity of NAND based storage systems have increased by three orders of magnitude and are expected to approach a petabyte within the next decade [22]. As memory scaling continues, existing system architectures should also scale accordingly to manage hundreds of NAND flash chips while providing optimal performance, reliability and energy efficiency. The third and final objective of this dissertation is to build an architecture level model to demonstrate the challenges in scaling current SSD architectures. In order to overcome these challenges, this dissertation proposes a new architecture that can effectively scale with NAND capacity and provide an optimal storage system behavior. 


\subsection{Major Contributions}

The specific contributions of this dissertation are as follows.

\subsubsection{Analytical Model for NAND flash Energy Dissipation}

We present FlashPower, a detailed power model for the two most popular variants of NAND flash namely, the Single-Level Cell (SLC) and 2-bit Multi-Level Cell (MLC) based NAND flash memory chips. FlashPower models the key components of a flash chip during the read, program, and erase operations and is highly parameterized to facilitate the exploration of a wide spectrum of flash memory organizations. We validate FlashPower against power measurements from chips from various manufacturers and show that FlashPower estimates are comparable to the measured values. We present case studies to show that FlashPower can be used for designing power optimal NAND flash memory chips for use in storage systems. This work has been published in Design Automation and Test in Europe (DATE), 2010 and IEEE Transaction on Computer Aided Design(TCAD), 2013 $[58,59]$

\subsubsection{Architecting Reliable Solid State Storage Devices}

We present FENCE, an analytical model to understand the factors affecting NAND flash reliability. Using the model, we show the impact of recovery period and temperature on NAND flash data retention. We use FENCE to explore the relationship and trade-offs between two dominant flash failure mechanisms namely: endurance and data retention. We also use FENCE to show how to trade-off data retention to increase endurance for NAND flash based storage devices used in data centers. We propose firmware algorithms that exploit the trade-off to increase storage device endurance from $6 \%$ to $56 \%$ depending on the enterprise workload while employing mechanisms to prevent data loss. This work has been published as a tech report and presented in Flash Memory Summit, 2012 [97]. 


\subsubsection{Architecture Petabyte Scale SSDs}

We study the performance of conventional enterprise SSDs by building a detailed architecture model. Using data collected from real enterprise SSDs, we model individual hardware resources inside the SSD controller and the interaction between the software (Flash Translation Layer) and hardware resources. Using this model, we explore the scalability limitations of conventional enterprise SSDs and conclude that the size of Logical-to-Physical (L2P) address translation table and the compute power in these SSDs severely limit their performance. Our results indicate that as SSD capacity scales towards the petabyte limit, the performance degrades by more than $30 \%$ indicating that existing architectures have poor scalability. To address these deficiencies, we propose FScale, new distributed processor based SSD architecture designed to scaling enterprise SSD performance with capacity. FScale hardware architecture comprises of many programmable and low power controllers named Local Controllers that are designed to manage a NAND flash package. We evaluate the performance of FScale architecture as a function of SSD capacity using a detailed model and show that unlike conventional architectures, SSD performance can increase with capacity by up to $3 \mathrm{X}$ using the FScale architecture compared to conventional SSDs of the same capacity. We propose a 2-level hierarchical L2P table scheme that works with FScale architecture and efficiently uses the cache memory available in the SSDs. We show that the proposed L2P mapping scheme improves the cache hit rate by up to $4 \mathrm{X}$ resulting in improved SSD performance.

The rest of this dissertation is organized as follows: In chapter 2, we present FlashPower, a detailed analytical power model for NAND flash memories. Chapter 3 presents FENCE, a reliability model for NAND flash memories. In chapter 4 we use FENCE to demonstrate the trade-offs between different flash failure mechanisms to build high endurance Solid State Disks (SSDs) for

use in data centers. In chapter 5, we discuss the challenges in architecting petabyte scale SSDs and propose a new architecture to overcome these challenges. Chapter 6 concludes this dissertation. 


\section{Chapter 2}

\section{Understanding NAND Flash Energy Consumption}

\subsection{Introduction}

Quantifying the energy consumption of flash accurately is necessary for many reasons. Power is an important consideration because the design of flash memories is closely tied to the power budget within which they are allowed to operate. For example, flash used in consumer electronic devices has a signicantly lower power budget compared to that of a SSD used in data centers, while the power budget for flash used in disk based caches is between the two. In such scenarios, an accurate knowledge of flash power consumption is beneficial. Insights into flash power consumption are also necessary because hybrid memories use flash in conjunction with other new non-volatile memories like Phase Change RAM and Spin-Transfer Torque RAM [45, 87]. Power aware design of such hybrid memory systems is possible only when an accurate estimate of flash energy consumption is available. So far, most studies that quantify the energy consumption of flash were limited to using datasheets. But research has shown that such datasheet-derived estimates are too general and do not take the intricacies of flash memory operations into account. Datasheets only provide average energy estimates (which in certain cases can deviate from the actual behavior by nearly $3 \mathrm{X}$ ) and cannot be used when accurate estimates are required. These inaccuracies arise chiefly because they cannot account for properties like workload behavior that have an significant impact on flash energy consumption. In particular, we lack simulation tools that can accurately estimate the power consumption of various flash memory configurations. 
To address this void, we present FlashPower, a detailed power model for the two most popular variants of NAND flash namely, the Single-Level Cell (SLC) and 2-bit Multi-Level Cell (MLC) based NAND flash memory chips. FlashPower models the key components of a flash chip during the read, program, and erase operations, and when idle, and is highly parameterized to facilitate the exploration of a wide spectrum of flash memory organizations. FlashPower is built on top of CACTI [100], a widely used tool in the architecture community for studying memory organizations, and is suitable for use in conjunction with an architecture simulator. First, we validate FlashPower against power measurements from chips from various manufactures and we show that FlashPower estimates are comparable to the measured values. We then illustrate the versatility of FlashPower through a design space exploration of power optimal NAND flash memory array configurations.

The organization of the rest of this chapter is as follows. Chapter 2.2 provides an overview of the microarchitecture and operation of NAND flash memory. Chapter 2.3 presents the details of the power model. Chapter 2.4 explains the experimental setup while Chapter 2.5 compares the results from FlashPower with actual chip power measurements from a variety of manufacturers. Chapter 2.6 presents a design space exploration using FlashPower. Chapter 2.7 presents the related work and Chapter 2.8 summarizes the work.

\subsection{Microarchitecture of NAND Flash Memory}

In this section, we describe the components in a NAND flash memory array, how they are architecturally laid out and their operation.

\subsubsection{Components of NAND Flash Memory}

Flash is a type of EEPROM (Electrically Erasable Programmable Read-Only Memory) that supports read, program, and erase as its basic operations. A NAND flash memory chip includes command status registers, a control unit, a set of decoders, some analog circuitry for generating high voltages, buffers to store and transmit data, and the memory array. An external memory controller sends read, program or erase commands to the chip along with the relevant physical address. The main 
component of a NAND flash memory chip is the flash memory array. A flash memory array is organized into banks (referred to as planes). Figure 2.1 describes a flash plane. Each plane has a page buffer (composed of sense-amplifiers and latches) which senses and stores the data to be read from or programmed into a plane. Each plane is physically organized as blocks and the blocks are composed of pages. A controller addresses a flash chip in blocks and pages. Thus a plane is a two dimensional grid composed of rows (bit-lines) and columns (word-lines). At the intersection of each row and column is a Floating Gate Transistor (FGT) which stores a logical bit of data. In this dissertation, the terms "cell" and "FGT" refer to the same physical entity and are used interchangeably. In Single-Level Cell (SLC) flash, the FGT stores a single logical bit of information, while in Multi-Level Cell (MLC) flash, the FGT stores more than one bit of information. Even though the term MLC flash means the number of bits stored in a FGT can be more than 1, we use the term MLC flash mostly in the context of a 2-bit MLC flash.

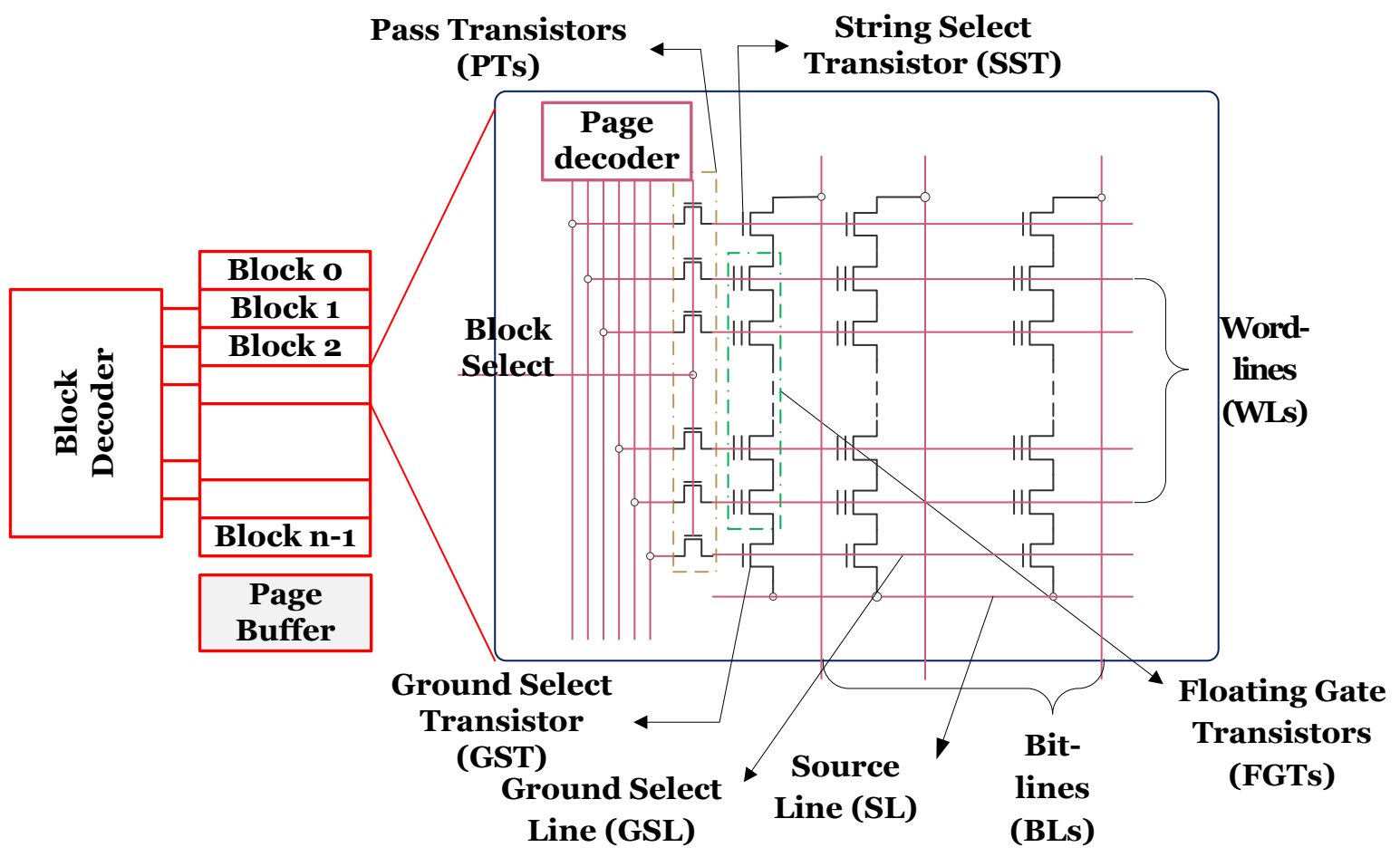

Figure 2.1: Circuit Layout of a NAND Flash Memory Plane.

A group of bits in one row of a plane constitute a page. Each NAND flash block consists of 
a string of FGTs connected in series with access transistors to the String Select Line (SSL), the Source line (SL), and the Ground Select Line (GSL), as shown in Figure 2.1. The string length is equal to the total number of FGTs connected in series. The number of pages in a block and the size of each page are integer multiples of the string length, and the number of bit-lines running through the block, respectively. The multiplication factor depends on the architectural layout of the block. Section 2.2.3 explains how the blocks are laid out architecturally. A pass transistor is connected to each word-line to select/unselect the page.

\subsubsection{Basic Flash Operations}

NAND flash uses Fowler-Nordheim (FN) tunneling to move charges to/from the floating gate. A program operation involves tunneling charges to the floating gate while an erase operation involves tunneling charges off the floating gate. Tunneling of charges to and from the FGT varies its threshold voltage and bits are encoded as varying threshold voltage levels. A read operation involves sensing the threshold voltage of the FGT. An erase is performed at the granularity of a block while the read and program operations are performed at a page granularity. Since each FGT in a MLC flash has more than two threshold voltage levels, a read operation for MLC flash involves multiple stages to sense the correct threshold voltage level. An SLC flash has only one stage in a read operation. Most NAND flash memories employ an iterative programming method like Incremental Step Pulse Programming (ISPP) to program an FGT. In iterative programming, a FGT attains its target threshold voltage through a series of small incremental steps. By controlling the duration and the magnitude of program voltage in each step, iterative programming ensures that precise threshold voltage levels are reached. After each iteration, a verify operation is performed to ensure the required threshold voltage level is reached. If not, the operation is repeated until an upper bound on the number of steps is reached. This upper bound is fixed during the design of the chip. If the upper bound is reached and the target threshold voltage level is still not reached, then the program operation fails. As the iteration count increases, the energy required for a program operation also keeps increasing. Section 2.3.5.5 provides more details about how FlashPower estimates program energy consumption using iterative programming. Brewer et al. provide more details on circuit and 


\begin{tabular}{|c|c|c|}
\hline Word-line & $N_{\text {pagesize }}$ cells & $N_{\text {pagesize }}$ cells \\
\hline 1 & Page 0 & Page 1 \\
2 & Page 2 & Page 3 \\
.. &.. &.. \\
.. &.. &.. \\
$N_{\text {page }} / 2$ & $N_{\text {page }}-2$ & $N_{\text {page }}-1$ \\
\hline
\end{tabular}

Table 2.1: Address Map for a SLC Block.

\begin{tabular}{|c|c|c|c|c|}
\hline Word-line & $\begin{array}{c}\text { MSB of first } \\
N_{\text {pagesize }} \\
\text { cells }\end{array}$ & $\begin{array}{c}\text { LSB of first } \\
N_{\text {pagesize }} \\
\text { cells }\end{array}$ & $\begin{array}{c}\text { MSB of last } \\
N_{\text {pagesize }} \\
\text { cells }\end{array}$ & $\begin{array}{c}\text { LSB of last } \\
N_{\text {pagesize }} \\
\text { cells }\end{array}$ \\
\hline 1 & Page 0 & Page 4 & Page 1 & Page 5 \\
2 & Page 2 & Page 8 & Page 3 & Page 9 \\
.. &.. &.. &.. &.. \\
.. &.. &.. &.. &.. \\
$N_{\text {page }} / 4$ & $N_{\text {page }}-6$ & $N_{\text {page }}-2$ & $N_{\text {page }}-5$ & $N_{\text {page }}-1$ \\
\hline
\end{tabular}

Table 2.2: Address Map for a 2-bit MLC Block.

micro-architecture of flash memory [11,90,92].

\subsubsection{Architectural Layout of Flash Memory}

While Figure 2.1 shows the circuit level layout of a flash memory plane, this section explains the page layout in a flash memory block. We illustrate the architectural layout using a SLC and a 2-bit MLC flash as examples, but the layout is similar for all N-bit flash. We note that the architectural layout of SLC and MLC flash can vary even though the underlying circuit layout and implementation are identical for the two. Assuming that there are a total of $N_{\text {pages }}$ in a block and the size of each page is in $N_{\text {pagesize }}$, Tables 2.1 and 2.2, adapted from [102], depict the address map of a flash memory block. In case of SLC flash, each cell stores 1-bit of information and is mapped to a logical page. The time taken to read and program this bit is nearly the same for all pages. In case of 2-bit MLC flash some pages are mapped to the MSB while some other pages are mapped to the LSB. The pages corresponding to LSB are read and programmed faster compared to pages mapped to the MSB. We will refer to pages mapped to the LSB (pages 0,1,2,3, etc. in Table 2.2) as fast pages and pages mapped to the MSB (pages 4, 5, 8, 9, etc. in Table 2.2) as slow pages. 


\subsection{FlashPower: A Detailed Analytical Power Model}

In this section, we derive a detailed analytical power model for NAND flash memory chips. FlashPower uses this derived model to estimate NAND flash memory chip energy dissipation during basic flash operations like read, program and erase and when the chip is idle. Before delving into the details of the model, we list the components that are modeled and the power state machine. We then explain the integration of FlashPower with CACTI [100], followed by the details of the model itself.

\subsubsection{Circuit Components}

With respect to Figure 2.1, the components that dissipate energy are,

- The bit-line (BL) and word-line (WL) wires.

- The SSL, GSL and SL.

- The drain, source and the gate of the SST, GST and PTs.

- The drain, source and control gate of the FGTs.

- The floating gate of the FGTs - Energy dissipated during program and erase operation.

- The Sense amplifiers (SAs) - Energy dissipated during read, program verify and erase verify operation.

In addition to the above components, FlashPower models the energy dissipated by the block and page decoders, and the charge pumps (that provide voltages for read, program, and erase operation). The energy per read, program and erase operation are the sum of the energy dissipated by all the aforementioned components. Since the energy dissipation of I/O pins varies significantly with the design of the circuit board using the flash chip, we do not model their energy. 


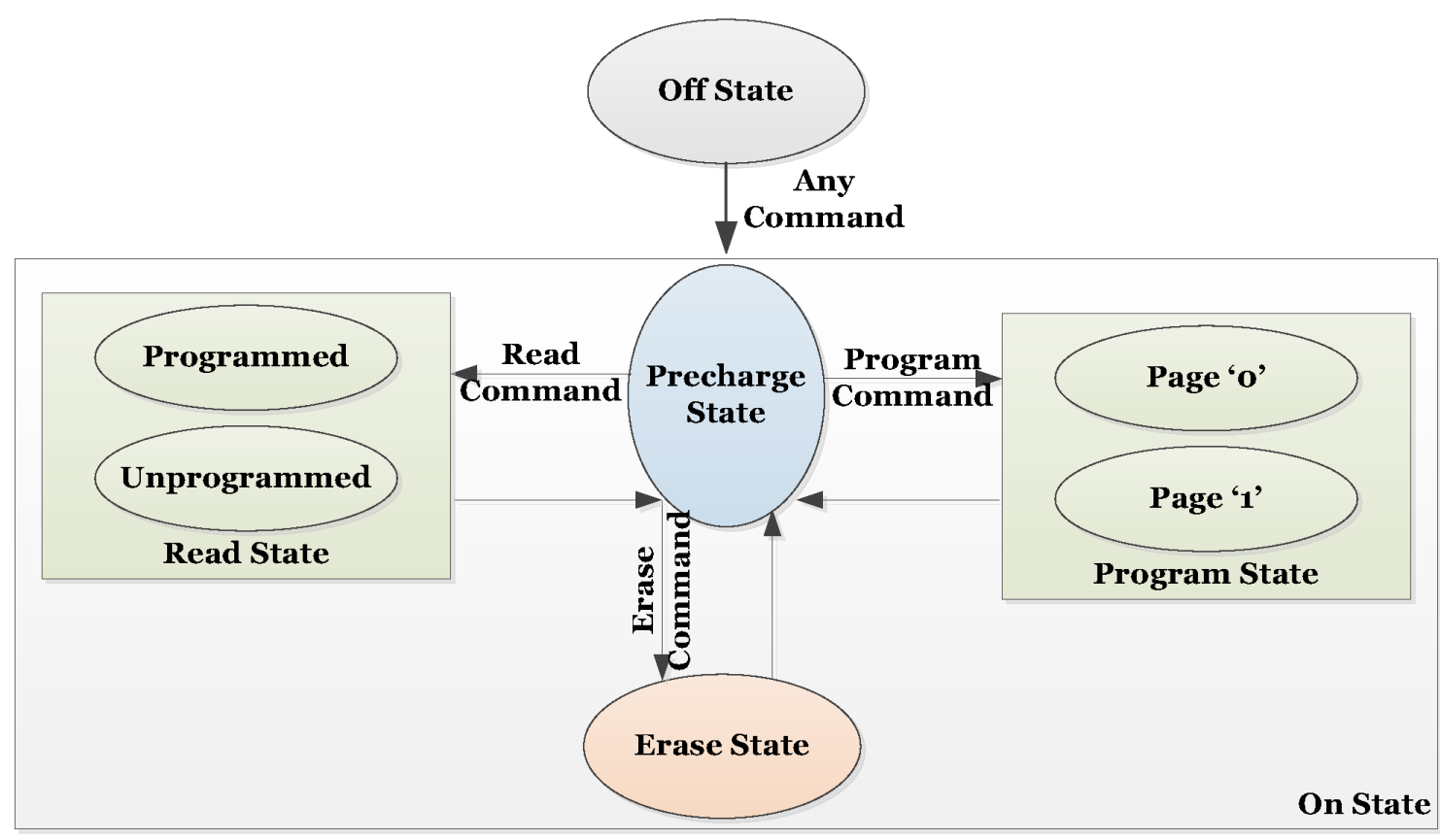

Figure 2.2: Power State Machine for an SLC NAND Flash Chip

\subsubsection{Power State Machine}

Figures 2.2 and 2.3 describes the power state machine for an SLC and an MLC NAND flash chip respectively. The circles represent the individual states, while the solid lines denote state transitions. We model the energy dissipated when the chip is powered. When the chip is on, but is not performing any operations, it is in the "precharge state". In this state, the bit-lines are precharged while the word-lines, and the select lines (SSL, GSL and SL) are grounded. The select lines isolate the array electrically but the chip is ready to respond to commands from the controller.

Upon receiving a read, program, or erase command from the controller, the state machine switches to the corresponding state. When the command is complete, the state machine switches back to the precharge state. We model the energy dissipation of the actual operation and each state transition. For an SLC read operation, depending on whether a page is programmed or not, the chip is in either the "Programmed Page" or the "Unprogrammed Page" state. This can be sensed in only one read cycle. For an SLC program operation, depending on whether the bit to be programmed is a "0" or a " 1 ", the state of the chip is either in "Page 0 " or "Page 1" state.

For a $M L C$ read, the chip transitions to one of the four states, depending on whether it is 


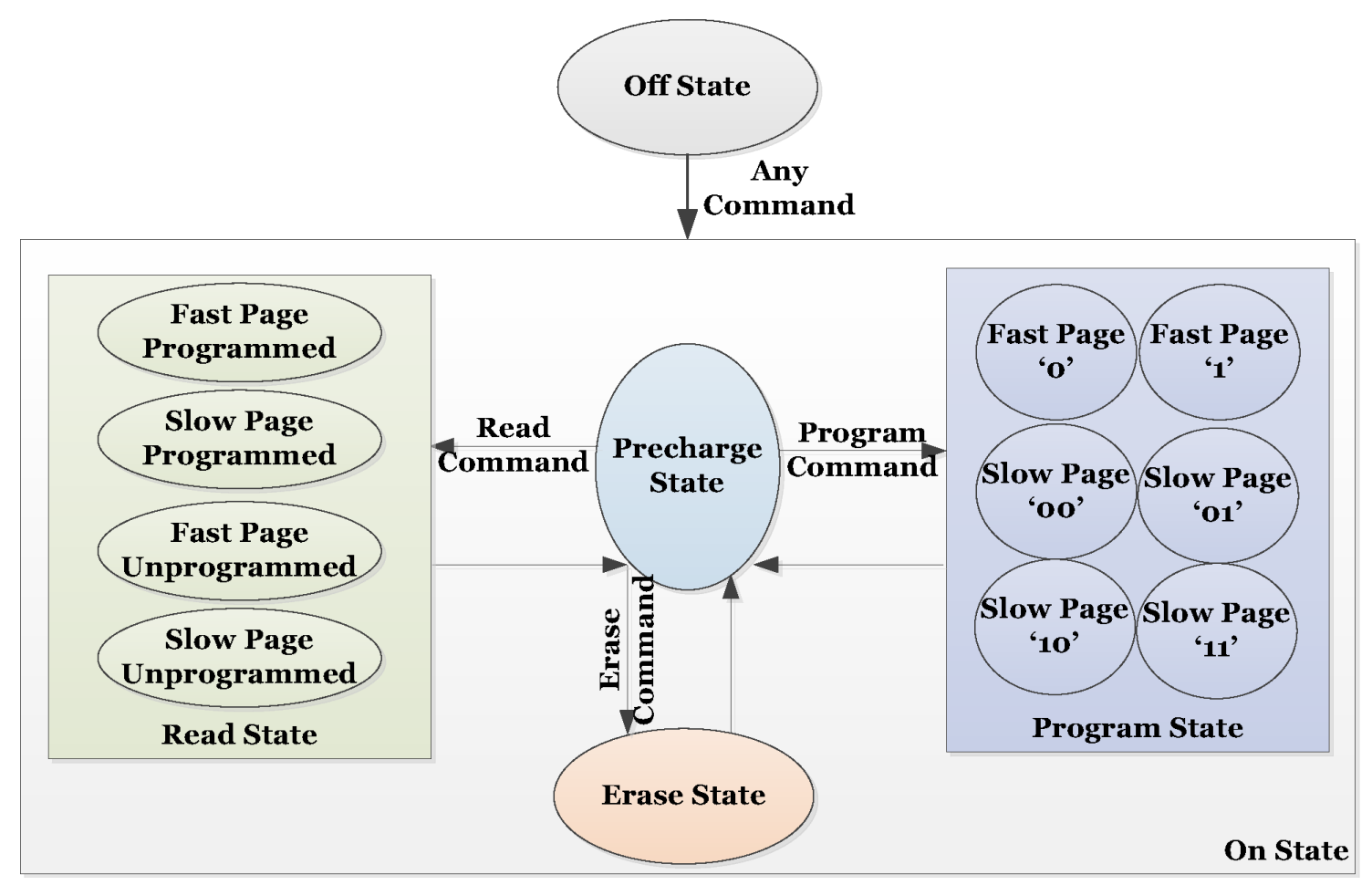

Figure 2.3: Power State Machine for a 2-bit MLC NAND Flash Chip

programmed or not and whether it is a slow or a fast page. For a SLC read, there are only two states based on whether the cell is programmed or not. FlashPower requires atleast 2 read cycles to sense one of the four states for MLC read and two cycles to sense a SLC read.

For an $M L C$ program operation, the state transition of the chip is a function of the bit pattern to be programmed and whether the page is a slow page or a fast page. FlashPower models an conventional multi-page architecture programming method as described in [91]. According to this method, each bit cell stores two pages - a fast page and a slow page. Figure 3 lists all the states for an MLC program while Figure 2.4 explains how the chip transitions between these states. These transitions are based on the multi-page programming algorithm proposed in [91]. When a fast page is programmed, the chip transitions to a "Fast Page 1" state or a "Fast Page 0" state depending on the bit pattern. When a Slow Page is programmed, the chip transitions to one of the four slow page states depending on the bit pattern to be programmed and the current state of the fast page. The main difference between a transition to a "Slow Page 11" state or to a "Slow Page 01" state from a "Fast Page 1" state is the number of steps in the iterative programming method and the threshold 


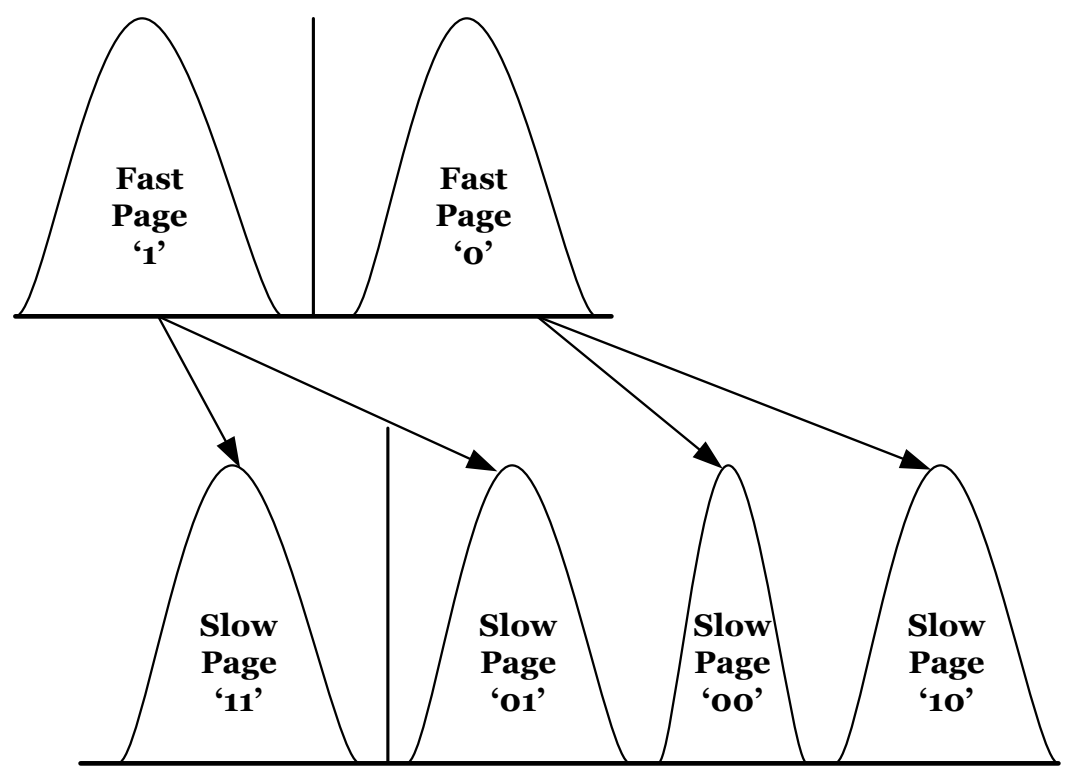

Figure 2.4: Transition between MLC Program States.

voltage difference between the two states. A transition to a slow page state can occur only if the chip is already in one of the two fast page states.

While FlashPower models a canonical MLC program operation, it can also model other programming algorithms as long as they employ iterative programming techniques similar to the multipage architecture model. Small changes to the MLC state machine will be needed to support such algorithms. For example, full sequence programming is a variant of multi-page architecture algorithm in which both both the slow and fast pages are programmed together. To support such an algorithm, the state machine must transition directly from the idle state to one of the four slow page states. Since the main difference between transitions is the number of steps in the iterative programming method and the threshold voltage difference between the states, the programming model does not change, but the values for individual parameters that the model takes will change.

For a $M L C$ program operation, there is only one transition from unprogrammed to programmed state with the unprogrammed state being digitally encoded as " 1 " and the program state encoded as a "0". For an erase operation, we consider only a single state irrespective of whether the block is in SLC or MLC mode. Note that while we consider the bit pattern for program operation, we do not consider the bit pattern for read or erase operation. This is because the read operation only 
involves sensing the threshold voltage of the cell, which is deterministic if all cells in the page are already programmed and is non-deterministic otherwise. For an erase operation, all cells in the block are erased irrespective of their threshold voltage state. In case of the program operation, which involves setting the threshold voltage of the cells to one of the $2^{n}$ states, the input bit pattern makes a significant difference in the power consumption. As we show in Section 2.5, the energy dissipated to reach a "Fast Page 0" state is significantly different from the energy dissipated to reach a "Slow Page 00" state, which in turn is different from the energy dissipated to reach a "Slow Page 01 " state. The important parameters that affect the energy dissipation for the program operation are the threshold voltage difference and the number of verify cycles required to transition to the final threshold voltage state from the start state, both of which are governed by the input bit pattern.

\subsubsection{Extending FlashPower for n-bit MLC flash}

While the state machines in Figures 2.2 and 2.3 are for SLC and 2-bit MLC flash, FlashPower can be extended for other n-bit MLC flash variants like Three Level Cell (TLC) flash. The state machine can be generalized for a n-bit MLC flash as follows: FlashPower has a total of $2^{n}$ states for the read operation and we can sense one of these states within $n$ cycles. Assuming that we employ iterative programming and multi-page architecture similar to 2-bit MLC, the model requires a total of $2^{n+1}-2$ states for the program operation. Since an erase operation is for an entire block irrespective of the threshold voltage of the individual cells in the block, FlashPower has only one state for the erase operation. For TLC flash, this corresponds to 8,14 and 1 states for read, program and erase operation respectively. The number of steps in programming and erase method can vary from one implementation to another and so would the difference in threshold voltage of states between transitions. Since the program and erase model are based on these parameters, they can be extended to support n-bit MLC flash.

\subsubsection{Integration with CACTI}

FlashPower is designed as a plug-in to CACTI [100], a widely used memory modeling tool. We estimate the power consumption of array peripherals such as decoders and sense amplifiers assum- 
ing that they are high performance devices and for the bit-lines and word-lines assuming aggressive interconnect projections. We also model an FGT as a CMOS transistor and then use the CMOS transistor models of CACTI to calculate the parasitic capacitance of an FGT. However, unlike CACTI, which models individual components of SRAM and DRAM based memory systems, we have chosen to model the basic operations on the flash memory. This is because, in the memories that CACTI models, individual operations operate at the same supply voltage to drive the bit-lines and the word-lines. For example in a SRAM-based memory, both read and write operation use the same voltage for word-lines and bit-lines. The granularity (total bytes) of a read/write is also the same. However for NAND flash, read, program and erase operate at different bias conditions and the granularity of an erase differs from read/program. Since the circuitry behaves differently for these different operations, we believe that it is more appropriate to model energy for the basic operations on the flash memory. Table 2.3 summarizes the inputs to FlashPower.

\subsubsection{Power Modeling Methodology}

\subsubsection{Modeling the Parasitics of an FGT}

To calculate the energy dissipation of an FGT, it is necessary to estimate the FGT's parasitic capacitance - i.e. an FGT's source, drain and gate capacitance. While the source and the drain of an FGT and a CMOS transistor are similar, an FGT has a two gate structure (floating and control) while a CMOS transistor has a single gate. We model a dual gate FGT structure as an equivalent single gate CMOS structure by calculating the equivalent capacitance of the two capacitors (one across the inter-poly dielectric and other across the tunnel oxide). We then use CACTI to calculate the parasitics of this transistor. Figure 2.5 illustrates this. We calculate the control gate capacitance $C_{c g, m c}$ using the information on gate coupling ratio (GCR) available in [35], while we calculate the floating gate capacitance $C_{f g, m c}$ using the overlap, fringe and area capacitance of a CMOS transistor of the same feature size. The source and drain capacitance of the transistor depend on whether the transistor is folded or not. FlashPower assumes that the transistor is not folded as the feature size is in the order of tens of nanometers. We use CACTI to model the drain capacitance of the FGT and the parasitic capacitance of other CMOS transistors like the GST, PT and SST. 
Table 2.3: Inputs to FlashPower.

FlashPower has 12 Required(R) and 23 Optional(O) Parameters.

\begin{tabular}{|c|c|}
\hline \multicolumn{2}{|r|}{ Microarchitectural Parameters } \\
\hline$N_{\text {pagesize }}(\mathrm{R})$ & Size (in bytes) for the data area in each page. \\
\hline$N_{\text {sparebytes }}(\mathrm{R})$ & Size (in bytes) for the spare area in each page. \\
\hline$N_{\text {pages }}(\mathrm{R})$ & Number of pages per block. \\
\hline$N_{\text {brows }}(\mathrm{R})$ & Number of rows of blocks in a plane. \\
\hline$N_{b c o l s}(\mathrm{O})$ & Number of columns of blocks in a plane. Defaults to 1 . \\
\hline$N_{\text {planes }}(\mathrm{O})$ & Number of planes per die. Defaults to 1. \\
\hline$N_{\text {dies }}(\mathrm{O})$ & Number of dies per chip. Defaults to 1 . \\
\hline $\operatorname{tech}(\mathrm{R})$ & Feature size of FGTs. \\
\hline Bits_per_cell (R) & Number of bits per FGT. \\
\hline \multicolumn{2}{|r|}{ Device-level Parameters } \\
\hline$N_{A}, N_{D}(\mathrm{O})$ & Doping level of P-well and N-well. Defaults to $10^{15} \mathrm{~cm}^{-} 3$ and $10^{19} \mathrm{~cm}^{-} 3$. \\
\hline$\beta(\mathrm{O})$ & Capacitive coupling between control gate and P-well. Defaults to 0.8 . \\
\hline $\mathrm{GCR}(\mathrm{O})$ & Ratio of control gate to total floating gate capacitance. Obtained from ITRS. \\
\hline$t_{o x}(\mathrm{R})$ & Thickness of tunnel oxide in FGTs. \\
\hline$\frac{W}{L}(\mathrm{O})$ & Aspect ratio of FGTs. Defaults to 1. \\
\hline \multicolumn{2}{|r|}{ Workload Parameters } \\
\hline$N_{\text {bits_1 }}(\mathrm{O})$ & Number of 1's to be read, or programmed. \\
\hline \multicolumn{2}{|r|}{ Timing Parameters } \\
\hline$t_{\text {program }}(\mathrm{R})$ & Latency to program a page for SLC flash (fast page in MLC flash). \\
\hline$t_{\text {program }, \text { slow }}(\mathrm{O})$ & Latency to program a slow page in MLC flash. Defaults to $t_{\text {program }} * 2$. \\
\hline$t_{\text {read }}(\mathrm{R})$ & Latency to read a page in SLC flash (fast page in MLC flash). \\
\hline$t_{\text {read,slow }}(\mathrm{O})$ & Latency to read a slow page in MLC flash. Defaults to $t_{\text {read }} * 2$. \\
\hline$t_{\text {erase }}(\mathrm{R})$ & Latency to erase a flash block. \\
\hline \multicolumn{2}{|r|}{ Bias Parameters } \\
\hline$V_{d d}(\mathrm{R})$ & Maximum operating voltage of the chip. \\
\hline$V_{\text {read }}(\mathrm{O})$ & Read voltage for SLC flash (fast page in MLC flash). Defaults to $4.5 \mathrm{~V}$. \\
\hline$V_{\text {read,slow }}(\mathrm{O})$ & Read voltage for slow page in MLC flash. Defaults to $2.4 \mathrm{~V}$ \\
\hline$V_{b l, d r o p_{-}[0 / 1]}(\mathrm{O})$ & Bitline swing for read operation. Defaults to $0.7 \mathrm{~V}$. \\
\hline$V_{\text {pgm }}(\mathrm{O})$ & Program voltage for selected page. Obtained from ITRS. \\
\hline$V_{\text {pass }}(\mathrm{O})$ & Pass voltage during program for un-selected pages. Defaults to $10 \mathrm{~V}$. \\
\hline$V_{\text {era }}(\mathrm{O})$ & Erase voltage to bias the substrate. Same as $V_{p g m}$ \\
\hline$V_{b l, p r e}(\mathrm{O})$ & Bit-line precharge voltage. Defaults to $3 / 5$ th of $V_{d d}$. \\
\hline$V_{\text {step }}(\mathrm{O})$ & Step voltage used for program and erase. Defaults to $0.3 \mathrm{~V}$ \\
\hline \multicolumn{2}{|r|}{ Policy Parameters } \\
\hline$N_{\text {read_verify_cycles }}(\mathrm{R})$ & Number of read verify cycles for a program operation \\
\hline$N_{\text {erase_cycles }}(\mathrm{R})$ & Number of erase pulses required to erase a block. \\
\hline$\Delta V_{t h, s l c}(O)$ & $\begin{array}{l}\text { Threshold voltage difference between programmed and erased state } \\
\text { for SLC flash. Defaults to } 3 \mathrm{~V} \text {. }\end{array}$ \\
\hline$\Delta V_{t h, m l c}(O)$ & $\begin{array}{l}\text { Threshold voltage difference between adjacent programmed states } \\
\text { for MLC flash. Defaults to } 0.9 \mathrm{~V} \text {. }\end{array}$ \\
\hline Optimize_Write $(O)$ & $\begin{array}{l}\text { Boolean flag enabling optimization to certain threshold levels transitions } \\
\text { in MLC flash. Defaults to false. }\end{array}$ \\
\hline Optimize_Erase $(O)$ & $\begin{array}{l}\text { Boolean flag enabling optimization if the threshold level of a FGT is } \\
\text { unchanged after programming. Defaults to false. }\end{array}$ \\
\hline
\end{tabular}



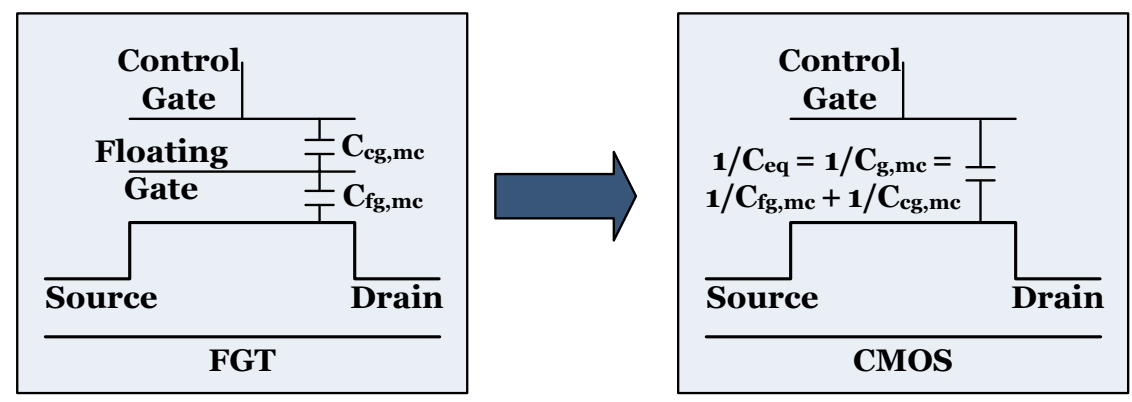

Figure 2.5: Modeling a Floating Gate Transistor

\subsubsection{Derived Parameters}

The length of each bit-line $L_{\text {bitline }}$ and each word-line $L_{\text {wordline }}$ are derived as:

$$
\begin{aligned}
& L_{\text {wordline }}=N_{\text {bitlines-perblock }} * N_{\text {bcols }} * \text { pitch }_{\text {bit-line }} \\
& L_{\text {bitline }}=\left(N_{\text {pages }}+3\right) * N_{\text {brows }} * \text { pitch } \\
& \text { word-line }
\end{aligned}
$$

where $N_{\text {bitlines-perblock }}=\left(N_{\text {pagesize }}+N_{\text {sparebytes }}\right) * 8$. "3" is added to $N_{\text {pages }}$ because for each block, the bit-line crosses 3 select lines (GSL, SSL and SL). The terms pitch bit-line $_{\text {and }}$ pitch word-line $_{\text {refer }}$ to the bit-line and word-line pitch respectively and are equal to $2 *$ featuresize.

The total capacitance to be driven along a word-line is given by:

$$
\begin{gathered}
C_{w l}=C_{d, p t}+C_{g, m c} * N_{\text {bitlines-perblock }} \\
+C_{w l, \text { wire }} * L_{\text {wordline }}
\end{gathered}
$$

where, $C_{d, p t}$ is the drain capacitance of the pass transistor, $C_{g, m c}$ the equivalent gate capacitance of FGT and $C_{w l \text {,wire }}$ is the word-line wire capacitance. Similarly, the total capacitance to be driven along the bit-line is equal to:

$$
C_{b l}=2 * C_{d, s t}+C_{d, m c} * N_{\text {pages }}+C_{b l, \text { wire }} * L_{b i t l i n e}
$$

where $C_{d, s t}$ is the drain capacitance of the select transistors (SST), $C_{d, m c}$ is the drain and source 
capacitance of the FGT and $C_{b l, \text { wire }}$ is the bit-line wire capacitance. Adjacent FGTs connected in series share the source and the drain. Because of this assumption, the source capacitance of one FGT is considered equal to the drain capacitance of the neighboring FGT. The source of SST is shared with the drain of the first FGT in the string while the source of the last FGT in the string is shared with the drain of GST. The total capacitance of the SSL is:

$$
\begin{gathered}
C_{s s l}=C_{g s l}=C_{d, p t}+C_{g, s t} * N_{\text {bitlines }- \text { perblock }} \\
+C_{w l, \text { wire }} * L_{\text {wordline }}
\end{gathered}
$$

Since the GSL needs to drive the same components as the SSL, we have $C_{s s l}=C_{g s l}$. The capacitive component of the SL is equal to:

$$
C_{\text {srcline }}=C_{w l, \text { wire }} * L_{\text {wordline }}+C_{d, s t}
$$

We assume that the source capacitance of GST is equal to its drain capacitance $\left(C_{d, s t}\right)$.

\subsubsection{Transition from the Powered Off to the Precharged State}

When this transition occurs, the bit-lines are precharged and word-lines to $V_{b l, p r e}$ and $V_{w l \text {,pre }}$ respectively. We bias the SST, GST and the PTs so that the current flowing through the FGTs is disabled. We bias the source line to ground. Hence the energy to transition to the precharge state $\left(E_{\text {pre }}\right)$ equals:

$$
\begin{array}{rc}
E_{\text {pre }} & =E_{b l, \text { pre }}+E_{\text {wl }, \text { pre }} \\
E_{\text {bl,pre }}=0.5 * C_{b l, \text { wire }} *\left(0-V_{b l, p r e}\right)^{2} \\
& * N_{\text {bitlines-perblock }} * L_{\text {bitline }} \\
E_{\text {wl,pre }}=0.5 * C_{\text {wl,wire }} *\left(0-V_{\text {wl }, \text { pre }}\right)^{2} \\
* N_{\text {pages }} * L_{\text {wordline }}
\end{array}
$$


While we precharge the bit-lines to reduce the latency of read accesses [48], the word-lines are not.

During our validation we set $V_{w l}$,pre to zero, but the model includes the word-line precharge $V_{w l, p r e}$ as a parameter so that devices that perform word-line precharging can use this parameter.

\subsubsection{The Read Operation}

The read operation for SLC and MLC flash are quite similar. In case of SLC flash and fast pages in 2-bit MLC flash, the sequence of operations required to sense the threshold voltage of the cells is the same since we only need to distinguish between two states. In case of slow MLC pages, we need 2 stages (and hence two read operations) to distinguish between four states.

To perform a read operation for both SLC and MLC flash, the block decoder selects one of the blocks to be read while the page decoder selects one of the $N_{\text {pages }}$ inside a block. For SLC flash and fast pages in MLC flash, we bias the word-line of the selected page to ground while we change the bias of un-selected word-lines to $V_{\text {read }}$ from $V_{w l, p r e}$. This causes the un-selected pages to serve as transfer gates. Hence the energy to bias the word-line of the selected page to ground and the un-selected pages to $V_{\text {read }}$ equals:

$$
\begin{array}{cc}
E_{\text {selected-page, } r}=0.5 * C_{w l} *\left(0-V_{w l, p r e}\right)^{2} \\
E_{\text {unselected-pages }, r}=0.5 * C_{w l} *\left(V_{\text {read }}-V_{w l, p r e}\right)^{2} \\
*\left(N_{\text {pages }}-1\right)
\end{array}
$$

For the read operation, the bit-lines remain at $V_{b l, p r e}$. Depending upon the threshold voltage of the FGT, the FGT is on or off which impacts the voltage drop in the bit-line. Assuming $N_{\text {bits } 1}$ to be the number of bits corresponding to logical " 1 " and $N_{\text {bits }-0}$ to be the number of bits corresponding to logical " 0 ", $V_{b l, d r o p_{-} 1}$ and $V_{b l, d r o p_{-} 0}$ to be the drop in bitline voltages for a 1-read and 0-read, the 
resulting energy dissipation equals:

$$
\begin{aligned}
E_{b l-1, r} & =0.5 * C_{b l} *\left(V_{b l, p r e}-V_{b l, d r o p_{-} 1}\right)^{2} * N_{b i t s_{-} 1} \\
E_{b l-0, r} & =0.5 * C_{b l} *\left(V_{b l, p r e}-V_{b l, d r o p \_}\right)^{2} * N_{b i t s_{-} 0} \\
E_{s l, r} & =E_{s s l, r}+E_{g s l, r}+E_{\text {srcline }, r}
\end{aligned}
$$

where $V_{b l, d r o p_{1} 1}$ is the drop in bitline voltage when FGT is on and $V_{b l, d r o p_{-} 0}$ is the drop in bitline voltage when FGT is off and $E_{s s l, r}, E_{g s l, r}$ and $E_{s r c l i n e, r}$ are given by:

$$
\begin{gathered}
E_{s s l, r}=E_{g s l, r}=0.5 * C_{s s l} *\left(V_{\text {read }}-0\right)^{2} \\
E_{\text {srcline }, r}=0.5 * C_{\text {srcline }} *\left(V_{\text {read }}-0\right)^{2}
\end{gathered}
$$

We detect the state of the cell using the sense amplifier connected to each bit-line. The sense amplifier is a part of the page buffer and contains a latch unit [48]. It detects the voltage changes in the bit-line and compares it with a reference voltage. Since this is very similar to DRAM sense amplifier [99], we use CACTI's DRAM sense amplifier model to determine the energy dissipated for sensing and CACTI's SRAM model to model the energy dissipated to store the sensed data in the page buffer.

In order to read slow pages, we repeat the above steps, but instead of biasing the word-line of the selected page to ground, we bias the word-line of the selected page to $V_{\text {read,slow }}$. If the threshold voltage of the FGT is less than $V_{\text {read,slow }}$, then the FGT is off and the current through the bitline is small. Otherwise, the FGT is on and the current is high. Combining the first and the second read operation helps to distinguish between four threshold stages, thus helping to read the contents of the slow page.

Once the read operation is complete, the system transitions from the read state to the precharged state. This means that we bias bit-lines back to the precharge voltage $V_{b l, p r e}$, while we bias the select lines back to ground from $V_{\text {read }}$ and the word-lines back to $V_{w l, p r e}$. But the biasing for the transition from read operation to precharge state is equal but opposite to the biasing for the transition from 
the precharge state to read operation. Hence the energy to transition from the read to the precharge state $\left(E_{r-p r e}\right)$ equals:

$$
\begin{gathered}
E_{r-p r e}=E_{b l-1, r}+E_{b l-0, r}+E_{\text {selected-page }, r} \\
+E_{\text {unselected-pages }, r}+E_{\text {sl }, r}
\end{gathered}
$$

We calculate the energy dissipated by the decoders for the read operation, $E_{d e c, r}$, using CACTI. We modify the CACTI decoder model to perform two levels of decoding (block and page decode) for each read and program operation. Hence the total energy dissipated per read operation for SLC flash and fast page of MLC flash equals:

$$
\begin{aligned}
E_{r} & =E_{\text {selected-page }, r}+E_{\text {unselected-pages }, r}+E_{b l-1, r} \\
& +E_{b l-0, r}+E_{s l, r}+E_{r-\text { pre }}+E_{\text {senseamp }, r}+E_{\text {dec }, r}
\end{aligned}
$$

The energy for a slow page read operation in MLC flash is equal to:

$$
\begin{gathered}
E_{r, \text { slow }}=E_{r}+E_{\text {selected-page }, r}+E_{\text {unselected-pages }, r} \\
+E_{b l-1, r}+E_{b l-0, r}+E_{s l, r}+E_{r-\text { pre }} \\
+E_{\text {senseamp }, r}+E_{d e c, r}
\end{gathered}
$$

where $E_{\text {selected-page, } r}$ is calculated by biasing the word-line to $V_{\text {read,slow }}$ instead of ground.

\subsubsection{The Program Operation}

To perform a read operation for both SLC and MLC flash, the block decoder selects one of the blocks to be programmed while the page decoder selects one of the $N_{\text {pages }}$ inside a block. We transfer the data from the controller to the page buffers. Since the decoding for the program operation is the same as that of the read operation, we estimate the energy for decoding during the program operation to be equal to $E_{d e c, p}=E_{d e c, r}$, where $E_{d e c, r}$ is the energy for the decode operation estimated 
using CACTI.

FlashPower adopts the incremental step pulse programming model (ISPP) to estimate energy dissipation during program operation [90]. In this paper, we refer to each pulse as a sub-program operation. For each sub-program operation, we increase the program voltage of the selected page by a step factor $V_{\text {step }}$. After each sub-program operation, we perform a verify program operation to check if the correct data is written to the page. If not, we repeat the sub-program operation, but with a higher program voltage. $N_{\text {read_verify_cycles }_{-}}$indicates the total number of sub-program operations performed during the write operation. The duration of each sub-program pulse is a function of the program latency $t_{\text {program }}$ and $N_{\text {read_verify_cycles }}$.

We now calculate the energy for each sub-program operation. Let $V_{p g m, i}$ be the program voltage of the selected word-line in the $i^{\text {th }}$ iteration. We bias the selected word-line to $V_{p g m, i}$ and the unselected word-lines to $V_{\text {pass }}$ to inhibit them from programming. Since the program voltage for the selected page varies for every iteration, we present the energy dissipated by this step as a function of voltage. Thus the energy to bias the selected page and the un-selected page equals:

$$
\begin{aligned}
E_{\text {selected-page }, p}(V) & =0.5 * C_{w l} *\left(V_{\text {pgm }, i}-V_{w l, p r e}\right)^{2} \\
E_{\text {unselected-page }, p} & =0.5 * C_{w l} *\left(V_{\text {pass }}-V_{w l, p r e}\right)^{2} \\
& *\left(N_{\text {pages }}-1\right)
\end{aligned}
$$

FlashPower adopts the the self-boosted program inhibit model [90] to prevent cells corresponding to logical "1" from being programmed. According to the self-boosted program inhibit model, the channel voltage is boosted to about $80 \%$ of the applied control gate voltage by biasing the bit-lines corresponding to logical "1" at $V_{b l, i p}$ and setting the SSL to $V_{d d}$. The resulting electric field across the oxide is not high enough for tunneling and the cells are inhibited from being programmed. Assuming $N_{\text {bits } 1}$ to be the number of bits corresponding to logical "1", the energy dissipated by 
bit-lines to inhibit programming is equal to:

$$
\begin{gathered}
E_{b l, i p}=0.5 *\left(C_{b l}-C_{d, m c} * N_{\text {pages }}\right) \\
* V_{\text {ch_boost }}^{2} * N_{\text {bits_1 } 1}
\end{gathered}
$$

where $V_{\text {ch_boost }}$ is the boosted channel voltage and is a fraction of applied control gate voltage.

We bias the bit-lines corresponding to logical "0" to ground. The resulting high electric field across the tunnel oxide facilitates FN tunneling resulting in charges tunneling from the channel onto the floating gate. The energy dissipation of bitline corresponding to logical " 0 " is equal to:

$$
\begin{aligned}
E_{b l, p} & =\left(0.5 *\left(C_{b l}-C_{d, m c} * N_{\text {pages }}\right) *\left(0-V_{b l, p r e}\right)^{2}\right. \\
& \left.+E_{\text {tunnel }, m c}\right) * N_{\text {bits_o }}
\end{aligned}
$$

where the term $E_{\text {tunnel,mc }}$ is the tunneling energy per cell. $E_{\text {tunnel,mc }}$ is calculated as

$$
E_{\text {tunnel }, m c}=\Delta V_{t h} * I_{F N} * t_{\text {sub-program }}
$$

where $I_{F N}$ is the tunnel current and $t_{s u b-\text { program }}$ is the duration of sub-program operation. $I_{F N}$ is calculated as $I_{F N}=J_{F N} * A_{f g t}$, where $J_{F N}$ is the tunnel current density calculated using [51] and $A_{f g t}$ is the area of the floating gate.

Then the energy dissipated in charging the select lines is equal to:

$$
E_{s l, p}=E_{s s l, p}+E_{g s l, p}+E_{s r c l i n e, p}
$$

where

$$
\begin{gathered}
E_{s s l, p}=E_{g s l, p}=0.5 * C_{s s l} *\left(V_{d d}-0\right)^{2} \\
E_{\text {srcline }, p}=0.5 * C_{\text {srcline }} *\left(V_{d d}-0\right)^{2}
\end{gathered}
$$


Once the sub-program operation completes, NAND flash chips perform a program-verify operation to check if write operation is successful. FlashPower models the verify-program operation as a read operation. Hence, the total energy per sub-program operation is:

$$
\begin{aligned}
E_{\text {subp }}(V) & =E_{\text {selected-page }, p}(V)+E_{\text {unselected-page }, p} \\
& +E_{b l, i p}+E_{b l, p}+E_{s l, p}+E_{v p}
\end{aligned}
$$

where $E_{v p}$, the energy spent in program verification and is given by $E_{v p}=E_{r}-E_{d e c, r} . E_{r}$ is defined by equation (2.18). Since the entire process of sub-program and verify-program is repeated a maximum of $N_{\text {loop }}$ number of times, the maximum energy for programming is given by:

$$
E_{\text {pgm }}=\sum_{i=0}^{N_{\text {read_verify_cycles }}} E_{\text {subp }}\left(V_{\text {pgm, }, i}\right)
$$

$N_{\text {read_verify_cycle }}$ is obtained from datasheets like [77] or can be fed as a input to the model.

Since a program operation concludes with a read operation, the transition from the Program to Precharge is same as the transition from a read to precharge.

$$
E_{p-\text { pre }}=E_{r-\text { pre }}
$$

where $E_{r-p r e}$ is given by equation (2.17).

Hence the total energy dissipated in the program operation is equal to:

$$
E_{p}=E_{d e c, p}+E_{p g m}+E_{p-p r e}
$$

\subsubsection{The Erase Operation}

Since erasure happens at the block granularity, the controller sends the address of the block to be erased. The controller uses only the block decoder and the energy for block decoding $\left(E_{d e c, e}\right)$ is calculated using CACTI. To aid block-level erasing, the blocks are physically laid out such that all pages in a single block share a common P-well. Moreover, multiple blocks share the same 
P-well [90] and therefore it is necessary to prevent other blocks sharing the same P-well from being erased. FlashPower assumes the self-boosted erase inhibit model [90] to inhibit other blocks sharing the same P-well from getting erased.

Once we select an erase block, NAND flash memory uses multiple erase pulses to erase a block. Each such operation is referred to as a sub-erase operation. The bias voltage for the P-well of the selected block, $V_{\text {era }}$, is set to the initial program voltage, $V_{p g m}$. After each sub-erase operation, a read-verify operation determines whether all FGTs in the block are erased or not. If all the FGTs in the block are not erased, we repeat the erase operation (maximum of $N_{\text {erase_cycles }}$ times) after incrementing $V_{\text {era }}$ by the step voltage $V_{\text {step }}$. We denote the erase voltage used for each sub-erase operation to be $V_{\text {sub-erase }, i}$, where $i$ indicates the iteration count of the sub-erase operation. The duration of each sub-erase operation, $t_{\text {sub-erase }}$, is a function of the maximum erase latency and $N_{\text {erase_cycles. }}$.

For each sub-erase operation, we bias the control gates of all the word-lines in the selected block to ground. We bias the P-well for the selected block to erase voltage $V_{\text {sub-erase }, i}$ and the SSL and the GSL to $V_{\text {sub-erase }, i} * \beta$, where $\beta$ is the capacitive coupling ratio of cells between control gate and the P-well. A typical value of $\beta$ is 0.8 [11]. We bias the SL to $V_{b l, e}$. Here $V_{b l, e}$ is equal to $V_{\text {sub-erase }, i}-V_{b i}$ where $V_{b i}$ is the built-in potential between the bitline and the P-well of the cell array [76]. Adding up the charging of the SSL, GSL and SL, we get the energy dissipated in the select lines to be:

$$
E_{s l, e}=E_{s s l, e}+E_{g s l, e}+E_{\text {srcline }, e}
$$

where $E_{s s l, e}, E_{g s l, e}$ and $E_{s r c l i n e, e}$ are calculated using the SSL, GSL and SL capacitance and the bias condition explained above. We bias the bit-lines to $V_{b l, e}$ which dissipates energy that is modeled as:

$$
E_{b l, e}(V)=0.5 * C_{b l} *\left(V_{b l, e}-V_{b l, p r e}\right)^{2} * N_{\text {bitlines-perblock }}
$$

We parameterize $E_{b l, e}(V)$ as a function of applied erase voltage, since the erase voltage changes for each sub-erase operation.

With the P-well biased to $V_{\text {sub-erase }, i}$, cells that have high threshold voltage undergo FN tunnel- 
ing. Electrons tunnel off the floating gate onto the substrate and the threshold voltage of the cell is reduced. To effect FN tunneling, the depletion layer capacitance between the P-well and the N-well should be charged. This capacitance of the junction between the P-well and the $\mathrm{N}$-well, which form a P-N junction, is a function of the applied voltage $V_{\text {era }}$, the area of the p-well $A_{\text {well }}$. The dynamic power to charge this capacitance as the voltage across the junction raises from $0 \mathrm{~V}$ to $V_{\text {sub-erase }, i}$ is determined as:

$$
E_{\text {junction }, e}(V)=\left(\frac{C_{j 0}}{\left(1-V_{\text {sub-erase }, i} / \phi_{0}\right)^{m}} * A_{f g t}\right) * V_{\text {sub-erase }, i}^{2}
$$

where $C_{j 0}$ is the capacitance at zero-bias condition and $\phi_{0}$ is the built-in potential of the P-N junction. $m$, the grading coefficient is assumed to be 0.5 . We parameterize $E_{\text {junction,e }}$ as a function of applied erase voltage since the erase voltage changes for each sub-erase operation.

Once this P-N junction capacitance is fully charged, all cells in the block are erased. The tunneling energy for the block, $E_{\text {tunnelerase } m c}$, is calculated using equation (2.24) and a sub-erase pulse whose duration is $t_{\text {sub-erase }}$. After each sub-erase pulse, a verify erase operation is performed to ensure that all FGTs in the block are erased [90]. The verify erase operation is a single read operation which consumes energy equivalent to a read operation. This is modeled as, $E_{\text {block,ve }}=$ $E_{r}-E_{d e c, r}$ where $E_{r}$ is given by equation (2.18).

Since the erase voltage changes for each sub-erase operation, the total energy consumed in each sub-erase operation, $E_{\text {sub-erase }}$ is parameterized as a function of the voltage and is equal to:

$$
\begin{aligned}
E_{\text {sub-erase }}(V) & =E_{\text {sl }, e}+E_{b l, e}(V)+E_{\text {tunnelerase }, m c}(V) \\
& +E_{\text {junction }, e}(V)+E_{\text {block }, v e}
\end{aligned}
$$

Since an erase operation ends with a read operation, the transition from the erase to precharge is same as the transition from read to precharge. The energy dissipated during this transition is equal to:

$$
E_{e-p r e}=E_{r-p r e}
$$


where $E_{r-p r e}$ is given by equation (2.17).

Hence the total energy dissipated in erase operation $E_{\text {erase }}$ is equal to:

$$
\begin{aligned}
E_{\text {erase }} & =E_{\text {dec }, e}+\sum_{i=0}^{N_{\text {erase_cycles }}} E_{\text {sub-erase }}\left(V_{\text {sub-erase }, i}\right) \\
& +E_{e-\text { pre }}
\end{aligned}
$$

We can observe that if all the cells in the block are programmed and are at a low threshold voltage stage, then an erase operation is not necessary. Some controllers can identify this scenario and prevent an erase operation even when they receive such a command, thereby reducing the power consumption and the latency of the erase operation.

\subsubsection{Charge pumps}

Each read, sub-program, or sub-erase operation requires that the charge pump supply a high voltage (5V-20V) to the flash memory array. Ishida et. al specify that the energy consumed for each high voltage pulse from the charge-pumps is $0.25 \mu \mathrm{J}$ for chips operating at $1.8 \mathrm{~V}$ and $0.15 \mu \mathrm{J}$ for chips operating at 3.3V for the read and program operation [33]. In FlashPower, we use these constant values but since FlashPower is parameterized, a detailed charge-pump model can be incorporated in lieu of the current one.

\subsubsection{Multi-plane operation}

The power model described thus far corresponds to single-plane operations. However modern NAND flash chips allow multiple planes to operate in parallel. These are referred to as multi-plane operations. Since FlashPower models single-plane operations, energy consumption for multi-plane operations can be determined by multiplying the results of single-plane operations with the number of planes operating in parallel.

So far, we have provided an detailed analytical model that estimates the energy dissipation of NAND flash for individual operations. We now validate our model with measurements from real flash chips from various manufacturers. 


\begin{tabular}{|c|c|c|c|c|c|c|c|c|}
\hline $\begin{array}{c}\text { Chip } \\
\text { Name }\end{array}$ & $\begin{array}{c}\text { Feature } \\
\text { Size }(\mathrm{nm})\end{array}$ & $\begin{array}{c}\text { Page Size } \\
(\mathrm{KB})\end{array}$ & $\begin{array}{c}\text { Spare } \\
\text { (bytes) }\end{array}$ & $\begin{array}{c}\text { Pages } \\
\text { /Block }\end{array}$ & $\begin{array}{c}\text { Blocks } \\
\text { /Plane }\end{array}$ & $\begin{array}{c}\text { Planes } \\
\text { /Die }\end{array}$ & $\begin{array}{c}\text { Dies } \\
\text { /Chip }\end{array}$ & $\begin{array}{c}\text { Capacity } \\
(\mathrm{Gb})\end{array}$ \\
\hline B-MLC8 & 72 & $2 \mathrm{~KB}$ & 64 & 128 & 2048 & 2 & 1 & 8.25 \\
D-MLC32 & 80 & $4 \mathrm{~KB}$ & 128 & 128 & 2096 & 2 & 2 & 33.77 \\
E-MLC64 & 51 & $4 \mathrm{~KB}$ & 128 & 128 & 2048 & 4 & 2 & 66.00 \\
A-SLC4 & 73 & $2 \mathrm{~KB}$ & 64 & 64 & 2048 & 2 & 1 & 4.13 \\
B-SLC4 & 72 & 2KB & 64 & 64 & 2048 & 2 & 1 & 4.13 \\
\hline
\end{tabular}

Table 2.4: NAND flash chips used for evaluation

\subsection{Experimental Setup}

To validate the model's usability and accuracy, we measured the latency and energy consumption of read, program, and erase operations from real flash chips. Using a custom-built board, we acquired fine-grain measurements of a diverse set of flash chips. In this section, we describe the hardware setup and the test procedure.

\subsubsection{Hardware Setup}

Figure 2.6 depicts our flash characterization board that consists of two sockets for testing flash chips. The board connects to a Xilinx XUP development kit with a Virtex-II FPGA and an embedded PowerPC processor. The processor runs Linux and connects to a custom flash controller that gives the user complete access to the flash chips. The flash controller can issue operations to the chips and record latency measurements with a $10 \mathrm{~ns}$ resolution.

To measure the energy consumption of individual flash operations, we use a high-bandwidth current probe attached to a mixed-signal oscilloscope. The Agilent 1147A current probe attaches to a jumper that provides power to a single flash chip. We trigger the oscilloscope to capture current measurements when the flash controller sends a command to the flash chip.

\subsubsection{Test Procedure}

Table 2.4 summarizes the system-level properties of the flash chips we used for validation. We selected a diverse set of chips - in terms of feature size, capacity, array structure, and manufacturer - to depict the compatibility and adaptability of FlashPower. 


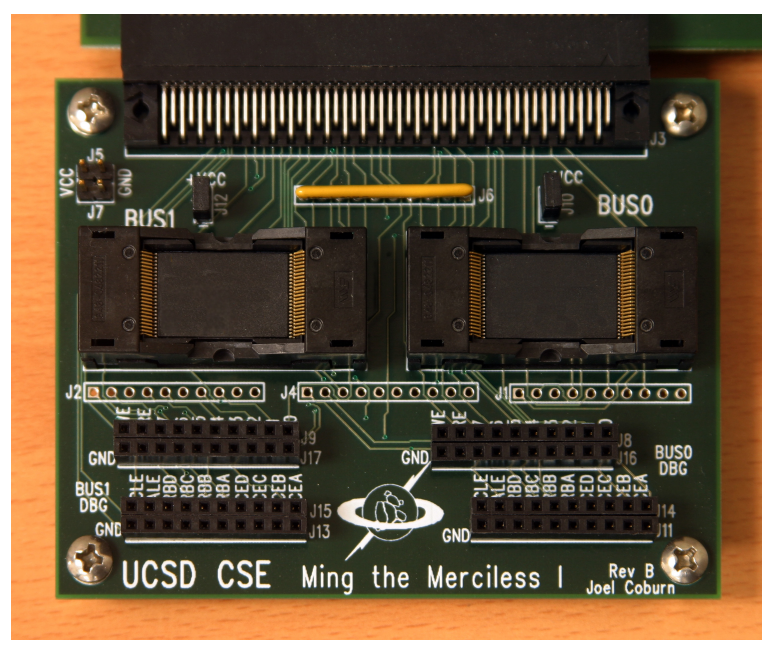

Figure 2.6: Flash Evaluation Board

We measured the latency and power consumption for read, program, and erase operations on each chip. The types of operations we performed varied for SLC- and MLC-based flash chips.

SLC chips We tested four states for a single-page read operation on SLC-based chips: unprogrammed (freshly-erased), all 0's, 50\% 0's, and all 1's. For the program operation, we acquired data while programming a single page with all 0's, 50\% 0's, and all 1's. Finally, for erase operations, we measured the energy while erasing an entire block of pages programmed with all 0's and pages programmed with all 1's.

MLC chips To quantify energy consumption for read operations of MLC-based chips, we measured the energy consumption of reading a page in four states:

1. Fast Page Programmed - Reading a programmed fast page.

2. Slow Page Programmed - Reading a programmed slow page.

3. Fast Page Unprogrammed - Reading a freshly-erased fast page.

4. Slow Page Unprogrammed - Reading a freshly-erased slow page.

For program operations, we measured the energy consumption of the following six transitions caused by programming a page: 
1. Fast Page 0 - Programming a freshly-erased fast page with all 0's.

2. Fast Page 1 - Programming a freshly-erased fast page with all 1's.

3. Slow Page 01 - Programming a slow page with all 1's and the fast page counterpart is of type Fast Page 0.

4. Slow Page 11 - Programming a slow page with all 1's and the fast page counterpart is of type Fast Page 1.

5. Slow Page 00 - Programming a slow page with all 0's and the fast page counterpart is of type Fast Page 0.

6. Slow Page 10 - Programming a slow page with all 0's and the fast page counterpart is of type Fast Page 1.

For erase operations, we measured the energy consumption of erasing an entire block of fullyprogrammed pages of all 0's and all 1's.

For each chip and each type of operation, we performed ten measurements and reported the average. We summarize the results of these experiments in conjunction with the model's approximations in Section 2.5.

\subsection{Validation of FlashPower}

We now compare the outputs of the analytical model with power measurements from real SLC and MLC flash chips. The sources used to obtain values for parameters used in our model (listed in Table 2.3) are as follows: Except for feature size of FGTs, values for all microarchitectural parameters are obtained from manufacturer datasheets. The feature size of FGTs used in our chips are obtained from $[23,83]$. Values for device-level parameters are obtained from $[11,35,76]$. The operating voltage of the chip is obtained from datasheets, while for other bias parameters we use information available in Chapter 6 of [11]. The value for timing parameters and policy parameters are deduced from datasheets, by measuring the latency of flash operations, and from [11]. We used 


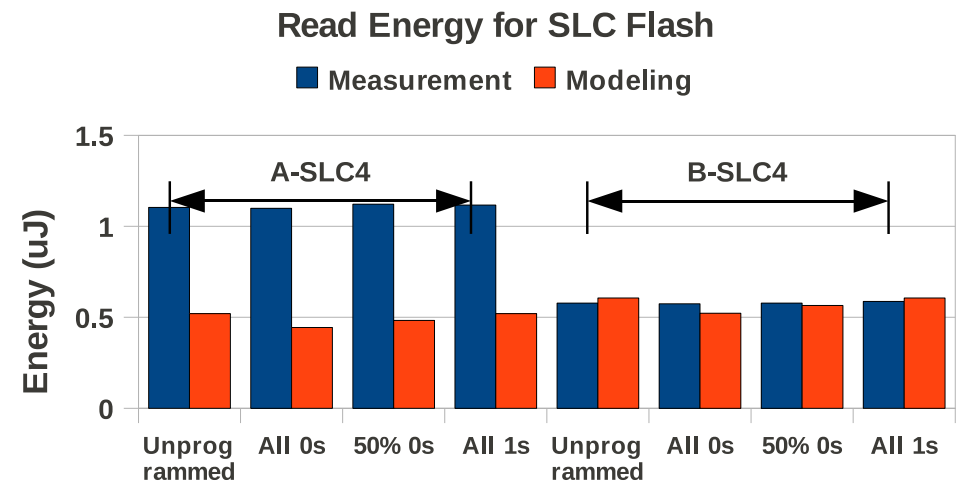

Figure 2.7: Read Energy Comparison for SLC flash

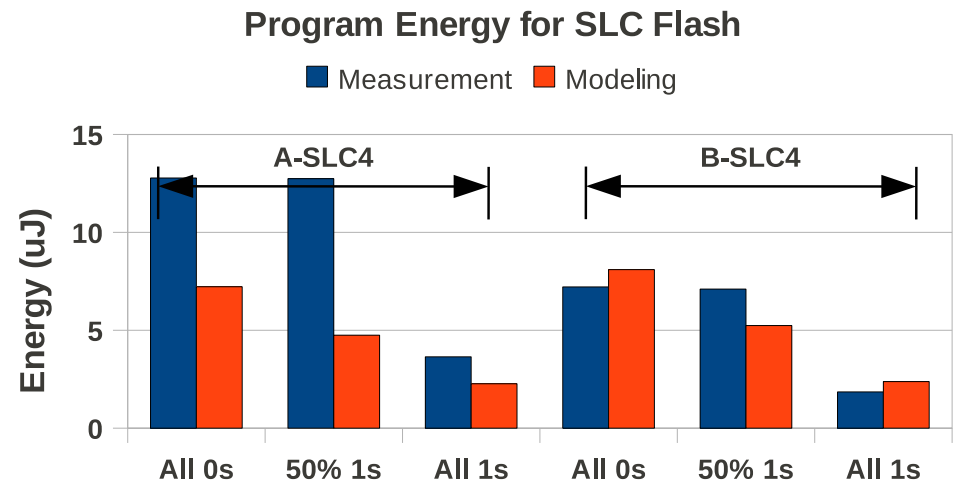

Figure 2.8: Program Energy Comparison for SLC flash

the measured latencies of each flash operation (see Section 4.2) because we found that the values for timing parameters and program verify cycles in datasheets were significantly different (as high as $50 \%$ in some cases) from actual behavior. While datasheets contained average latencies of flash operation, the actual latencies depended on factors like the type of page and in some cases on the data pattern being used. Finally, since we measure the energy consumption of the chips by varying the input bit pattern, we control the values for the workload parameters.

We now present the results for read, program and erase operation for both MLC flash followed by SLC flash. 


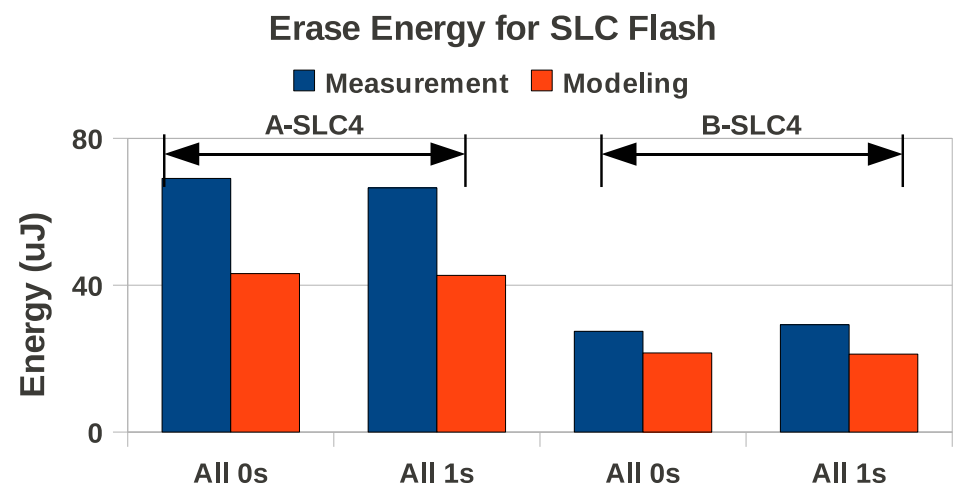

Figure 2.9: Erase Energy Comparison for SLC flash

\subsubsection{Validation for SLC flash}

Figures 2.7, 2.8 and 2.9 show the results for the read, program and erase operations for SLC flash. The y-axis represents the energy consumption of the particular operation, while the $\mathrm{x}$-axis represents the state of a page in SLC flash as explained in Section 2.3.2. From Figure 2.7, we can observe that the variation between the estimated and the measured read energy is about $40 \%$ on average (at most $62 \%$ or $8 \mu \mathrm{J}$ ) for A-SLC4, while it is less than $10 \%$ for B-SLC4. For the program operation, the difference between estimates and measurements is $22 \%$ on average (at most $26 \%$ or $2 \mu \mathrm{J}$ ) for B-SLC4, while the difference is $40 \%$ on average (at most $62 \%$ or $8 \mu \mathrm{J}$ ) for A-SLC4. For the erase operation, it is $24 \%$ on average (at most $27 \%$ or $8 \mu \mathrm{J}$ ) for B-SLC4, while it is $36 \%$ on average (at most $37 \%$ or $24 \mu \mathrm{J}$ ) for A-SLC4.

These results show that the FlashPower can estimate the energy consumption of B-SLC4 with high accuracy, but for A-SLC4, the accuracy is low. It should be noted that the differences among the the measured values of the two chips is similar. In other words, the estimates of A-SLC4 and BSLC4 are comparable among themselves, while the differences in measurements between A-SLC4 and B-SLC4 are high.

To understand why such variations occur, we compare the parameters of the two chips. We find that the microarchitectural parameters for the two chips are almost the same (please refer to Table 2.4). Since the feature size and the operating voltage of the two chips are almost the same, our model assumes that the device level parameters are same. As far as the timing parameters are con- 
cerned, our experiments show that the read latency of A-SLC4 is about $20 \%$ higher than B-SLC4. The program latency for the two chips is similar, while the erase latency is about $30 \%$ higher for ASLC4 compared to B-SLC4. With respect to the policy parameters mentioned in Table 2.4, values for $\Delta V_{t h, s l c}$ is set based on [35] and since the chips have the almost same feature size, their values are same. The values of program-verify and erase cycles are set using latency measurements from the chips and they are similar. Since the bit patterns are constant across both the chips, we hypothesize that the differences in energy are due to changes to specific policy parameters like $\Delta V_{t h, m l c}$ and

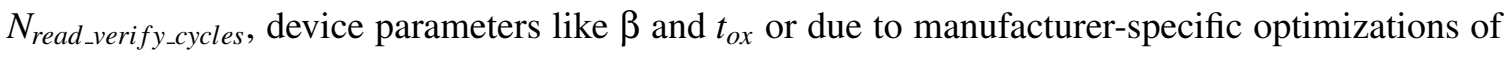
the individual operations. Better knowledge about the values for these parameters can significantly increase the accuracy of the model. FlashPower is developed based on [11, 48, 90, 92] and describes the canonical operation of NAND flash memory and does not capture manufacturer-specific designs. This results in a significant variation between estimates and measurements for A-SLC4 while the variation is significantly lower for B-SLC4. However, since FlashPower is extensively parameterized, any manufacturer-specific implementations can be used for estimating flash power consumption.

\subsubsection{Validation for 2-bit MLC flash}

Figure 2.10 shows the results for the read operation for MLC flash. The $\mathrm{x}$-axis represents the page being read during the operation while the y-axis represents the total energy dissipated during the read. Each column represents the average energy consumed by the flash chip when it is at one of the various states described in Section 2.3.2. From the figure, we can observe that the difference between estimated and measured energy is less than $22.3 \%$ (or $0.64 \mu J$ ) for all types of pages. We found that the difference in estimated and measured energy values were significantly higher for unprogrammed pages than for programmed pages. Since the threshold voltage states of cells in unprogrammed pages is non-deterministic, modeling the energy consumption for an unprogrammed page is challenging. It should be noted that reading an unprogrammed page does not happen often in practice. If we just consider programmed pages, the difference drops to less than $12.3 \%$ (or $0.35 \mu J)$ and in most cases, the difference between measured and estimated values are negligible. 


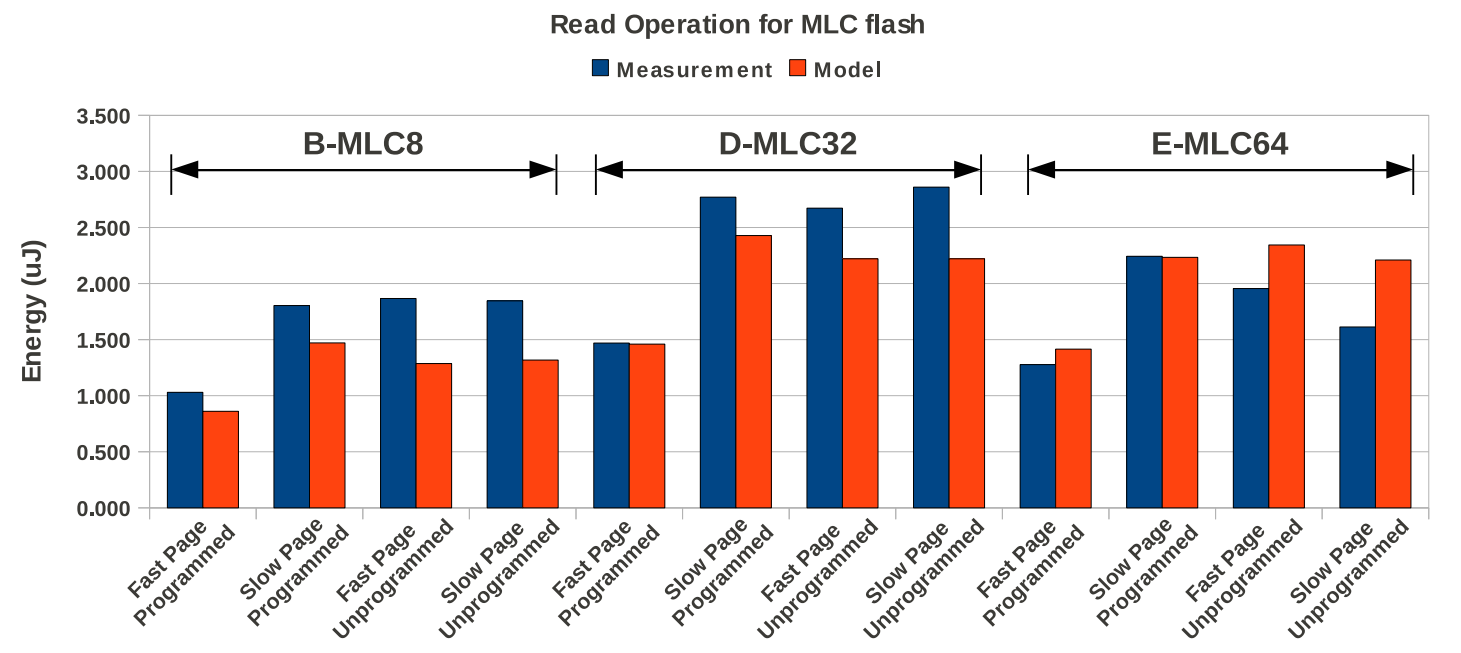

Figure 2.10: Read Energy Comparison for MLC flash

Figure 2.11 shows the result for the program operation for MLC flash. The y-axis represents the energy consumption of a program operation, while the x-axis represents the various states of a flash page. Each column in the figure represents the energy consumed by the flash chip when it is at one of states described in Section 2.3.2. We find that the accuracy of the model is high while programming fast pages for all three flash chips. For slow pages, the accuracy depends on the chip being measured and the bit pattern being used.

Many factors like the number of program verify cycles, the duration of each sub-program operation, the thickness of the tunnel oxide (which affects the tunneling energy), the program and step voltages used, the difference between the starting and the final threshold voltage states affect the energy dissipation during a program operation. Moreover, the values for these factors change with the type of bit pattern being written. Since programming a slow page involves carefully controlling the threshold voltage distribution of the FGT, an accurate value for each of these parameters is even more important. For example, a linear difference in program and step voltage can cause quadratic difference in energy consumption.

While FlashPower identifies values for some factors like number of program-verify cycles used, and the duration of each sub-program operation by reverse engineering the chips, values for $V_{p g m}$, $V_{\text {step }}, \Delta V_{t h, m l c}$ for each chip that is being evaluated are not publicly available. Since values for such 


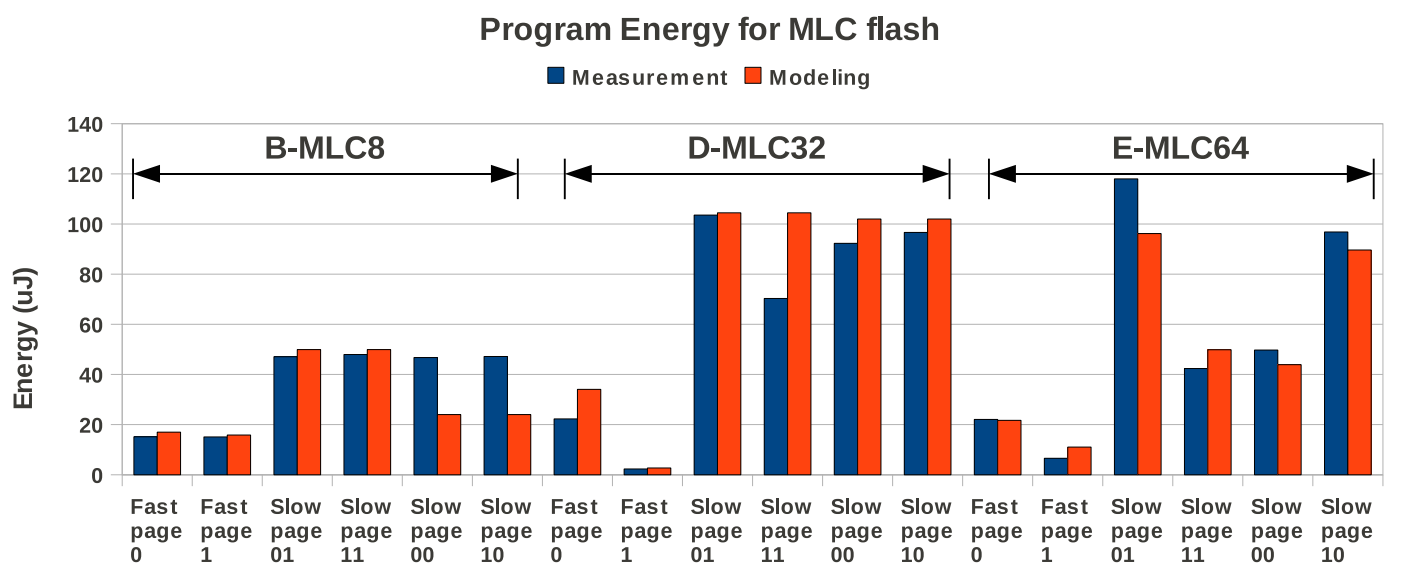

Figure 2.11: Program Energy Comparison for MLC flash

parameters are not publicly available, the variation between estimates and measurements are high for some bit patterns. For example, in the case of B-MLC8, increasing $V_{p g m}$ and $\Delta V_{t h, m l c}$ reduced the difference between the measurement and the estimates for the Slow Page 00 and Slow Page 11 states from $49 \%$ to nearly $8 \%$. In the case of D-MLC32, even a small decrease in $V_{p g m}$ and $\Delta V_{t h, m l c}$ reduces the variation between estimates and measurement for the Slow Page 11 state $49 \%$ to nearly $10 \%$. Since FlashPower has been extensively parameterized, any user who has detailed information on individual parameters can feed those parameters into FlashPower to get an accurate estimate of flash energy consumption.

Figure 2.12 shows the results for the erase operation for the three chips for 2 different bit patterns. For B-MLC8 and D-MLC32, changing the bit pattern did not affect the erase energy significantly. For E-MLC64, when all pages in the block were in the lowest threshold state ("11"), the erase energy was about $5 \mathrm{X}$ lower than the energy consumption for all other bit patterns. We call this the optimize erase operation, where the controller does not erase a block where all cells already have the lowest threshold voltage. While two chips, B-MLC8 and D-MLC32, did not perform this optimization, E-MLC64 had this optimization enabled. From this figure, we can also see that the estimates correspond well to the measurements. The variation between estimates and measurement in less than $10 \%$ for B-MLC8 and E-MLC64, while the difference was less than 20\% for D-MLC32.

So far, we have examined the accuracy of FlashPower by comparing the results with measure- 


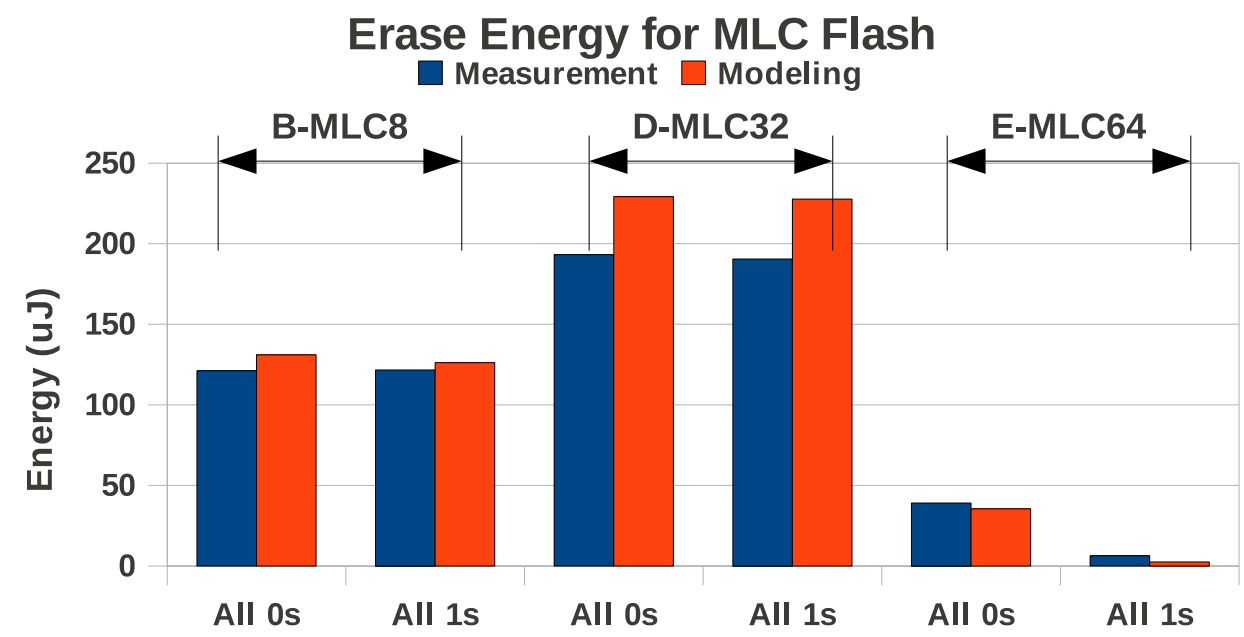

Figure 2.12: Erase Energy Comparison for MLC flash

ments from a range of chips across various manufacturers. We now use the tool to perform some design space exploration studies.

\subsection{Design Space Exploration Using FlashPower}

FlashPower enables us to study the effect of various flash parameters (listed in Table 2.3) on the power consumption of the chip. In this section, we show the utility of FlashPower through two case studies to identify power optimal design configurations.

Case Study 1: Let us say a chip architect decides to design a 16Gb NAND flash chip and wants to estimate the energy consumption for various design scenarios. Table 2.3 provides a list of all parameters that can be adjusted by the architect to identify the relative benefits of each design scenario. Assuming that the designer has the flexibility to change the memory array configuration of flash, we show how to use FlashPower to compare some configurations. For this study, we vary the microarchitecture parameters listed in Table 2.3, except the feature size, while the values for all other parameters are assumed to be the same as the B-MLC 8 chip. Table 2.5 provides a list of configurations for designing a 16Gb memory chip.

Table 2.6 shows the read, write and erase energy for different memory configurations while reading and programming a fast page and erasing a block. Note that, in the case of X3-M16, read 


\begin{tabular}{|c|c|c|c|c|c|c|}
\hline $\begin{array}{c}\text { Chip } \\
\text { Name }\end{array}$ & $\begin{array}{c}\text { Page } \\
\text { Size (KB) }\end{array}$ & $\begin{array}{c}\text { Spare } \\
\text { Bytes }\end{array}$ & $\begin{array}{c}\text { Pages/ } \\
\text { Block }\end{array}$ & $\begin{array}{c}\text { Blocks/ } \\
\text { Plane }\end{array}$ & $\begin{array}{c}\text { Planes/ } \\
\text { Die }\end{array}$ & $\begin{array}{c}\text { Dies/ } \\
\text { Chip }\end{array}$ \\
\hline X1-M16 & 2 & 64 & 128 & 2048 & 2 & 2 \\
X2-M16 & 2 & 64 & 128 & 4096 & 2 & 1 \\
X3-M16 & 4 & 128 & 128 & 2048 & 2 & 1 \\
X4-M16 & 2 & 64 & 256 & 2048 & 2 & 1 \\
X5-M16 & 2 & 64 & 128 & 2048 & 4 & 1 \\
\hline
\end{tabular}

Table 2.5: Different 16Gb Chip Configurations

\begin{tabular}{|c|c|c|c|}
\hline Configuration & $\begin{array}{c}\text { Read Energy } \\
\text { Fast Page } \\
\text { Programmed }(\mu J)\end{array}$ & $\begin{array}{c}\text { Program Energy } \\
\text { Fast Page } \\
(\mu J)\end{array}$ & $\begin{array}{c}\text { Erase Energy } \\
(\mu J)\end{array}$ \\
\hline X1-MLC16 & 1.085 & 15.418 & 117.602 \\
X2-MLC16 & 1.629 & 18.297 & 232.577 \\
X3-MLC16 & 1.632 & 29.747 & 232.908 \\
X4-MLC16 & 1.59 & 18.205 & 227.719 \\
X5-MLC16 & 1.277 & 15.562 & 117.595 \\
\hline
\end{tabular}

Table 2.6: Energy dissipation for various 16Gb Chip Configurations.

and program operations occur at $4 \mathrm{~KB}$ granularity, while for other configurations, they occur at a $2 \mathrm{~KB}$ granularity. The block size is $512 \mathrm{~KB}$ for X3-M16 and X4-M16, while it is $256 \mathrm{~KB}$ for the other configurations. Table 2.6 can be used by the architect to estimate the energy dissipation for various memory configurations. From Table 2.6 we can see that X1-M16 is the chip that dissipates the least energy for read and program operations (for erase operation, the energy dissipation is almost the same as X5-M16). However, the page size of X1-M16 is just 2KB. The page size for X3M16 is $4 \mathrm{~KB}$, while the energy consumption of reads is only $60 \%$ more than X1-M16. Depending upon whether the architect is optimizing for total energy consumption or energy dissipated per byte processed, they can choose one design over the other. In systems that run on battery power and have small data access granularity like consumer electronics devices and wireless sensors, where the architect is limited by maximum energy dissipation of the chip, X1-M16 is better option that X3M16. In systems where energy efficiency is of higher importance than maximum energy dissipation, X3-M16 is a better option. Some designers may choose X5-M16 over the rest because the number of planes in X5-M16 is higher than the rest, which allows for more operations to happen in parallel. Note that for X3-M16, the write and erase energy, is still 2X higher than X1-M16, because their 


\begin{tabular}{|c|c|c|c|}
\hline Configuration & $\begin{array}{c}\text { Read Energy } \\
\text { Fast Page } \\
\text { Programmed }(\mu J)\end{array}$ & $\begin{array}{c}\text { Program Energy } \\
\text { Fast Page } \\
(\mu J)\end{array}$ & $\begin{array}{c}\text { Erase Energy } \\
(\mu J)\end{array}$ \\
\hline All 1s & 1.182 & 8.385 & $2.566 / 115.218$ \\
$75 \% 1 \mathrm{~s}$ & 1.023 & 10.144 & 115.221 \\
$50 \% 1 \mathrm{~s}$ & 0.863 & 11.902 & 115.224 \\
$25 \% 1 \mathrm{~s}$ & 0.703 & 13.660 & 115.226 \\
All 0s & 0.543 & 15.418 & 115.229 \\
\hline
\end{tabular}

Table 2.7: Energy dissipation for various bit patterns for X1-M16.

pages are larger.

Case Study 2: Let us say a chip architect decides to design a NAND flash chip that can consume less energy while storing specific data patterns. This scenario could be motivated by an application behavior or due to flash aware encoding schemes that seek to increase flash lifetime by favoring one bit pattern over an another. For example, a flash aware encoding scheme can choose to write more logical $1 \mathrm{~s}$ than logical $0 \mathrm{~s}$ in order to reduce the probability of tunneling of cells, thereby increasing flash lifetime. FlashPower allows the architect to estimate the impact of energy dissipation due to different bit patterns. Table 2.7 shows the energy dissipation of X1-M16 for read, write and erase operations for various bit patterns. These results are based on the assumption that there is $100 \%$ bitline discharge for logical 1s and no discharge for logical 0s during the read operation. From Table 2.7, we can see that for a read operation, a page filled with 0s consume less energy than a page with 1s because bit-lines corresponding to logical 0 do not discharge while bit-lines corresponding to logical 1 discharges fully and hence dissipates more energy. However, in case of write operations, writing logical 1s consumes less energy because programming is inhibited for logical 1s, while tunneling happens when logical 0s are written, consuming more energy. For erase operations, if the chip supports optimize erase feature, then the erase energy is just $2.5 \mu \mathrm{J}$. Without optimization, this increases by $46 \mathrm{X}$ to $115.128 \mu \mathrm{J}$, indicating the importance of optimizating erase operations. The erase energy does not vary significantly with the data pattern since the entire P-well of the erase block is biased to the erase voltage $V_{\text {era }}$ and hence dissipate almost the same energy.

These two case studies show the utility of FlashPower in evaluating the design tradeoffs for NAND flash memory. Because FlashPower is parameterized, it is possible to analyze such what-if 
scenarios and identify power optimal flash chip configurations.

\subsection{Related Work}

CACTI is an integrated timing, area, leakage and power modeling tool from HP Labs [100]. CACTI supports evaluating SRAM and DRAM memory technologies. This chapter uses the underlying infrastructure available in CACTI and extends it to model NAND flash memories. Research interest in non-volatile memories has spawned development of analytical models for many memory technologies, most of which use CACTI as their base. Smullen et al. provide an analytical model to characterize the behavior of Spin-Transfer Torque RAM (STT-RAM) [86]. Dong et al. provide a similar analytical model for phase change memories [18]. Xu et al. extend CACTI to model Resistive RAM (RRAM) memory technology [101]. While analytical models form one end of the spectrum, characterizing memory technologies with actual measurements form the other end. Grupp et al. provide a characterization of physical properties using actual measurements from flash chips [23]. Yaakobi et al. use results from actual measurements to characterize the reliability of flash chips [102]. Boboila et al. perform a similar study, but focus mainly on write endurance [10]. NVSim presents analytical models for flash with validations at a datasheet level [64]. FlashPower uses measurements from actual chips and combines it with a detailed parameterized analytical model for NAND flash memories. NANDFlashSim is a microarchitecture level tool that simulates only the performance characteristics of NAND flash chips [42]. NANDFlashSim can be used on top of circuit-level analytical modeling tools like FlashPower to provide a flash simulation infrastructure that models both energy and performance.

\subsection{Summary}

In this chapter, we have presented FlashPower, a detailed power model for the two most popular variants of NAND flash namely, the Single-Level Cell (SLC) and 2-bit Multi-Level Cell (MLC) based NAND flash memory chips. FlashPower models the energy dissipation of flash chips from first principles and is highly parameterized to study a large design space of memory organizations. 
We have validated FlashPower against measurements from 5 different chips from different manufacturers and show that our model can accurately estimate the energy dissipation of several real chips. 


\section{Chapter 3}

\section{Modeling NAND Flash Memory Reliability}

\subsection{Introduction}

NAND Flash memory is used in a wide range of systems ranging from consumer electronics devices like Secure Digital (SD) cards and smart phone storage to Solid State Disks (SSDs) in data centers. Due to this wide application, NAND flash based storage systems have diverse reliability requirements. A storage device like a SD card may require data to be stored for several years but may be written only a few hundred times. However, a SSD used in data center may be written several thousand times while the data is expected to remain non-volatile only for a few weeks or months due to the periodic backup operations. In order to understand the reliabilty of NAND based storage systems, a comprehensive understanding of the physical behavior of the memory and various reliability mechanisms along with system parameters (like workload and ambient temperature) used to exercise the storage device is required. In this chapter, we study the major failure mechanisms associated with NAND flash using a detailed analytical model. We then use the analytical model to quantify failure at the memory level and study sensitivity to important parameters like temperature and recovery periods. We then use the model to study the trade-offs among the failure mechanisms at the memory level. In Chapter 4, we use the memory level reliability understanding obtained using these models to study the system level reliability of SSDs used in data centers.

The rest of this chapter is organized as follows: Section 3.2 provides an overview of NAND flash reliability. Section 3.3 describes an analytical model for NAND flash data retention, while 
Section 3.4 analyzes the impact of detrapping on data retention. In Section 3.5, we study the effect of temperature on data retention while we discuss related work in Section 3.6. We summarize this chapter in Section 3.7.

\subsection{Overview of Flash Reliability}

The main types of reliability issues in NAND flash memory are: data retention, write endurance, and disturbs. The duration of time for which the data written in flash memory cells can be read reliably is called the (data) retention period. The number of Program/Erase (P/E) cycles that can be performed on flash cells while guaranteeing a particular retention period determines the (write) endurance. While the retention period and endurance of flash can vary with usage, flash manufacturers guarantee a retention period in the order of years and an endurance of 3000 to 100,000 $\mathrm{P} / \mathrm{E}$ cycles $[34,72]$. Loss of data retention and write endurance are the most dominant flash reliability Memory cell disturbs occur when a bit flips inadvertently in that cell due to accesses to adjacent cells. There can be read, write, or erase disturbs, depending on the operation in the adjacent cells. Many studies have shown that NAND flash memory has very low vulnerability to disturbs and that erasing the cell (which occurs several times as part of the normal usage of the SSD, since NAND flash does not support in-place writes) reduces the susceptibility of memory cells to bit flips $[14,93]$. Moreover, existing Error Correction Codes (ECC) in flash memory are effective in handling errors that occur due to disturbs [14]. Therefore this paper focuses only on endurance and retention time and the tradeoffs between the two. Memory standards organizations, such as Joint Electron Device Engineering Council (JEDEC), provide detailed information about these these failure mechanisms [40].

\subsubsection{Factors affecting Flash Reliability}

While a comprehensive analysis of flash memory requires examining all the components in a flash chip, this work only focuses on the flash memory array, which occupies nearly $80 \%$ of the total 
chip area and is the most important component of a flash chip [11]. Some key factors affecting flash reliability are: (1) Cycling, (2) Recovery period, and (3) Temperature.

\subsubsection{Cycling}

FN tunneling requires very high electric fields which affects the reliability of the tunnel oxide in flash [51]. Consequently, program and erase operations, which use FN tunneling, are also referred to as stress events [60]. Cycling breaks the atomic bonds in the oxide layer, which increases the probability of charges getting trapped when they tunnel through the oxide layer. When charges are trapped in the tunnel oxide, it increases charge leakage from the floating gate due to a process called Trap Assisted Tunneling (TAT) [57]. This leakage current is called as Stress Induced Leakage Current (SILC) and is exacerbated due to trap assisted tunneling under low electric fields.

As cycling on the flash memory cell increases over a period of time, charge trapping in the tunnel oxide also increases, which increases SILC. As SILC increases, the data retention period and endurance of flash also decreases.

\subsubsection{Recovery Period}

Flash cells experience a period of relative inactivity before they are chosen to be programmed or erased again. This time period between successive stress events is called as the recovery period because flash memory cells experience some recovery from the effect of stresses during this time period [60]. Prior work $[55,93,103]$ has shown that as the recovery period increases, some of the charges trapped in the tunnel oxide detrap and thereby increase the strength of the tunnel oxide. A recent study [60] provides a detailed analytical model that examines the effect of cycling and recovery periods on trapping and detrapping and their impact on flash endurance. It is important to note that while detrapping of charges from the tunnel oxide helps to keep the oxide clean, it also results in an increase in bit-errors. This is because the read circuit inside flash chips cannot differentiate between charges in the floating gate and the tunnel oxide and charges draining out from the tunnel oxide or from the floating gate are just perceived as shifts in threshold voltage. 


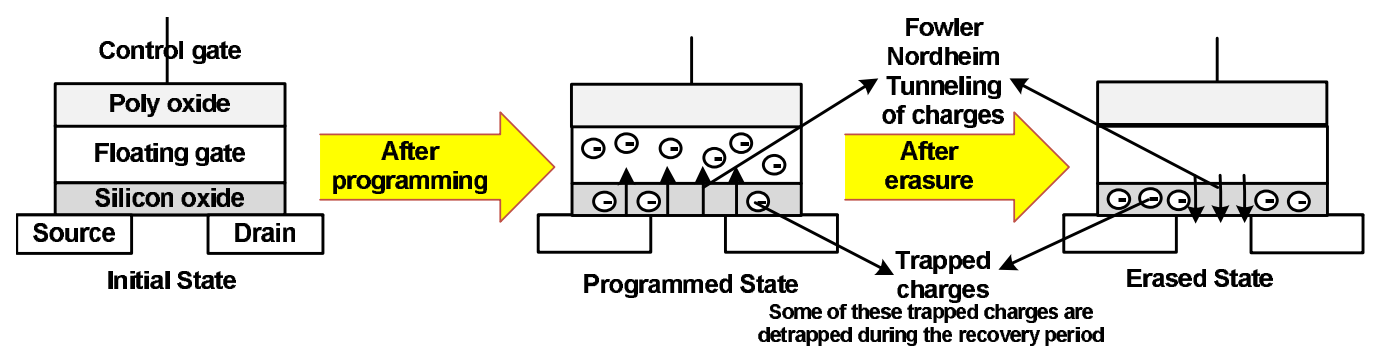

Figure 3.1: Trapping and Detrapping in Floating Gate Transistors

An illustration of trapping during program or erase cycles and of detrapping during recovery periods is shown in Figure 3.1.

\subsubsection{Temperature}

High temperatures can exacerbate several silicon reliability problems and flash memory is no exception. The impact of temperature on the reliability of flash is typically expressed using the Arrhenius equation, where, given a failure rate at stress temperature $T_{\text {stress }}$, the failure rate at another temperature $T_{\text {use }}$ can be calculated by measuring the acceleration factor $(A F)$, which is given by:

$$
\text { AccelerationFactor }(A F)=\exp ^{\frac{E_{a}}{K} \cdot\left(\frac{1}{T_{\text {use }}}-\frac{1}{T_{\text {stress }}}\right)}
$$

where $E_{a}$ is the activation energy of a given type of failure mechanism which is usually determined based on empirical measurements, $K$ is the Boltzmann's constant, $T_{u s e}$ is the temperature in operating conditions (in kelvins) and $T_{\text {stress }}$ is the temperature under stress conditions (in kelvins). JEDEC indicates an activation energy of $1.1 \mathrm{eV}$ for detrapping, while the activation energy for the data retention failure mechanism is indicated to be $0.3 \mathrm{eV} \mathrm{[40].} \mathrm{As} \mathrm{the} \mathrm{activation} \mathrm{energy} \mathrm{increases,}$ the sensitivity of the failure mechanism to temperature significantly increases.

In order to understand how the various factors mentioned in this section affect flash reliability, we now present an analytical model. 


\subsection{An Analytical Model for NAND Flash Data Retention}

We estimate the retention period by modeling the SILC due to charge trapping and detrapping and use this estimate to calculate the duration of time before data loss occurs. By estimating the retention period of flash and the number of $\mathrm{P} / \mathrm{E}$ cycles it takes for the retention to drop below a specific threshold, we estimate the endurance of flash memory. This analytical model is developed by combining information from device physics papers on NAND flash memory cells $[47,55,56$, 103,104]. These papers provide information about the various parameters affecting endurance and retention, their relationship to each other, and values for some fitting constants that are used in the model. It is important to note that all these device physics papers are based on very similar flash memory technology and hence are consistent with each other.

In order to estimate SILC, data retention, and endurance, we need to first determine the effect of cycling and recovery on trapping and detrapping. We use the analytical model developed by Mohan et al. to capture this relationship [60]. They show that the threshold voltage shift due to trapped charges in tunnel oxide has a power law relationship with cycling while recovery period has a logarithmic effect on detrapping. They also show the relationship between cycling and trapping to be:

$$
\delta V_{t h, s}=\frac{\left(A \cdot c y c l e^{0.62}+B \cdot c y c l e^{0.30}\right) \cdot q}{C_{o x}}
$$

where $A$ and $B$ are constants, cycle is the number of P/E cycles, $q$ is the charge of an electron, and $C_{o x}$ is the capacitance of the oxide. Mohan et al. show the relationship between detrapping and recovery period to be:

$$
\delta V_{t h, r}= \begin{cases}\delta V_{t h, p r} & \text { if } \delta V_{t h, p r}<\mathrm{K} * \delta V_{t h, s} \\ K * \delta V_{t h, s} & \text { otherwise }\end{cases}
$$

In the next section, we use Equations 3.2 and 3.3 to develop a reliability model to estimate the effect of trapping and detrapping on the data retention of flash. 


\subsubsection{Model for Data Retention}

The retention model estimates the duration of time taken by the memory cell to leak the charges stored in the floating gate. To determine this time duration, the model estimates SILC based on the number of charges trapped in oxide layer which is a function of the total number of stress events and recovery periods.

According to de Blauwe et al., SILC $\left(J_{S I L C}\right)$ is a sum of two components, (a) a time-dependent transient component $\left(J_{t r}(t)\right)$ and (b) a time-independent steady state component $\left(J_{s s}\right)[37,38]$. We have,

$$
J_{S I L C}=J_{t r}(t)+J_{s s}
$$

Moazzami et al. observed that for thinner tunnel oxides $(<13 \mathrm{~nm})$, the steady state component dominates the transient component [57]. As DiMaria et al. show this steady state component predominantly originates from a trap-assisted tunneling mechanism, where presence of interface and bulk traps increase the leakage current [17]. Hence, in order to obtain a first order reliability model of $J_{S I L C}$, it is sufficient to model the time independent steady state component, $J_{S S}$. So, Equation (3.4) can be modified to

$$
J_{S I L C} \approx J_{S S}
$$

Because the tunnel oxide thickness is smaller than $13 \mathrm{~nm}$ for modern NAND flash generations [34], Equation (3.5) provides a good estimate of SILC. According to Larcher et al., the steady state component is modeled by using a Fowler-Nordheim (FN) like expression, as given below [47].

$$
\begin{aligned}
J_{S I L C}=J_{S S} & \left.=A_{S I L C} \cdot F_{O X}^{2} \cdot \exp ^{(}-\frac{B_{S I L C}}{F_{O X}}\right) \\
A_{S I L C} & =C \cdot J_{S T R}^{\beta} \cdot \exp ^{\left(-D \cdot Q_{I N J}^{\alpha}\right)}
\end{aligned}
$$

The barrier height used to calculate the exponential factor $B_{S I L C}$ is between $0.8-1.1 \mathrm{eV}$. $F_{O X}$ 
is the electric field across the tunnel oxide during stress events and is considered to be $3.8 \mathrm{MV} / \mathrm{cm}$. The values for constants $C, \beta, D, \alpha$ are available in [47]. The term $J_{S T R}$ represents the current density across the tunnel oxide during stress events and is a function of applied program or erase voltage. We assume an operating voltage of $16 \mathrm{~V}$ for program and erase operations based on data from the ITRS Roadmap [34]. $Q_{I N J}$ is the total amount of charge exchanged across the tunnel oxide and is a function of $\mathrm{P} / \mathrm{E}$ cycles. Incorporating the cycling term in $Q_{I N J}$ helps to calculate the leakage current as a device is cycled over a period of time. Based on [47], $Q_{I N J}$ can be defined as,

$$
\begin{aligned}
Q_{I N J} & =\Delta Q_{I N J} \cdot N_{C} \\
\Delta Q_{I N J} & =\Delta V_{t h} \cdot C_{C G}
\end{aligned}
$$

where $\Delta V_{t h}$ is the threshold voltage difference between programmed and erased state, $N_{C}$ is the $\mathrm{P} / \mathrm{E}$ cycle count, and $C_{C G}$ is the capacitance between the control gate and floating gate of a FGT.

Equation (3.6) represents the SILC due to the presence of trapped charges in the tunnel oxide. Because $J_{S I L C}$ is dominated by $J_{S S}$ and $J_{s S}$ remains constant with time [38], the leakage current $\left(J_{S I L C}\right)$ can approximately be considered to be constant with time. Assuming that $\delta Q_{t h}$ to be the total charge stored in the floating gate corresponding to a logical bit, $\delta V_{t h}$ to be threshold voltage shift due to charge trapping (calculated from Equations 3.2 and 3.3), and $J_{S I L C}$ to be the leakage current, we can calculate the time taken for the charges to leak from the floating gate $\left(t_{\text {retention }}\right)$ to be,

$$
t_{\text {retention }}=\frac{\left(\delta Q_{t h}-\left(\delta V_{t h} \cdot C_{C G}\right)\right)}{J_{S I L C}}
$$

After $t_{\text {retention }}$ seconds, most of the charges from the floating gate would have leaked through the tunnel oxide and any read operation has a high probability of returning incorrect data. When this happens, we consider the memory cell to have reached its retention limit and the number of $\mathrm{P} / \mathrm{E}$ cycles it takes for the cell to reach its retention limit is defined as the endurance limit of the cell. Since $\delta V_{t h}$ and $J_{S I L C}$ are functions of stress events and recovery period, Equation (3.10) provides 
an estimate of data retention period in NAND flash memory after taking stress and recovery into account.

\subsection{Impact of Detrapping on Data Retention}

Using the analytical model that we developed in the previous section, we analyze the impact of recovery and detrapping on data retention. We examine when data retention related failures occur and how recovery periods help in increasing data retention of flash. The results of this analysis are shown in Figure 3.2a for SLC and Figure 3.2b for 2-bit MLC flash. These results use a flash feature size of $80 \mathrm{~nm}$ because the cell-level reliability measurements are available only for this technology node. These estimates are based on an operating temperature of $30^{\circ} \mathrm{C}$, the typical ambient temperature of the storage system in datacenters [69].

Typically, OEM datasheets specify a retention period of at least 10 years for NAND flash. This rating is usually very conservative and many supplementary documents provided by OEMs show that the typical retention period is around 100 years for NAND flash memory that has undergone very little cycling $[62,63]$. Our results show that the data retention period of SLC based flash is about 65 years (nearly 23 years for 2-bit MLC flash) for relatively small P/E cycle counts, which is similar to datasheet estimates.

From Figures 3.2a and 3.2b, we can observe that, when there is no recovery between successive cycles, the memory cell encounters retention failure when the total number of P/E cycles is around 100K for SLC flash and 10K for MLC flash, which concurs with the conservative values specified in the datasheets. However, as the recovery period between successive stress events increases, the number of times the cell can be cycled before retention failure occurs increases exponentially. Both SLC and 2-bit MLC flash experience a steep drop in their retention period when they are subjected to a few hundreds to thousands of cycles. For SLC flash, the retention period drops from about 65 years (for no recovery period) to about 20 years in a few hundred cycles, while in case of MLC flash, the retention period drops from nearly 23 years (for no recovery period) to around 5 years in a few thousand cycles. However, after this steep drop, the rate of decrease in the retention period slows 


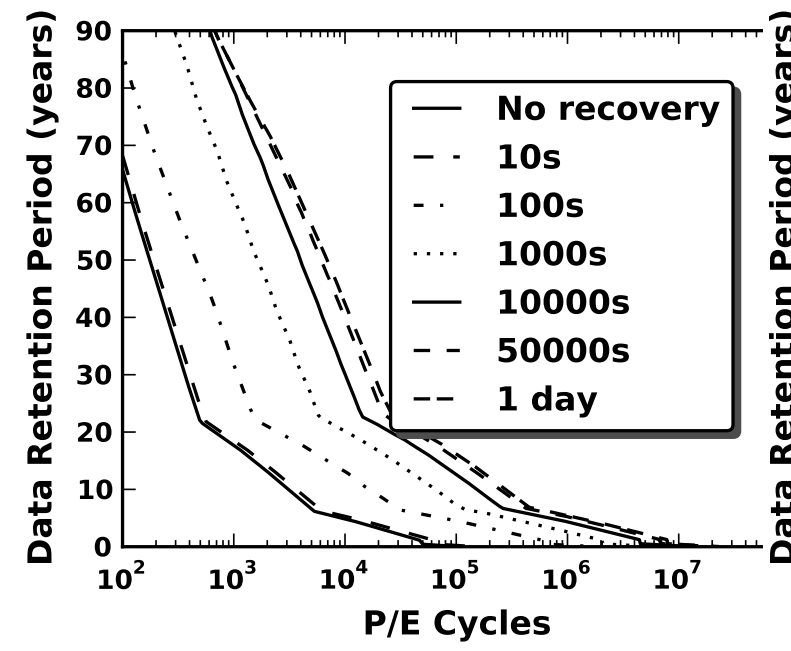

(a) SLC flash

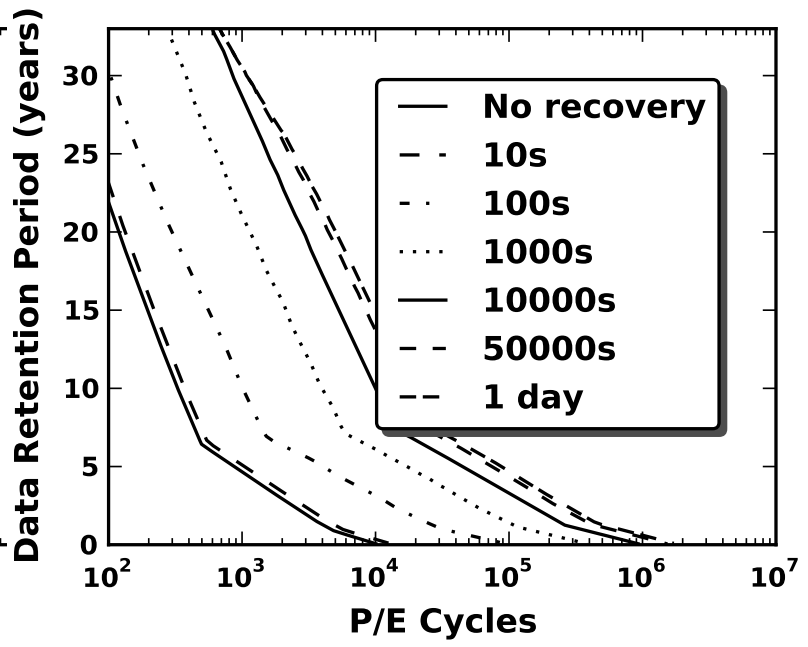

(b) 2-bit MLC flash

Figure 3.2: Impact of different recovery periods on the Data Retention for 80nm SLC and 2-bit MLC flash at $30^{\circ} \mathrm{C}$

down. As the memory cell is cycled, its retention period keeps dropping and when the memory cell is cycled beyond a certain threshold (the write endurance limit), the flash memory cell experiences retention failure, the point at at which the data stored in the memory cell is lost. From Figure 3.2a and Figure 3.2b, we can note that even though SLC flash and 2-bit MLC flash exhibit the same trend, the initial retention period of a new MLC flash memory is significantly lower than that of SLC memory. This is because, the $\Delta V_{t h}$ for MLC flash is about 2.6 times lower than the $\Delta V_{t h}$ for SLC flash.

Another distinct trend that we can observe is that the slope of the curves vary with the amount of cycling the cell has experienced. This behavior is in accordance with the study by Larcher et al. [47]. They show that as cycling increases, the dominance of parameters affecting leakage also varies and hence the rate of change of leakage also varies [47]. For P/E cycles less than $10^{3}$, the drop in threshold voltage is dominated by $A_{S I L C}$, while for P/E cycles greater than $10^{5}$, the threshold voltage drop saturates because the oxide field across the tunnel oxide $\left(F_{o x}\right)$ decreases and the leakage current also decreases. Between $10^{3}$ and $10^{4}$ cycles, both $A_{S I L C}$ and $F_{o x}$ interact with each other creating a different slope. Because the slope of the curve changes, the rate of change in 


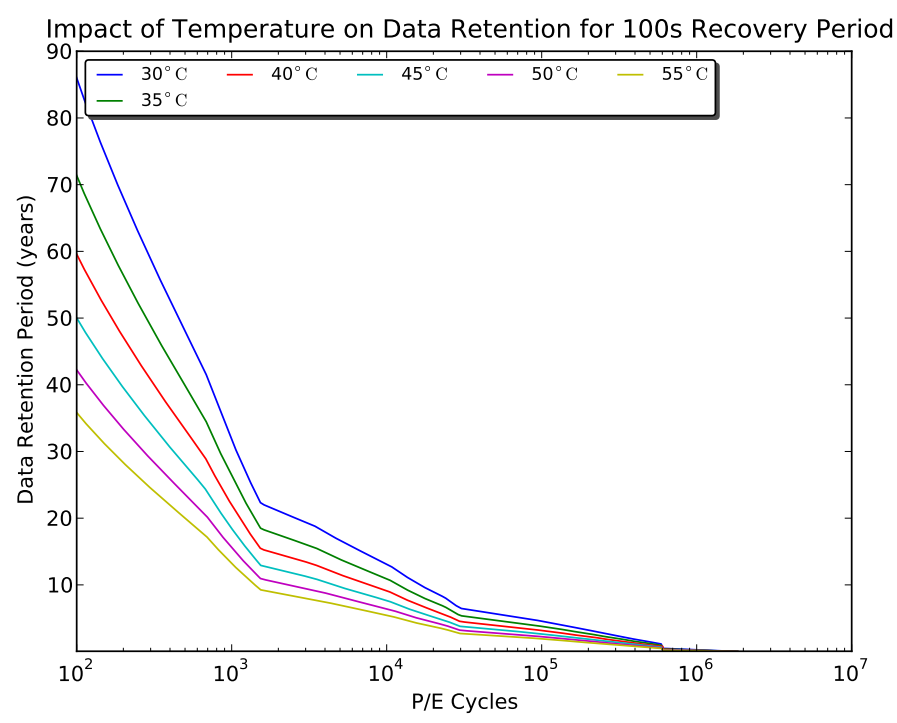

Figure 3.3: Impact of temperature on data retention for SLC flash with 100 seconds recovery period.

retention period also varies significantly with cycling.

\subsection{Impact of Temperature on Data Retention}

We use the Arrhenius equation to study the effect of temperature on flash reliability. As our objective is to study the reliability of SSDs in data center environment, we choose a variety of temperatures ranging from $30^{\circ} \mathrm{C}$ to $55^{\circ} \mathrm{C}$, which is the typical operating temperature range in data centers. The temperature ranges are representative of the typical operating temperature for disks in data centers $[69,82]$. The baseline results were obtained by setting $T_{\text {use }}$ to $30^{\circ} \mathrm{C}$, which is a typical ambient temperature at which disks operate in data centers.

Based on industry standards like JEDEC, the activation energy for data retention is considered to be $0.3 \mathrm{eV}$ [40]. Figure 3.3 and Figure 3.4 illustrate the change in retention period as temperature increases from $30^{\circ} \mathrm{C}$ to $55^{\circ} \mathrm{C}$. while the recovery period is kept constant at 100 seconds. With the data retention activation energy set at $0.3 \mathrm{eV}$ and the detrapping activation energy set at $1.1 \mathrm{eV}$, when the operating temperature increases from $30^{\circ} \mathrm{C}$ to $35^{\circ} \mathrm{C}$ the time to failure (the data retention period) reduces by $18 \%$ causing the chip to become less reliable. Thus temperature has a major impact on 


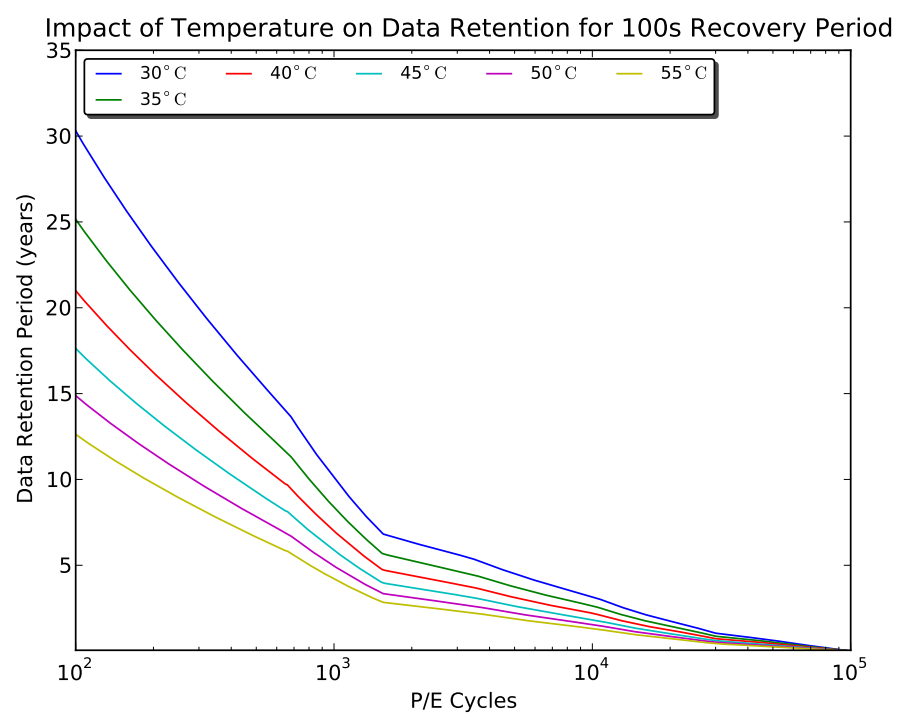

Figure 3.4: Impact of temperature on data retention for 2-bit MLC flash with 100 seconds recovery period.

the reliability of flash memory. These results can be used to estimate how flash memory operates at various temperature points and the expected endurance and retention at these temperatures.

It should be noted that even though charge detrapping increases with temperature (due to higher activation energy), temperature has a negative effect on the overall reliability of flash. Proposal like [71] try to exploit detrapping at higher temperature by heating the SSDs to high temperatures. As our results show, the problem with this approach is that while such techniques increase detrapping, they also increase SILC, resulting in reduced retention period. Because temperature is a important parameter that affects silicon and SSD reliability, both SSD architects and system administrators have a critical role to play. The cooling choices adopted by system administrators to control the ambient temperature of servers can have a significant impact on the reliability of SSDs. Similarly, SSD architects can influence the reliability of SSDs by placing temperature sensors inside SSDs that can be used to monitor and control their reliability.

Figures $3.2 \mathrm{a}$ and $3.2 \mathrm{~b}$ show that there is clear tradeoff between the data retention and the endurance of flash memory. If the data retention for NAND flash can be relaxed linearly, the total number of $\mathrm{P} / \mathrm{E}$ cycles possible can be increased exponentially. For example, in the case of 2-bit 
MLC flash having an average recovery period of about 10,000 seconds, the P/E cycle count can be increased from 10000 to 52,800 when the data retention period requirement is be reduced from 10 years to 5 years.

These results indicate that there is a tradeoff between endurance and data retention for both SLC and MLC flash that can be exploited by storage systems to optimize either of these metrics. In Chapter 4, we show how to tradeoff of data retention to increase the endurance of MLC flash based SSDs.

\subsection{Related Work}

There are several transistor-level studies on charge trapping and detrapping of flash that have shown the benefit of benefit of recovery periods in improving NAND flash endurance and data retention $[55,103,104]$. The effect of temperature on interface trap generation in CMOS transistors have been theorized using concepts like Negative Bias Temperature Instability (NBTI) and Positive Bias Temperature Instability (PBTI). Similar to recovery due to detrapping in Floating Gate Transistors, traps in CMOS transistors can be removed by applying voltage bias to the transistor terminals during runtime $[3,46,85,94]$. Mohan et al. propose leveraging these properties at the architecture level to boost endurance [60]. Wu et al. propose to accelerate the recovery process using heat [71], although heat-assisted detrapping leads to data loss and therefore requires data to be backed up and reloaded into flash based storage devices after heating.

\subsection{Summary}

In this chapter, we have presented a detailed analytical model to understand data retention based NAND flash failure mechanism. We use the model to quantify the impact of recovery period and temperature on data retention. We show that recovery periods can significantly boost the data retention of NAND flash based memories and is an important parameter to be considered for optimization. We also show that high temperatures negatively impact NAND flash data retention due to 
increase in Stress Induced Leakage Current (SILC). The relationship between temperature and data retention is modeled using an Arrhenius equation with an activation energy of $0.3 E V$. 


\section{Chapter 4}

\section{Building High Endurance, Low Cost SSDs for Datacenters}

\subsection{Introduction}

As large enterprises move to the cloud, they host online services and store consumer data in large scale datacenters across the world. Moving consumer data to the cloud increases the demand for both storage capacity and performance. Storage accounts for a significant portion of the Total Cost of Ownership (TCO) and contributes upto $30 \%$ of the total power consumption in a datacenter [20]. This number is projected to become more significant given the uptrend in user data being stored on the cloud. IDC reports that user data stored in datacenters has been growing at an exponential rate [21]. Given the increasing demand, the cost of designing storage is expected to increase significantly. In order to keep pace with increasing data growth, enterprises are forced to look for new storage technologies that can achieve performance and capacity requirements at an optimal cost.

Solid State Drives (SSDs) have become increasingly relevant for datacenter storage since they achieve these objectives. The $\$ / G B$ of flash based devices has been dropping steadily, making them attractive for use in large scale storage systems. SSDs offer several advantages including lower power and higher performance when compared to hard disk drives that were the traditional storage medium in large datacenters. Since enterprise workloads are mostly random in nature [75], they benefit significantly from the superlative random I/O performance of SSDs. Large datacenters hosting online services, such as Web Search, OLTP, and Cloud services employ SSDs as main memory extension buffers, Hard Disk Drive (HDD) caching solutions in the storage hierarchy, and 
sometimes as actual HDD replacements. Flash memory comes in three flavors - (i) Single Level Cell (SLC), (ii) Multi-Level Cell (MLC), where multiple bits are stored per memory cell, and (3) Enterprise MLC (eMLC). SLC provides the highest performance, but is the most expensive and least dense of all three types. MLC provides the lowest cost and highest density, albeit at a lower performance than SLC. However, MLC is still a strong performance value proposition compared to HDDs, since HDD performance is still significantly lower than MLC flash. eMLCs are MLC based devices with additional firmware and ECC geared towards enterprise class customers but at higher cost than MLC [65]. In a datacenter scale system, a key figure-of-merit is the the ratio of the cost of the SSD to the cost of the overall server solution without SSDs. It is important to minimize this ratio in order to be able to deploy SSDs at a large scale in a cost-effective manner. Figure 4.1 presents this ratio for the three types of flash SSDs for both commodity and enterprise class servers whose configurations are shown in the table. The cost prices for the servers and SSDs are obtained from list prices available online [6]. As we can see, among the three types of flash, MLC is clearly the best in terms of the SSD:Server cost ratio for both commodity and enterprise servers.

In this chapter, we evaluate MLC based solutions, given its performance and cost benefits. However, the major impediment in adopting MLC SSDs at datacenter scale has been the endurance limitation. Enterprise storage has to support significant write traffic, when compared to client or desktop storage. This requirement directly impacts the endurance limits of flash technology. Flash memory blocks can wear out after a certain number of write (program) and erase operations, a property referred to as limited write endurance. Typical enterprise servers are designed to be costamortized for a period of 3-5 years based on the respective TCO model that an enterprise adopts. Based on this expected lifetime, replacement and service costs are projected to be $15 \%$ of the overall server infrastructure cost [98]. Hence, increasing the cost of the server due to costly SSD replacements impacts the financial bottom line of a large enterprise. If the write endurance limit is reached before the expected TCO lifetime of a server, then this results in a significant cost to the enterprise. In a cloud environment, where efficiency of operation is key to revenue margins, failures due to write endurance limitations need to be avoided. Hence there is a need for a flash device that is cost optimal and provides all the benefits of flash technology, but at the same time, does not have 


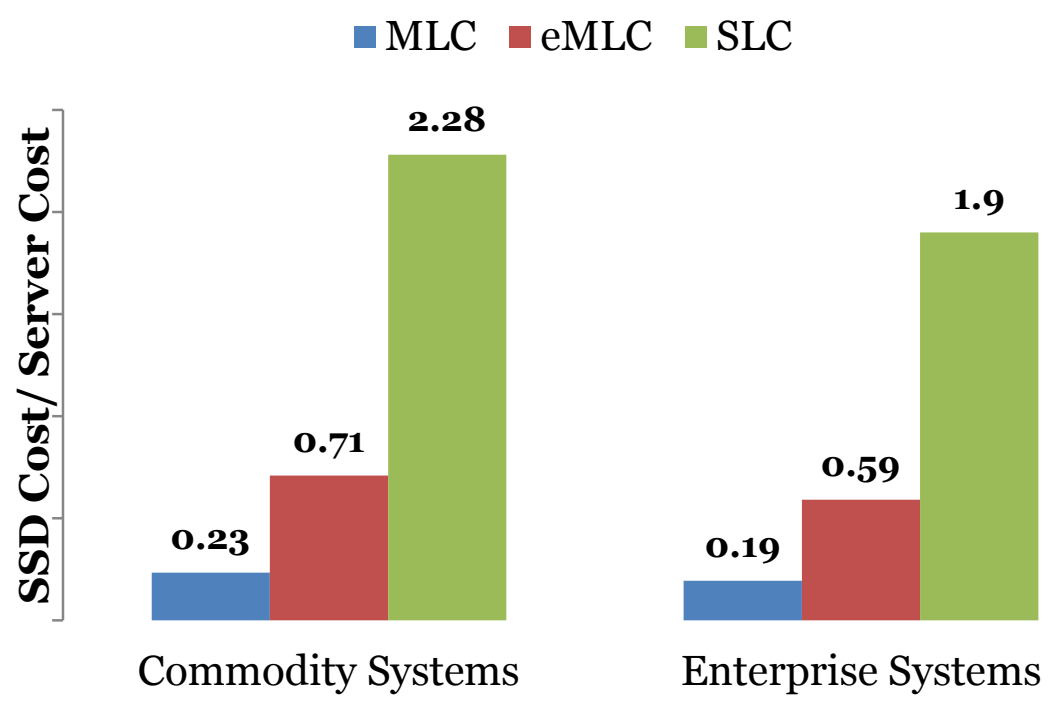

\begin{tabular}{|c|c|c|}
\hline & Commodity Systems & Enterprise Systems \\
\hline CPU & Dual (8-16 cores) & Dual (8-24 cores) \\
\hline Memory & DDR3 (24GB-32GB) & DDR3 (48G-128GB) \\
\hline Disk & $\begin{array}{c}\text { Direct Attached SATA } \\
\text { (4TB-8TB) }\end{array}$ & SAS (6TB-12TB) \\
\hline SSD & $\begin{array}{c}5 \% \text { of capacity } \\
\text { (Avg: } 300 \mathrm{~GB})\end{array}$ & $\begin{array}{c}5 \% \text { of capacity } \\
\text { (Avg: 450GB) }\end{array}$ \\
\hline $\begin{array}{c}\text { System Cost } \\
\text { (without SSD) }\end{array}$ & $\$ 2,000$ - $\$ 3,000$ & $\$ 6,000-\$ 10,000$ \\
\hline $\begin{array}{c}\text { MLC } \\
\text { Avg \$/GB : 1.94 }\end{array}$ & $\$ 582$ & $\$ 873$ \\
\hline $\begin{array}{c}\text { eMLC } \\
\text { Avg } \text { /GB : 5.91 }\end{array}$ & $\$ 1,773$ & $\$ 2,660$ \\
\hline $\begin{array}{c}\text { SLC } \\
\text { Avg \$GB : 19 }\end{array}$ & $\$ 5,700$ & $\$ 8,550$ \\
\hline \multicolumn{2}{|c|}{} \\
\hline
\end{tabular}

Figure 4.1: Cost analysis of SSDs in Datacenters. Each bar represents the ratio of the cost of a SSD to the cost of the overall server solution without SSDs. 
the disadvantage of limited write endurance. While, among the three flash technologies, MLC has the lowest SSD:Server cost ratio, it is also the one that has the least amount of endurance. The key objective of this chapter is to demonstrate how we can leverage MLC based NAND flash in datacenters while boosting its endurance. We use the reliability model developed in Chapter 3 to build high endurance SSDs by trading off data retention. To ensure that sacrificing data retention does not violate the integrity of the stored data, we propose that the flash memory cells be periodically refreshed and we evaluate the performance and reliability impact of implementing an Earliest Deadline First (EDF) based refresh policy within the SSD for a set of datacenter workloads.

The rest of the chapter is organized as follows: Section 4.2 describes the experimental setup while Section 4.3 describes how we simulate Enterprise SSDs using Hard-Disk Drives (HDDs) based workload traces. In Section 4.4, we propose a new methodology to evaluate SSD lifetime over long time periods (in the order of years), while Section 4.5 explains the benefits of the EDF based refresh policy on the reliability of MLC based SSDs. Chapter 4.6 presents the related work and Chapter 4.7 summarizes this work.

\subsection{Experimental Methodology}

In this section we describe our experimental methodology for analyzing SSD performance and reliability.

\subsubsection{SSD Configuration and Simulator Setup}

As our objective is to evaluate the reliability of MLC in datacenters, we simulate a 64GB 2-bit MLC based SSD (M-SSD) similar to [31]. M-SSD uses a wear-aware cleaning algorithm and uses free blocks within a memory chip for wear leveling (allocation pool granularity of a chip) as mentioned in [5]. M-SSD has a spare area of $10 \%$ and also supports internal plane level copy back operations to leverage plane-level parallelism available inside SSDs. We use Disksim, a widely used trace driven simulator for studying storage systems, augmented with with an SSD model developed 


\begin{tabular}{|c|c|c|c|c|}
\hline Workload & $\begin{array}{c}\text { Trace } \\
\text { Duration } \\
\text { (hours) }\end{array}$ & $\begin{array}{c}\text { Total } \\
\text { I/Os } \\
\text { (millions) }\end{array}$ & $\begin{array}{c}\text { Read \& } \\
\text { Write } \\
\text { ratio }\end{array}$ & $\begin{array}{c}\text { Request } \\
\text { Inter-Arrival } \\
\text { average(ms) }\end{array}$ \\
\hline $\begin{array}{c}\text { Display Ads } \\
\text { Platform Payload } \\
\text { Server (DAPPS) }\end{array}$ & 24 & 1.09 & $1: 1.22$ & 79.63 \\
\hline $\begin{array}{c}\text { Exchange Server } \\
\text { (Exchange) }\end{array}$ & 24 & 5.50 & $1: 2.22$ & 15.79 \\
\hline $\begin{array}{c}\text { MSN File } \\
\text { Server (MSNFS) }\end{array}$ & 6 & 2.22 & $1: 1.24$ & 9.78 \\
\hline $\begin{array}{c}\text { MSN Metadata } \\
\text { Server (MSNCFS) }\end{array}$ & 6 & 0.52 & $1: 0.64$ & 41.63 \\
\hline
\end{tabular}

Table 4.1: Properties of Enterprise Workloads Used for Evaluation.

by Microsoft [5]. We incorporate our analytical reliability model developed in Section 3.3 into Disksim.

\subsubsection{Workloads}

Our workloads consist of block-level I/O traces collected from production systems in Microsoft that use HDD-based storage and are available publicly [75]. These workloads are summarized in Table 4.1 and correspond to the Logical Unit (LUN) with the highest write traffic. Each workload trace consists of millions of I/O requests corresponding to many hours of usage, span over several terabytes of I/O address range, and spread across many Logical Units (LUNs). As program and erase operations have the highest impact on flash reliability, we choose the LUN with the highest write traffic for our analysis.

\subsection{Simulating SSDs with HDD Workload Traces}

Since SSD performance is much higher than that of an HDD, a storage system where HDDs are replaced by SSDs will be expected to be more responsive and can absorb higher amounts of I/O traffic. Therefore, analyzing SSD behavior using HDD traces can lead to inaccurate assessments. There are two alternatives to address this problem. One approach is to use closed-loop simulation, where we recreate the entire computing infrastructure on which the traces were collected to assess 
the I/O behavior when the HDDs are replaced by SSDs. Another alternative approach is to use I/O traces that were collected on SSD-based storage. Unfortunately, neither option is possible for us since we do not have access to datacenter systems on which the traces were collected and there are no publicly available SSD I/O traces.

In this chapter, we approximate the behavior of the existing HDD traces on SSDs using the methodology proposed by Delimitrou et al. [15], who considered the problem of generating SSD traces from a HDD trace for datacenter workloads. They showed that the inter-arrival time between $\mathrm{I} / \mathrm{O}$ requests is a key parameter that needs to be considered when generating an SSD trace. Since an SSD can handle much higher I/O loads, we shorten the inter-arrival times between the I/O requests, while preserving all the other properties of the workload trace, such as the spatial distribution, the ratio of reads and writes, and the distribution of request sizes. We use a scaling factor for the interarrival time such that the SSD can handle the increase in traffic without being over-utilized. To define over-utilization, we first define performance. Performance is defined as the response time of the SSD for the $80^{\text {th }}$ percentile of the I/O requests in the higher intensity workload, normalized to the response time of the $80^{\text {th }}$ percentile of the requests in the original HDD workload trace. We define over-utilization to be the region where the Normalized Response Time (NRT) at the $80^{t h}$ percentile is greater than 3, which we find is the point when the bandwidth of the SSD has been exhausted and consequently the SSD begins to show a clear degradation in performance for our scaled workloads.

Figure 4.2 plots the variation in the NRT as the intensity of the workload is gradually increased. The area above the horizontal line in the Figure indicates the over-utilization region. We observe that each workload reaches over-utilization at a different intensity. For Exchange, DAPPS and MSNFS, M-SSD reaches the over-utilization region when the intensity increases beyond 4, 10 and 7 respectively. Hence, for Exchange, DAPPS and MSNFS we choose acceleration factors of 4,10, and 7 respectively. For MSNCFS, the NRT does not change even when the intensity increases to 15. As shown in Table 4.1, the I/O intensity of MSNCFS is so low that even at $15 \mathrm{X}$ intensity, the response time at the 80th percentile does not change. Therefore, we choose an acceleration factor of 15 for MSNCFS. For the rest of this chapter, we denote these modified higher intensity workloads 


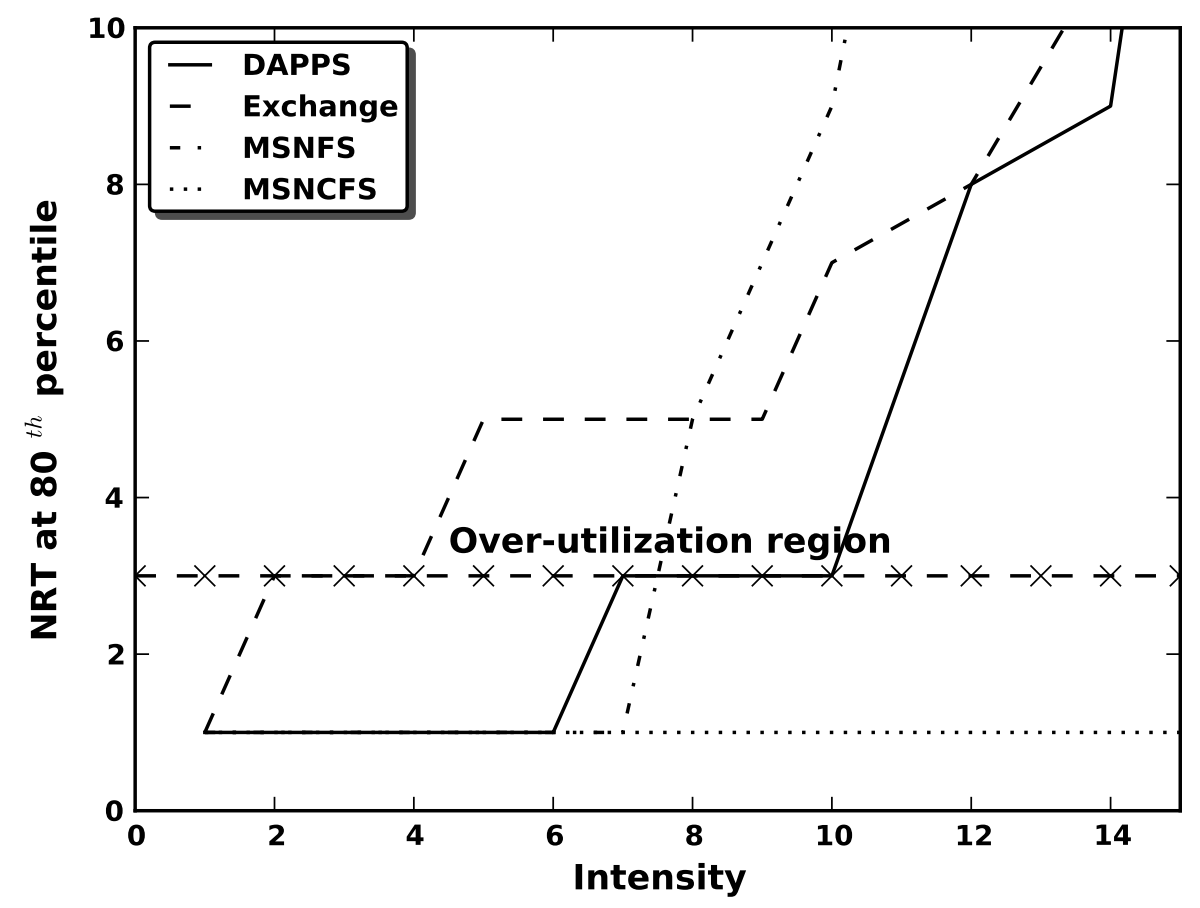

Figure 4.2: Impact of Varying Workload Intensity on SSD Response Time

as SSD-EXCH,SSD-DAPPS, SSD-MSNFS and SSD-MSNCFS while using them for analysis.

\subsection{Estimating SSD Lifetime Over Long Timescales}

Since SSD reliability issues manifest over a period of many years and TCO considerations also span 3-5 year time horizons, our analysis needs to span such time periods. In order to carry out such an analysis, we can replay the workload traces, which span several hours to a day's worth of I/O activity, repeatedly over a multi-year timescale. However, running a simulation of an I/O intense workload over such a long time period requires several weeks of simulation time and is therefore prohibitively expensive. All our high-intensity workloads except SSD-MSNCFS are very $\mathrm{I} / \mathrm{O}$ intense and therefore suffer from these excessively long simulation times. In order to tackle this problem, we use a statistical approach to reduce the simulation time for SSD-EXCH, SSDDAPPS, and SSD-MSNFS, and perform a detailed simulation of SSD-MSNCFS. We now describe our statistical approach. 
For each workload, we run a detailed simulation in Disksim using the baseline HDD workload trace as well as some high intensity versions of the trace over a multi-year timescale. We choose this timescale to be 5 years. After simulating every 15 days worth of I/O activity, a snapshot containing the lifetime of all the blocks in the SSD is obtained. Each snapshot contains the $\delta V_{t h}$, the P/E cycles, and the retention period of every block in the SSD.

We found that over this 5-year time period, the retention period of blocks in the SSD are normally distributed for MSNFS and DAPPS. Exchange had bimodal normal distribution due to a skewed spatial distribution of requests in flash chips 7 and 8 and hence those chips were wearing out faster than the other chips in the SSD. However, most importantly, irrespective of the intensity and time at which the snapshot was obtained, the type of the distribution remained the same. Because the type of the distribution is found to be independent of intensity and time at which the snapshot is obtained, determining the lifetime of blocks at a higher intensity and at a particular time requires us to estimate the mean and standard deviation of block lifetime distributions for MSNFS and DAPPS, while for Exchange, we need to estimate two means and two standard deviations corresponding to the bimodal normal distribution. (Bimodal normal distribution has a $5^{\text {th }}$ parameter, $p$, that indicates the ratio of the area of the two normal distributions. We do not estimate this parameter, because $p$ remains constant across all simulations.)

For each workload, let $S_{t, i}$ denote the snapshot at time $t$ for intensity $i$. As snapshots are stored for every 15 days of simulated I/O activity, $1<=t<=120$ (snapshots are taken every 15 days for 60 months - a total of 120 snapshots). Because simulation time increases as intensity increases, we could not run detailed simulations with the same intensity for every workload. For Exchange, snapshots corresponding to detailed simulation were obtained for $i=1$ and $i=2$, while for DAPPS, MSNFS, snapshots were obtained for $i=1$ to $i=5$. In order to get the distribution of retention period for all blocks in the SSD for SSD-MSNFS $(i=7)$ and SSD-DAPPS $(i=10)$, we need to estimate two parameters, while for SSD-EXCH $(i=4)$, we need to estimate four parameters at various time intervals. For each parameter to be estimated, we use the following approach. We use regression on a subset of data from the snapshots to obtain a curve that fits the data. Since it is possible to fit the data with more than one curve, we choose the curve that has the least root 


\begin{tabular}{|c|c|c|c|c|c|c|}
\hline \multirow{2}{*}{ Workload } & \multicolumn{3}{|c|}{ Mean } & \multicolumn{3}{c|}{ Std deviation } \\
\cline { 2 - 7 } & $\mathrm{M}$ & $\mathrm{E}$ & $\mathrm{D}$ & $\mathrm{M}$ & $\mathrm{E}$ & $\mathrm{D}$ \\
\hline DAPPS $(i=1)$ & 6.51 & 6.52 & 0.01 & 0.34 & 0.27 & 0.07 \\
DAPPS $(i=2)$ & 4.49 & 4.52 & 0.03 & 0.33 & 0.23 & 0.10 \\
\hline \multirow{2}{*}{ Exchange $(i=1)$} & 2.91 & 3.37 & 0.46 & 0.33 & 0.80 & 0.47 \\
\cline { 2 - 7 } & 3.78 & 5.37 & 1.59 & 0.19 & 2.98 & 2.79 \\
\cline { 2 - 7 } Exchange $(i=2)$ & 1.07 & 1.31 & 0.24 & 0.15 & 0.63 & 0.48 \\
\cline { 2 - 7 } & 1.73 & 3.54 & 1.81 & 0.13 & 1.93 & 1.8 \\
\hline MSNFS $(i=1)$ & 4.39 & 4.45 & 0.06 & 0.33 & 0.24 & 0.09 \\
MSNFS $(i=2)$ & 2.31 & 2.33 & 0.02 & 0.31 & 0.23 & 0.08 \\
\hline
\end{tabular}

Table 4.2: Cross validation results for extrapolated vs measured parameters. All units are in years. "M"stands for measured parameter,"E" for estimated parameter, and "D"for absolute difference between measured and estimated parameter. Exchange has 2 means and 2 standard deviations because of bimodal normal distribution.

mean square error. Each curve is of the form $z=f(t, i)$ where $z$ is the parameter to be estimated, $t$ corresponds to the snapshot time interval and $i$ is the intensity. For example, in order to estimate the mean lifetime of blocks for SSD-MSNFS $(i=7)$ at various time intervals, we use the mean lifetime of a subset of snapshots $S_{t, i}$, where $1<=i<=5$ and $1<=t<=48$ as the training data to obtain a curve which fits this data. The accuracy of the fitted curve is cross-validated by comparing the estimated parameter with the measured data which was not part of the training set. Table 4.2 shows the results of the cross-validation for two data points corresponding to $i=1$ and $i=2$ while maintaining $t$ at 72 .

From Table 4.2, we can see that the estimated and the measured parameters are very similar to each other. Specifically, the means and standard deviations of the predicted and estimated parameters are almost the same for MSNFS and DAPPS. For Exchange the means and the standard deviations of the first mode differ by less than 0.5 years. However, for the second mode of the bimodal distribution, the difference in means and standard deviations is a little higher (1.59 to 2.79 years). It should be noted that the second mode corresponds to data blocks with higher lifetime (their mean is higher than the mean of the first mode) and hence, the error introduced by the difference in estimation of the second mean would only impact blocks with higher lifetime and hence have less impact on the overall lifetime of the SSD.

We use the regression equations to estimate the age of all the blocks in the SSD for a given time 
and workload intensity. We then sample the multi-year timescale that we are interested in studying by choosing several points in time over this timescale. We then run a short duration (1 hour) detailed simulation in Disksim from each such instant to determine the impact of aging on performance for each of the workloads that use the extrapolation.

\subsection{Impact of SSD Workloads on MLC Flash Reliability}

In this section we analyze the impact of SSD enterprise workloads on the reliability of M-SSD. We begin by defining the metrics that we use for evaluation, then analyze the retention time vs. endurance characteristics for the workloads, and then evaluate the refresh policy.

\subsubsection{Metrics}

To quantify lifetime, we use the virtual retention period as the metric. The virtual retention period is defined as the minimum time duration for which any data written in the SSD can be read successfully. SSDs can have different virtual retention period requirements depending on the application for which they are used. If the SSDs cannot store data for the specified virtual retention period they are considered to be unreliable. Typical virtual retention period requirement for applications vary from 3 months to 1 year [39]. For the baseline analysis, we consider the SSD to be unusable if the actual data retention period of blocks drops below the virtual retention period. By using a range of virtual retention period thresholds (1 year, 6 months, 3 months and 1 month), we analyze the reliability of SSD over a variety of usage scenarios.

Since different blocks in the SSD can have different retention periods, we use the retention period at the $10^{\text {th }}$ percentile of all blocks as the criteria for our evaluation. We chose the $10^{\text {th }}$ percentile because, the over-provisioning capacity of M-SSD is $10 \%$ and the SSD is still functional as long as the amount of free space in the drive is more than $10 \%$. Hence, we consider the SSD to be completely unusable when the data retention period of $10 \%$ of blocks falls below the virtual retention period threshold. As mentioned in Section 4.2, we use the normalized response time of the $80^{\text {th }}$ percentile of the total number of requests as the performance metric. 


\subsubsection{Baseline Evaluation}

In Figure 4.3, we show the impact of SSD workloads on MLC SSD reliability. Our objective is to determine if our MLC SSD (M-SSD) is capable of servicing enterprise workloads over the service life. If not, we use our results to determine when the M-SSD needs to be replaced. The x-axis in the figure represents the period of simulation which extends upto 5 years (or 60 months) while the yaxis is the retention period at the $10^{\text {th }}$ percentile. The various dotted horizontal lines parallel to the $\mathrm{x}$-axis indicate different virtual retention period thresholds. The curves in the Figure 4.3 are similar to the curves in Figure 3.2b, except that the y-axis is in log-scale. While the data in Figure 3.2b is for a single flash memory cell under controlled stress-recovery conditions, the data in Figure 4.3 also takes into account the workload behavior, SSD architecture, and the FTL. Hence, the points in the curve where their slopes change are different for different workloads. From Figure 4.3, we can see that SSD-EXCH, SSD-DAPPS and SSD-MSNFS have a significant impact on the reliability of MSSD. For these workloads, the lifetime of baseline M-SSD crosses the virtual retention threshold of 1 year within 20 months of actual usage, thereby requiring multiple replacements within the TCO lifetime of the server (3-5 years). With lower virtual retention period thresholds, the usage time of the SSD increases, but this can still be improved further. Our objective is to delay the SSD replacements as long as possible, ideally beyond the TCO lifetime. The only exception to this trend is SSD-MSNCFS, whose impact on the lifetime of M-SSD is modest. Even after 5 years of use, the retention period of blocks in M-SSD is more than 3 years, indicating that the write traffic in the SSD is not sufficient enough to stress the SSD. Hence, we exclude SSD-MSNCFS from further consideration in our experiments.

It should be noted that these results take into account the stress and the recovery patterns that M-SSD experiences over its usage period. While our analysis in Section 3.4 showed that recovery periods increase the retention (and thereby the endurance) of the SSDs, these results indicate that even with the benefit of recovery periods, M-SSD cannot be used for the entire 5-year timescale for some enterprise workloads. These results were obtained after enabling state-of-the-art wear leveling optimizations like hot-cold data swap and rate-limited wearout in M-SSD [5], indicating that these existing optimizations are not sufficient to extend the usability of the SSD over the timescale. 


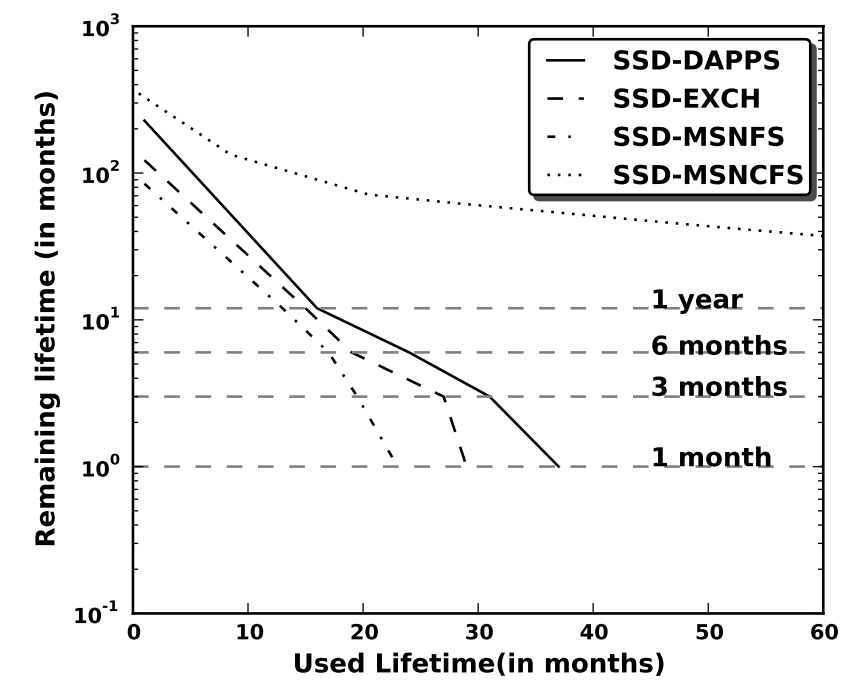

Figure 4.3: Impact of SSD Workload on M-SSD Reliability

Therefore, we require alternative approaches that can extend the lifetime of the SSDs to the maximum possible extent so as to minimize replacement and service costs and also the service downtime due to failure.

\subsection{3 reFresh SSDs - An alternative approach to increase SSD lifetime}

While trying to determine how to increase the usable lifetime of M-SSD, we observed one key point in the Figure 4.3. Figure 4.3 shows that even though the retention of some of the blocks in M-SSD is below the virtual retention period threshold, they have not yet reached their endurance limit. If we could use the remaining endurance to preserve the virtual retention of these blocks, then the usable lifetime of SSDs can be increased. In essence, what we need is a way to ensure the integrity of data stored in the underlying memory cell when the memory cell itself become unreliable.

We draw inspiration from DRAM, where data is stored in a capacitor whose inherent retention time is very short, and therefore data is preserved in the memory cells using a refresh mechanism. The refresh operation periodically restores the data in the memory cell capacitor so that no data is lost as long as the DRAM chip is connected to a power source and an intelligent scheduling of the refresh operations can minimize the performance interference of the refreshes with normal memory 
access operations. We apply this notion of a refresh to Flash memory, wherein the data in SSD blocks whose lifetime falls below the virtual retention threshold are refreshed, thereby increasing the usable lifetime of blocks. We call our SSD that use these refresh operations as reFresh-SSD.

Refreshing an SSD involves periodically checking all blocks in the SSD and moving data from less reliable blocks to more reliable blocks to match the reliability constraints of the application. Thus, a refresh operation involves a read operation of a less reliable block and a program operation to a more reliable block. Since a refresh operation is a combination of read and program operations, the only change required to support them in SSDs is to change the FTL software to initiate these operations whenever required and does not require any change to the hardware of the SSD or the flash memory chips. Refresh operations are different from wear leveling operations. Both move data across SSD blocks. But, refresh operations are triggered by the FTL when it senses that the lifetime of blocks containing data falls below some threshold (based on the application's requirement). Hence, refresh operations are triggered due to an immediate deadline, while wear leveling operations are triggered in order to achieve the long term objective of evening the wearout across blocks. The latency of a refresh operation can be significant, as it involves reading and writing all valid pages in the block. For M-SSD, this means that the latency of a refresh operation is 128 times the sum of read and write latency. Therefore, it is imperative to intelligently schedule the refresh operations so that the performance impact on normal I/O operations is minimized.

Since the need to refresh a block can be viewed as an operation that needs to be completed within a specific deadline to meet the virtual retention time requirement, we evaluate a refresh policy that is based on the Earliest Deadline First (EDF) scheduling algorithm. In this approach, the state of those blocks that contain valid data and whose lifetime is less than the virtual retention period threshold is maintained in a queue called the refresh queue. This queue is a priority queue where blocks with the lowest lifetime are at the head. Dequeue operations always begin at the head of the queue. The block with the least retention period is assigned the highest priority for the next refresh operation. Since flash does not support in-place updates, a target block needs to identified to move the contents of the block to be refreshed. To identify the target block, we use the wear leveling algorithm in the FTL to determine a free block with the highest remaining lifetime. At 
every refresh interval $r$, the FTL checks the refresh queue, picks all those blocks that need to be refreshed (refresh blocks) based on their deadline, identifies a free target block for each refresh block, copies the contents of refresh block to the target block, and finally erases the refresh block. The FTL invokes garbage collection if the number of free blocks in the SSD becomes low. In this way, even if the lifetime of a block is less than the virtual retention period, the data in the block is periodically refreshed to ensure their integrity.

Unlike traditional approaches that seek to reduce the write traffic into the SSD to increase the lifetime of SSDs, refresh operations actually increase the write traffic due to additional write operations. While this may seem counterintuitive, it should be noted that refresh operations help the FTL exploit the remaining endurance in the SSD blocks instead of ignoring them just because their lifetime is below an application determined threshold. Even though this involves additional write activity, it increases the longevity of the SSD upto a certain lifetime (which is higher than the baseline) until these additional write operations themselves decrease the lifetime of blocks in the SSD. We soon reach a point in time where the SSD spends a majority of the time refreshing its blocks, thereby heavily interfering with normal I/O operations of the workload and causing severe degradation in SSD performance. At this point, the SSD becomes practically unusable.

Figures $4.4 \mathrm{a}$ to $4.4 \mathrm{~d}$ show the benefit of using the EDF based refresh policy on the reliability of the SSD for the various workloads at different virtual retention period thresholds. The $\mathrm{x}$-axis in these figures represent the usage time while the y-axis represents the normalized response time over the usage period (lower is better). These results were obtained by running simulations upto the point where the performance of reFresh SSDs is equal to the end-of-life performance of the baseline SSDs (without refresh). Beyond this point, a significant amount of time is spent by the SSD is maintaining its state, instead of servicing the I/O requests of the workload. From these figures, we can make the following important observations:

1. Without refresh operations, SSDs with virtual retention thresholds of either 1 year or 6 months can be used for a maximum of 23 months (6-month virtual retention period for SSDDAPPS). With refresh, the usage lifetime of the SSD can be extended to upto 36 months. The increase in lifetime varies from $23 \%$ for SSD-MSNFS to $56 \%$ to SSD-DAPPS. 


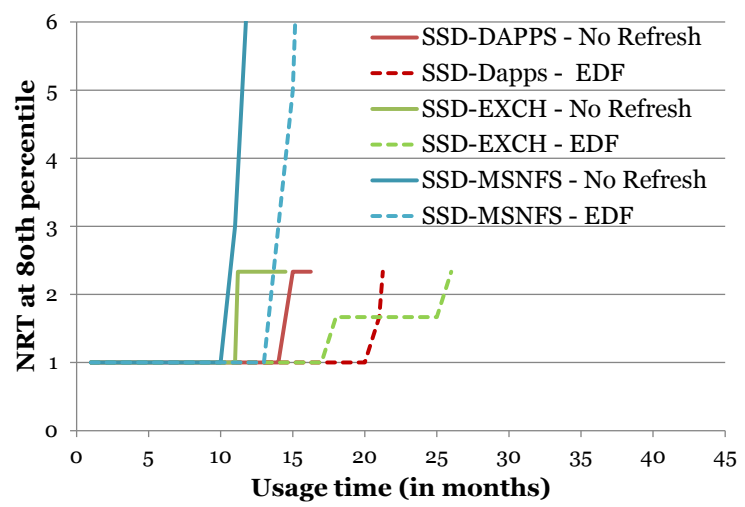

(a) Virtual retention period - 1 year

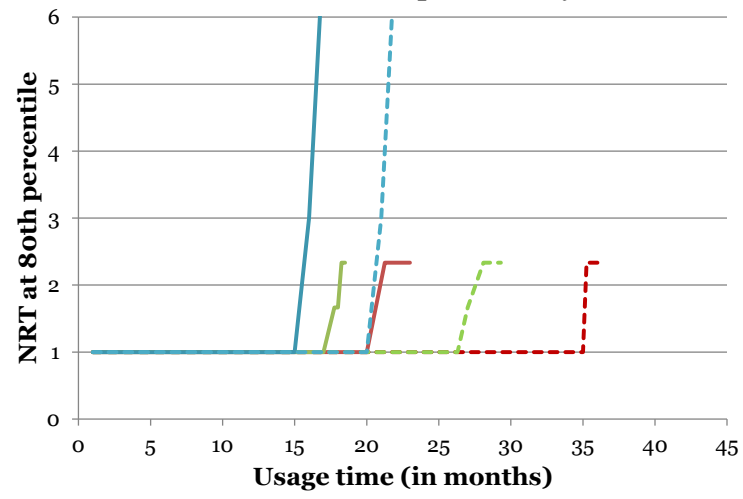

(b) Virtual retention period - 6 months

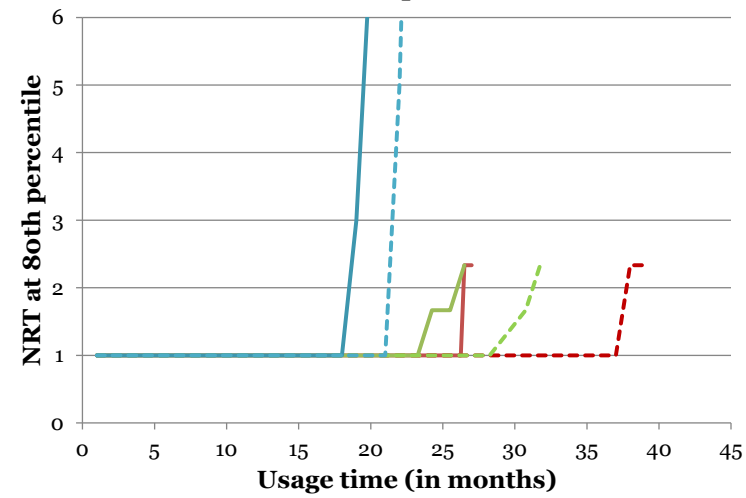

(c) Virtual retention period - 3 months

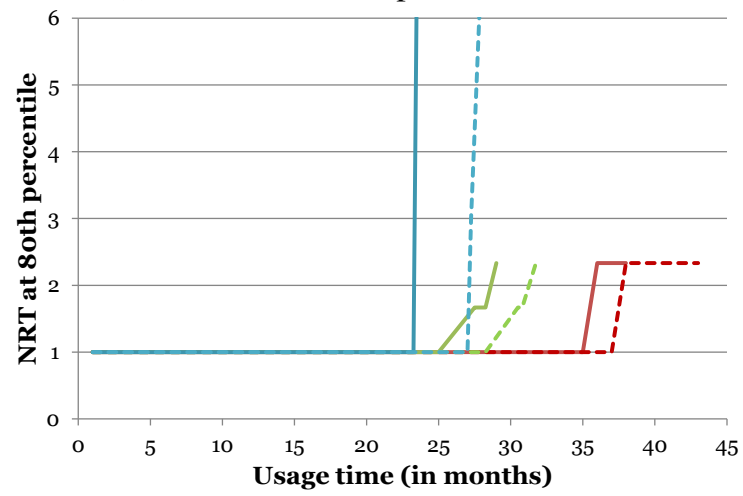

(d) Virtual retention period - 1 month

Figure 4.4: Impact of EDF Refresh Algorithm On the Lifetime of M-SSD 
2. For SSDs with lower virtual retention thresholds of 3 months and 1 month, the usage lifetime without refresh operations is a maximum of 26 months, while with refresh operations, the usage time increases to 38 months. The increase in lifetime varies from $6 \%$ for SSD-EXCH to $42 \%$ for SSD-DAPPS.

3. Reducing the virtual retention period requirement diminishes the gain in lifetime because the margin of lifetime for blocks is already becoming lower for lower virtual retention periods and blocks begin to become unusable within a relatively short time period.

4. With refresh operations, performance of the SSD varies with time. Even after the SSD crosses the baseline lifetime, there is a significant time period where refresh operations have very little impact on the performance of SSDs. This is because the lifetime of blocks is relatively high such that the number of refresh operations are performed infrequently. However, as the blocks in the SSD reach their physical reliability limit, the performance starts to degrade. We can observe that the normalized response time remains at 1 for some duration of time before starting to increase sharply and soon reaches the response time of the baseline case.

5. The benefit of refresh operations directly correlate to the intensity of the write traffic in the workloads. Workloads with lower write traffic (e.g. SSD-DAPPS) cause the SSD to fail at a later point in time and the rate at which the blocks fail is also slower compared to the more write-intense workloads. For those workloads where the rate at which blocks fail is low, fewer refresh operations are scheduled over any given interval of time. Therefore, the performance overheads imposed by these refreshes are also lower for those workloads.

In Figures 4.4a to 4.4d, we can observe that the duration between when the device is starting to fail (performance starting to degrade) and has completely been rendered unusable is in the order of a couple of months for all the workloads. This is because the wear leveler in the FTL tries to even the wearout of the flash and hence the standard deviation in the lifetime of blocks in the SSD is low. This behavior does not change with the inclusion of refresh operations because the wear leveler does not change its function. 
Overall, we find that periodically refreshing the contents of an SSD is an effective way of leveraging the tradeoff between endurance and retention time to increase the usable lifetime of the SSD. Compared to the baseline case (no refresh), refresh operations allow SSDs to operate for extended periods of time with minimal performance impact. Hence, they can help reduce the number SSD replacements over the TCO period of the server infrastructure.

\subsection{Related Work}

Many architecture and software level techniques have been proposed to improve the reliability of flash based SSDs. These include the use of hybrid HDD-SSD storage devices [89, 105], FTL optimizations [24], and the use of parity codes across a RAID of SSDs [43]. Content aware techniques that examine data patterns and reduce the number of write operations have also been proposed [13,25]. Arpaci-Dusseau et al. propose a new interface called nameless writes that allows the device to control actions like wear-leveling while obviating the need for large tables [8]. A recent paper by Smullen et al. applied the idea of refresh to another non-volatile memory - STT-RAM where they use memory cells that are designed to provide very low retention times, which provide improved performance and energy-efficiency for that memory technology when used for microprocessor caches [87]. To the best of our knowledge, this dissertation is the first to leverage the tradeoff between retention time and endurance and propose the use of refreshes to boost flash endurance.

Gaining a deep understanding of flash reliability has recently become a topic of great interest in the architecture and systems communities. There have been recent flash chip characterization studies that have shown that the endurance of flash memory chips is significantly higher than those given in datasheets $[16,23]$. There has also been a field-data analysis study from Fusion-IO on the endurance, read disturbs, and data retention of SSDs in datacenters [26]. Recent research efforts like $[68,74]$ have explored the benefits of relaxing flash retention to increase SSD performance. While this chapter also examines the tradeoffs involved in relaxing flash retention, we focus our effort on increasing flash endurance instead of flash performance. Our evaluation of flash reliability is more realistic than $[68,74]$ as our model considers the impact of recovery period on data retention 
and endurance.

\subsection{Summary}

SSDs can provide large performance and power benefits for datacenter storage. However, the limited endurance of flash memory needs to be addressed before SSDs can be practical for datacenter workloads. We have shown that the endurance of flash can be boosted if one can sacrifice retention time and that the reduction in the retention time can be offset by refreshing the data in the flash memory cells. We have shown that such refresh policies can significantly extend the usability of the SSD. Our future work in this area will be to explore other refresh policies, evaluate their implementation overheads within the flash controllers, and also study how refresh can be used in conjunction with other reliability techniques to boost SSD reliability in datacenters. 


\section{Chapter 5}

\section{Architecting Petabyte Scale SSDs}

\subsection{Introduction}

Over the past few years, enterprises across the world have rapidly been adopting NAND flash based Solid State Disks (SSDs) to meet the demand for high capacity and high performance storage systems. With the cost (\$/GB) of NAND flash dropping steadily, SSDs have become more attractive and are being used across all storage tiers in the data center [19]. NAND flash technology scaling has ensured that the $\$ / G B$ of SSDs will continue its decline for the near future [36]. In the past 10 years, flash storage device capacity has increased by a factor of 1000 (128MB in 2004 to $128 \mathrm{~GB}$ in 2014) [22]. With costs declining steadily, SSD capacities have continued to scale and current enterprise SSDs have crossed the terabyte limit. As this scaling trend continues, we can envision a future where SSD capacities approach the petabyte limit.

NAND is successfully transitioning from $2 \mathrm{D}$ to $3 \mathrm{D}$ to continue memory scaling and major NAND flash memory producers are starting mass production of 3D NAND $[29,78,96]$. With the path to memory scaling being clearly laid out, this chapter examines the path to SSD architecture scaling. Using a detailed hardware and Flash Translation Layer (FTL) architecture model, we study the scalability of conventional SSD architectures under a range of workloads. We identify and address two major bottlenecks in conventional architectures that inhibit SSD scalability.

As SSD capacities approach the petabyte limit, we observe that size of Logical to Physical (L2P) address translation table used by the FTL far exceeds the cache memory size available in 
conventional controllers. Since further increases in cache memory size in SSD controllers is impractical, we conclude that a fundamental redesign of $\mathrm{L} 2 \mathrm{P}$ tables is required to effectively use the available cache memory. As SSD capacity increases, we also observe that the processing power available in conventional SSDs is insufficient to fully utilize the thousands of flash chips available in high capacity SSDs. Many SSD controllers have only one processor to execute FTL operations and most of the processor cycles is spent on executing non-host related operations resulting in drive performance degradation with increasing capacity.

In order to address these limitations, we propose FScale, a scalable distributed processor based SSD architecture for very high capacity (petascale) SSDs. FScale uses a programmable, low power processors called Local Controller(LC) that is closer to the NAND memory package and manages the flash chips within the package. By adding a local controller to the NAND memory package, we are able to scale the compute power in a SSD without increasing the cost of the flash chips. By executing FTL operations to the local controller, we are moving NAND management closer to the NAND and freeing the main SSD controller to manage the local controllers. We also present a 2-level hierarchical L2P table scheme that works with FScale architecture and efficiently uses the cache memory available in the SSDs. Using a FScale model implementation, we compare the performance of the FScale architecture against conventional SSD architecture to show that the FScale architecture can present a path towards SSD scaling and complement memory technology scaling.

The rest of this chapter is organized as follows: We provide a background of enterprise SSD architecture in Section 5.2. In Section 5.3, we develop a detailed architecture level model of conventional SSDs and quantify the scalability limitations of this architecture in Section 5.4. Section 5.5 proposes FScale, a new architecture for petabyte scale SSDs. In Section 5.6, we compare the scalability of conventional and FScale architectures. We describe the related work in Section 5.7 and summarize the work in Section 5.8. 


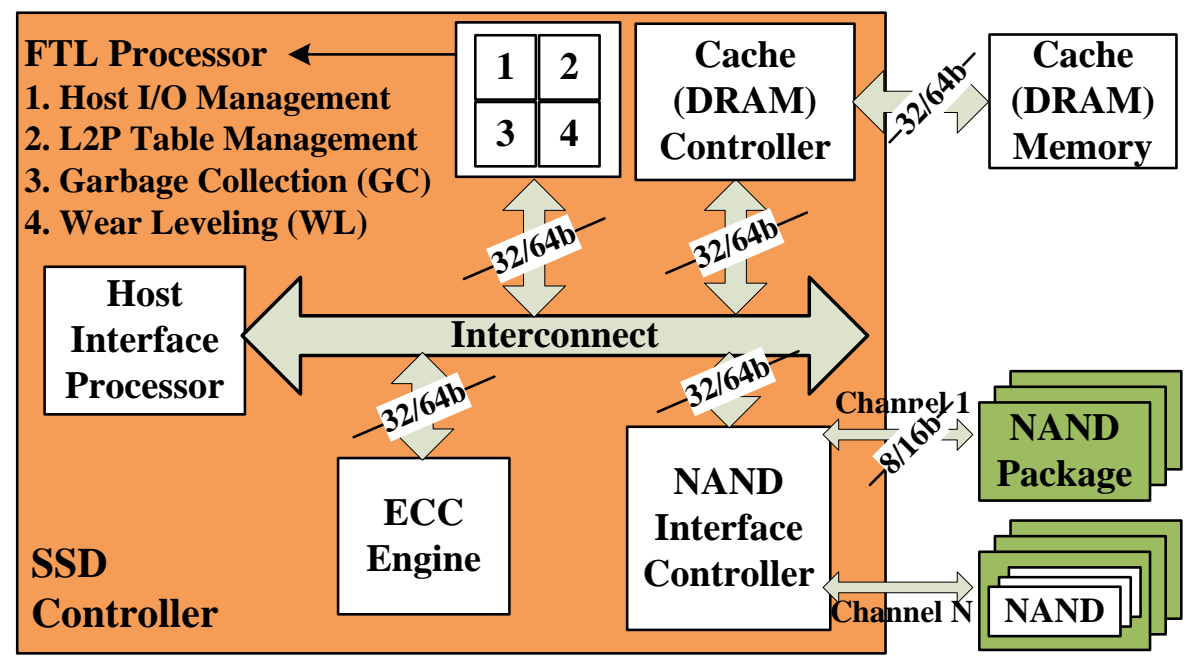

Figure 5.1: Conventional SSD Architecture. FTL Processor typically includes multiple CPU cores. Adapted based on information available from $[54,70,79]$

\subsection{Overview of Enterprise SSD Architecture}

Figure 5.1 depicts the typical architecture of an enterprise SSD modeled in this chapter. We derive this architecture from existing enterprise SSD controller products described in $[54,70,79]$. There are three major components of an enterprise SSD: an high performance SSD controller capable of meeting enterprise performance requirements, a cache (typically based on DRAM) to store host data and the Logical to Physical address (L2P) translation table and NAND flash memory chips based on the SSD capacity. Each SSD controller contains a host interface processor to process commands from the host which are based on protocols such as SATA, SAS, and PCIe. The Flash Translation Layer (FTL) processor is the brain of the SSD controller and executes major tasks like (a) managing host I/O operations, (b) managing the L2P table by paging-in or paging-out entries from/to the nonvolatile media, (c) cleaning invalid blocks in the NAND memory through Garbage Collection (GC), and (d) managing the reliability of NAND memory cells through Wear Leveling (WL). The FTL processor typically consists of one or more processor cores for performing these operations. The L2P table is cached in DRAM and is accessed by the FTL processor using the DRAM controller. Any data written to or read from the NAND flash memory is checked and corrected for errors using one or more Error Check and Correction (ECC) engines inside the SSD controller. The SSD 
controller also contains the NAND interface controller to communicate to the NAND flash memory using NAND communication protocols like ONFI or Toggle Mode. All hardware resources within the SSD controller are connected via on-chip interconnects like Advanced Microcontroller Bus Architecture (AMBA) [4], Quick Path Interconnect [73] or HyperTransport Technology [27]. Each SSD controller contains many communication lanes called channels to connect to NAND packages. The bandwidth of these channels depends on the capacitive load on them which in turn depends on the capacity of the SSD (i.e. number of NAND packages in the SSD). Each NAND package contains multiple NAND flash chips which store the data. Since NAND flash doesn't support inplace updates, SSDs are typically over-provisioned (have additional reserve pool - the physical capacity of the drive is higher than the logical capacity) so that updates can be staged to the reserve pool and minimize the performance penalty due to high NAND erase latency. Once the reserve pool becomes full, the FTL executes GC operations and compaction to free up some reserve pool capacity. During garbage collection and compaction, NAND memory experiences more writes in addition to the host writes. This is referred to as write amplification and the Write Amplification Factor (WAF) is a function of over-provisioning space. Luojie et. al provide a analytical expression to estimate WAF as a function of over provisioning [53]. SSDs typically treat the physical address space and the over-provisioned space as a common generic pool and do not differentiate between them.

\subsection{Modeling Conventional SSD Architectures}

In this section, we describe the model that we have built to study conventional SSD architectures.

\subsubsection{Modeling Methodology}

We use a SystemC based event driven simulation infrastructure developed using Intel CoFluent Studio to build a SSD model [28]. The model can be broadly categorized into two parts: hardware model and FTL model. We now explain both in detail. 


\subsubsection{Hardware Model}

The major hardware parameters that we model include the clock frequency of the FTL processor, the bandwidth of hardware resources like DRAM cache memory, ECC engine and the NAND communication channel. We account for parallelism in hardware resources by modeling parameters like number of CPU cores in the FTL processor, the number of NAND communication channels and the number of NAND flash packages and chips present in the SSD. We have incorporated a detailed NAND flash memory model which includes parameters like operation latency (for reads, writes and erasures), and the size and dimension of the memory array. As host requests arrive at the host interface processor and traverse through various hardware resources, we model the latency of the request at each hardware unit by taking the bandwidth and parallelism of that unit into account. Communication channel between hardware resources like the on-chip interconnect and the NAND channels are modeled as hardware queues with queuing delays based on the type of channel and the request.

\subsubsection{Flash Translation Layer (FTL) Model}

We have implemented a detailed FTL model that executes major tasks listed in Figure 5.2. In order to meet the performance requirements of enterprise application, the FTL model uses a page-based L2P table to map logical addresses to NAND physical addresses similar to other studies like [9, 24]. Garbage Collection (GC) algorithm in the FTL model is controlled using Write Amplification Factor (WAF) as a modeling parameter. For example, a write amplification of $\mathrm{X}$ indicates that for every host write, there are X-1 garbage collection writes and X-1 garbage collection reads. With WAF and the workload write intensity as an input to the model, the FTL model determines the number of garbage collection reads and writes to be triggered. For the FTL model, the source block (i.e. the block to be garbage collected) and the destination block (i.e. the block where the valid data should be written) are typically chosen based on a Wear Leveling (WL) algorithm. We instead assumed these blocks to be randomly distributed across the entire physical address space. The FTL model uses a scheduling algorithm to schedule host operations, garbage collection and wearleveling operations to minimize resource contention and maximize SSD performance. However, 
in cases where resource contention is unavoidable, the FTL model faithfully blocks requests from proceeding ahead until the resource is freed up. As SSD capacities increase, the size of DRAM cache required to cache the logical to physical address translation table (L2P table) becomes a major bottleneck. We have a cache-aware FTL model that includes cache miss-rate as a parameter to model the overhead associated with paging L2P entries in/out of the cache.

\subsubsection{Modeling FTL Latency}

The FTL code executes on the multi-core FTL processor and consume processing cycles for each of the operation it performs. Figure 5.2 illustrates the various phases depending on the operation type. In phase 1, the FTL sets up the data structures required for the various operation depending on the operation type (host, GC or L2P) and whether it is a read, write or erase. The scheduler and dispatcher then process the pending operations as they progress through various hardware units in the SSD. Once the operations complete, the FTL executes the cleanup phase by either notifying the host with the completed operation or by completing the GC or L2P operation. When the GC operation is complete, the engine determines whether additional blocks need to be garbage collected or the GC operations can be terminated. Similarly when the L2P operation is complete, the completion phase involves notifying the scheduler that operations that are waiting for a valid L2P entry can be scheduled. In order to model the processor cycles consumed by the firmware stages mentioned above, we have profiled the latency of each FTL operation type and phase listed in Figure 5.2 on real enterprise SSDs ranging from 100GB to 960GB in capacity [81]. While the overall firmware of these SSDs are complex and geared towards meeting the rigorous requirements of enterprise applications, their hardware and FTL architecture are similar to the one shown in Figure 5.1 and described in Section 5.3.1.2. By using FTL processor cycles, not absolute latencies, measured from profiling real SSDs, we are able to use the model to study SSD performance even with FTL processors of different frequency. By using such a modeling methodology, we also make the implicit assumption that irrespective of whether the FTL processor frequency is $400 \mathrm{MHz}$ or $5 \mathrm{GHz}$, each FTL operation type and phase requires the same amount of cycles.

Table 5.1 presents a list of major parameters that we use in modeling the SSD controller. 


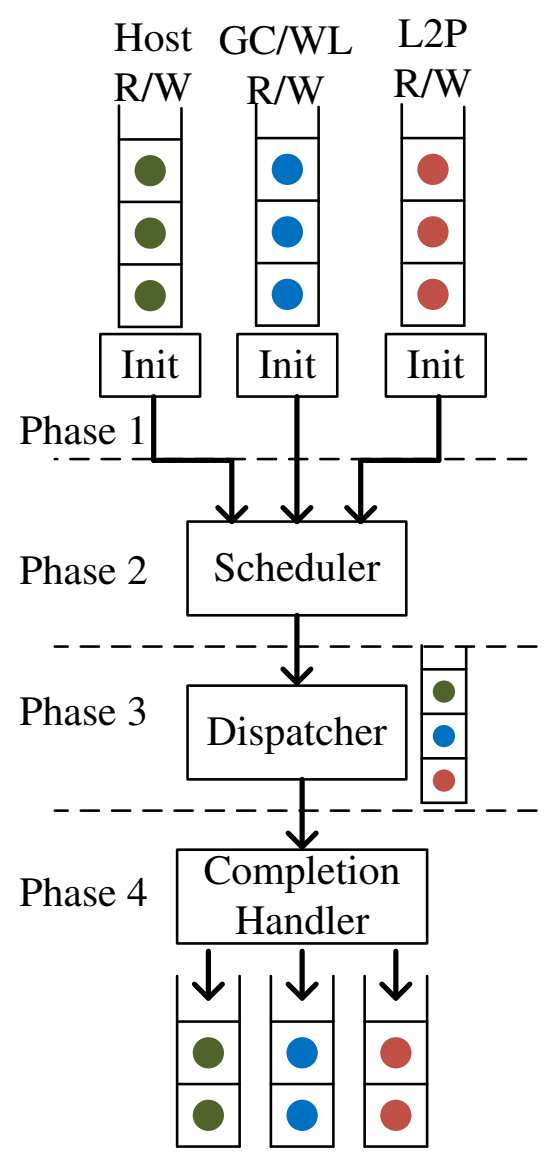

Figure 5.2: FTL Operation Types and Phases.

This figure illustrates three major operation types: host operation, GC operation and L2P table management and four phases (phase 1 to phase 4). R/W indicates reads or writes. 


\begin{tabular}{|c|c|}
\hline Category & Parameters \\
\hline SSD Controller & $\begin{array}{c}\text { FTL processor frequency, num processor } \\
\text { cores, DRAM size and bandwidth, ECC } \\
\text { bandwidth, Num channels and channel } \\
\text { bandwidth. }\end{array}$ \\
\hline FTL & $\begin{array}{c}\text { Write amplification factor, page-based } \\
\text { FTL and logical page size, cache hit rate, } \\
\text { cycles per FTL operation based on } \\
\text { operation type and phase (Figure 5.2). }\end{array}$ \\
\hline NAND & $\begin{array}{c}\text { NAND chip capacity, Num NAND } \\
\text { chips per package, NAND performance } \\
\left(t_{\text {prog }}, t_{\text {read }}, t_{\text {erase }}\right)\end{array}$ \\
\hline Workload & Read/write mix, Host I/O queue depth \\
\hline Host-Interface & $\begin{array}{c}\text { Host line rate based on protocol } \\
\text { (SATA, SAS or PCIe) })\end{array}$ \\
\hline
\end{tabular}

Table 5.1: Major parameters for the SSD architecture model.

\subsubsection{Modeling Accuracy and Relevance}

We compared the performance estimates from our model with measurements from real enterprise SSDs with capacities ranging from 100GB to 960GB [81]. Results from this analysis show that the estimates from the model were between $77 \%$ (for write-only workload) to $89 \%$ (for read-only workload) compared to estimates from the real drives. Even though parameter values for WAF, cycles per FTL operation are obtained from experiments with real drives, the FTL model and the SSD architecture are designed to be generic and does not faithfully replicate the FTL algorithms in real drives. We attribute the deviation of model estimates to this factor. While our FTL model is similar to publicly available SSD simulators like [5], we believe this is the first work that provides a detailed hardware model for the SSD by taking various hardware resources in the SSD controller into account. Since we model a generic FTL and a general SSD architecture which is not specific to any SSD controller implementation, we consider the model to be a resonable representation of conventional SSD architectures. 


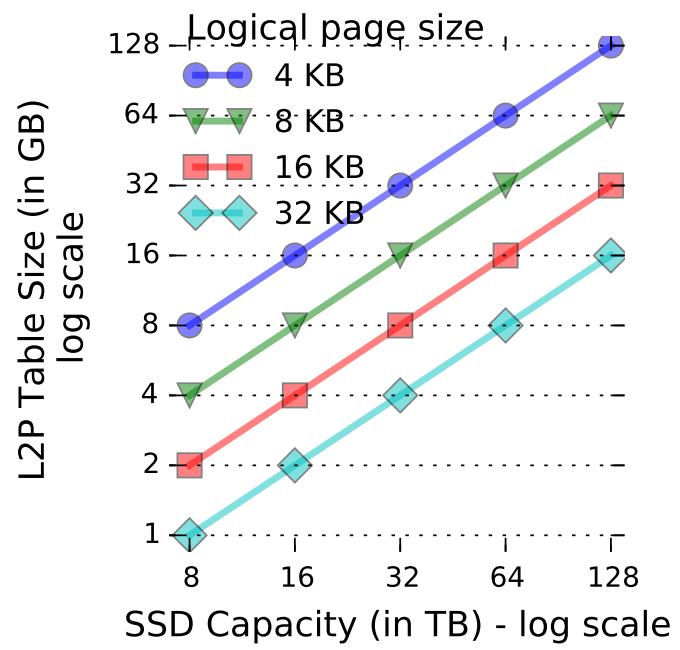

Figure 5.3: L2P Table Size as a function of SSD Capacity and logical page size.

Observations: (i) L2P table size grows with SSD capacity. (ii) DRAM cache capacity cannot scale with the table size, so cache miss rate increases with table size and SSD capacity.

\subsection{Scalability Challenges in Conventional SSD Architectures}

\subsubsection{Metrics, Workloads}

We use SSD throughput measured in I/O Operations per Second (IOPS) as the performance metric in this chapter. We also study the utilization of various SSD resources in the system to understand resource bottlenecks. All performance results in this chapter are normalized to a baseline which vary between studies and is defined at the beginning of the section describing that study. We use the term $S S D$ capacity to refer to the logical capacity of the drive to differentiate it from the $S S D$ physical capacity. In order to build versatile and robust storage systems that perform well under a wide range of conditions, customers for enterprise SSDs benchmark the drives against random I/O workloads using synthetic workload generators like Iometer [32]. Most SSD manufacturers emphasize random workload performance of their drives since the key value-add for SSDs over HDDs is their random workload performance $[30,80]$. Hence this study focuses on evaluating SSD performance as a function of 100\% random I/O workload using a synthetic workload generator similar to IOMeter. We study SSD throughput as a function of read/write ratio in the workload which varies from $0 \%$ to $100 \%$ at $25 \%$ increments and as a function of host I/O queue depth ranging 


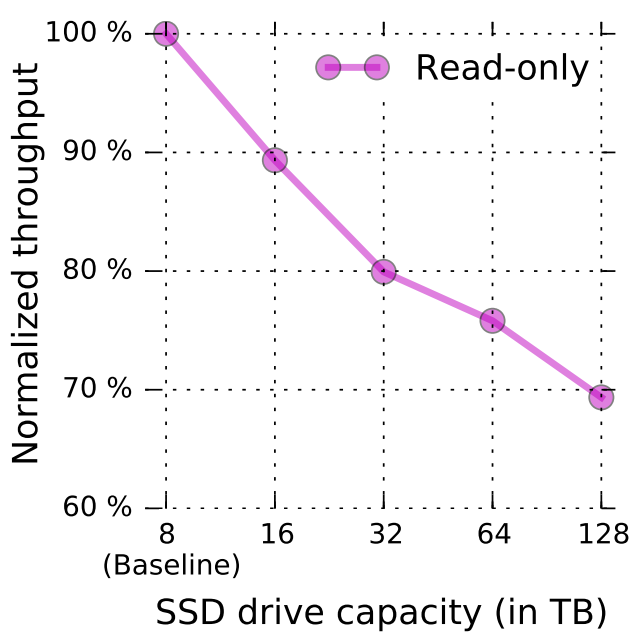

Figure 5.4: Impact of SSD capacity on random read throughput. Performance is normalized relative to 8TB. Observation: As L2P cache hit rate reduces with SSD capacity, random read workload performance degrades.

from 1 to 256. All requests from the workload generator are assumed to be aligned to a $4 \mathrm{~K}$ boundary for this work.

\subsubsection{L2P Table Size and Cache Capacity}

As SSD capacity increases, the size of L2P table required to translate logical addresses to NAND physical addresses increases significantly. In figure 5.3 we plot the size of the L2P table as a function of various logical page sizes for various SSD capacities. Given a SSD capacity (SSD logical capacity) and the logical page size, the L2P table size can be calculated based on the following equation:

$$
\text { L2P Table Size }=\frac{\text { ssd capacity }}{\text { logical page size }} \times \log _{2}(\text { ssd physical capacity })
$$

All units are in Bytes. We notice that for a fixed logical page size, the size of the L2P table doubles as capacity doubles. Similarly, for a fixed SSD capacity, the size of the L2P table halves as the logical page size is doubled. In all cases, we can observe that as capacity increases, many gigabytes of DRAM cache will be required to cache the entire L2P table. Considering that DRAM is expensive, energy intensive and consumes significant SSD board space, caching even moderate portions of L2P table is impractical. In Figure 5.4, we plot the performance impact of caching little or no portions 
of the $\mathrm{L} 2 \mathrm{P}$ table for a $4 \mathrm{~KB}$ aligned $100 \%$ random read-only workload. We have implemented a demand based caching algorithm, where table entries are paged-in from the NAND memory array upon a cache miss. A cache hit or a miss is probablistically determined using a uniform random variable that uses cache hit-rate a model parameter. We chose a read-only workload for this analysis because these workloads trigger very little garbage collection (there are no host writes) and we can clearly isolate the impact of L2P table management on SSD performance. The DRAM size is maintained at $8 \mathrm{~GB}$ while the logical page size is set to $4 \mathrm{~KB}$. Since the DRAM size is constant even

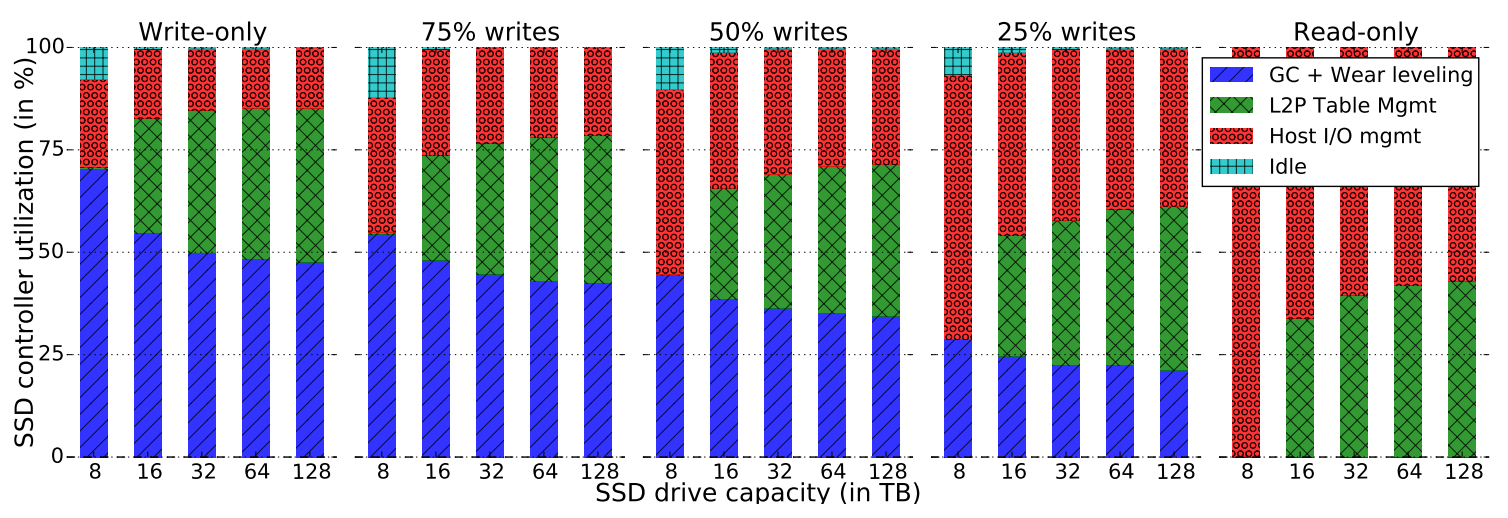

Figure 5.5: SSD Controller Utilization for major operations as a function of drive capacity and workload write intensity. Observations: (i) For all workloads, a majority of SSD controller resources are utilized for executing GC, WL and L2P page-in/page-out. (ii) As capacity scales, the fraction of resources spent on host I/O management reduces. (iii) Read-only workloads have a WAF close to 1 and hence have little GC or wear leveling operations.

as the SSD capacity varies from $8 \mathrm{~TB}$ to $128 \mathrm{~TB}$, the the DRAM cache hit rate for finding a valid L2P map (i.e. the cache hit-rate) varies from $100 \%$ to $6.25 \%$. As the capacity increases and the hit-rate drops, the throughput of the drive drops steadily. Far from being able to increase performance with SSD capacity, we notice that performance drops with capacity indicating that simple on-demand caching algorithms proposed by Gupta et al. is not optimal in the context of petascale SSDs with high cache miss rates [24]. In section 5.5.2 we propose a new table management scheme that works better with petascale SSDs. 


\subsubsection{Drawbacks in existing solutions}

Several existing studies have examined the impact of L2P table size on SSD performance and have suggested mapping schemes to reduce the size of the L2P table $[41,44,49,50]$. These studies propose increasing the logical page size to reduce L2P table by mapping logical addresses at block granularity or using a hybrid mapping approach where some sections of the table are mapped in page granularity while the rest are mapped at block granularity. These approaches increase the WAF which affect the endurance of NAND flash memory and degrades the performance of random

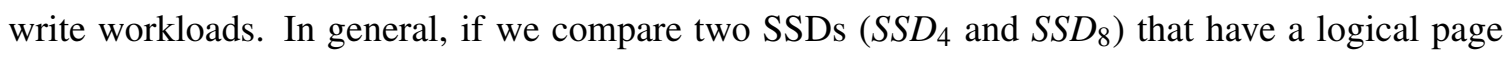
size of $4 \mathrm{~KB}$ and $8 \mathrm{~KB}$, the WAF for $S S D_{8}$ is twice the WAF for $S S D_{4}$ for the same random $4 \mathrm{~K}$ write workload. Increase in WAF impacts NAND endurance since NAND memory array would have to withstand more writes and erasures. Increasing the endurance requirement on the NAND memory array increases the time to qualify, test and optimize the memory array, thereby increasing cost and delaying time to market. In Figure 5.6, we show how WAF affects SSD performance due to high GC overhead.

Based on these results, we make the following claim.

Claim 1: As SSDs approach the petascale capacity, the page-based L2P table mapping scheme needs to be significantly modified to work with little or no DRAM cache while minimizing impact to random workload performance.

\subsubsection{SSD Controller Utilization}

In Figure 5.6, we study the impact of SSD capacity on the performance of random write workloads. We vary the write activity in the workload from $100 \%$ to $25 \%$ and plot the performance normalized to the $8 \mathrm{~TB}$ drive. For all the workloads, we assume a write amplification factor (WAF) of 5 and a logical page size of $4 \mathrm{~KB}$. Our results indicate that, similar to the read-only random workload result presented in Figure 5.4, random write workload performance reduces with capacity and is similar in magnitude. In order to identify the reason behind the performance drop, we analyzed the FTL processor utilization for various workloads in Figure 5.5 using our model. Since the FTL processor is the only central resource responsible for the initiating, scheduling, dispatching and completing 
various types of FTL tasks, the FTL processor utilization is also a reflection of SSD controller utilization (i.e. utilization of other hardware units like NAND communication channels, DRAM, ECC engine and NAND). For example, if the FTL processor executes host operations only, then the DRAM, ECC, channels and NAND are expected to only process host data. Hence we use FTL processor utilization metric as an alias for SSD controller utilization. We plot the SSD controller utilization of each FTL task as a function of workload and SSD capacity. The results from Figure 5.5 along with results from Figure 5.6 and Figure 5.4 show why SSD performance decreases with capacity.

From Figure 5.5, we can observe that for a given workload, the fraction of cycles spent by the FTL processor on executing host I/O operations reduces as SSD capacity increases. As the FTL processor spends more cycles executing non-host operations like garbage collection, wear leveling and L2P table management, the host workload performance degrades. Results from Figure 5.6 and Figure 5.4 indicate a poor tradeoff for data center operators who are forced to choose between slower but high capacity SSDs or faster but low capacity SSDs.

From Figure 5.5, we observe that for most workloads more than 50\% of FTL processor cycles is spent on non-host operations. For example, the percentage of controller cycles spent on non-host operations varies from $74 \%$ (for $8 \mathrm{~TB}$ ) to $88 \%$ (for $128 \mathrm{~TB}$ ) for the write-only workload. If host operations execute only $12 \%$ of the time in the FTL processor, we cannot expect a $128 \mathrm{~TB}$ drive to perform better than a 8TB drive. From Figure 5.5, we can observe that as SSD capacity increases, the percentage of processor cycles spent executing L2P operations increases due to the reduction in DRAM cache hit rate. For the 8TB drive capacity (irrespective of the workload), processor cycles spent on L2P table operations is close to $0 \%$ since the entire L2P table is resident on the DRAM cache. Since GC and wear leveling are a function of host write activity, as workload write intensity drops, the fraction of cycles spent on these operations drop. Hence for a given capacity, results from our model indicate that the performance of the drive increases as write intensity factor in the workload decreases. For the read-only workload, we assume WAF=1 and hence there is no garbage collection and wear leveling operations.

Based on the results presented in Figure 5.5, we make the following claim. 


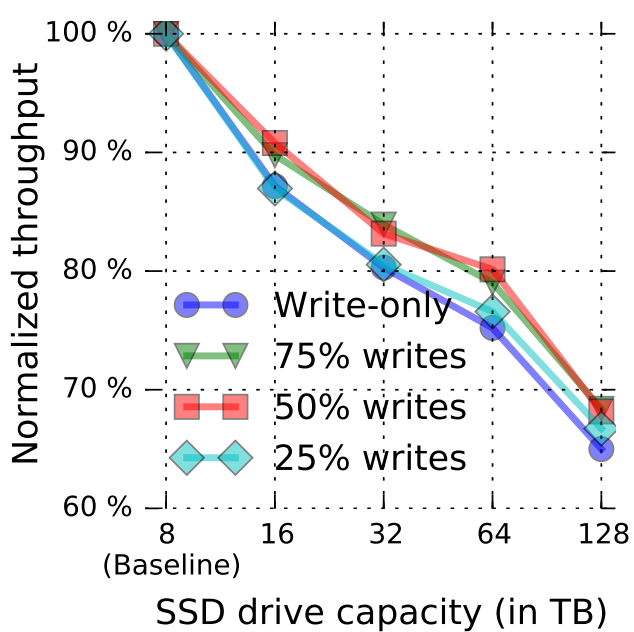

Figure 5.6: Impact of SSD capacity on random write throughput. Observations: (i) SSD random write performance scales poorly with SSD capacity. (ii) While the absolute performance varies between workloads, the rate of performance degradation is similar because results are normalized per workload with $8 \mathrm{~TB}$ as the baseline.

Claim 2: For random write workloads, most of the processing power available in conventional SSD architectures is used for GC, WL and L2P table management resulting in performance degradation of host I/O operations. Hence the processing power available in convetional SSD architectures is a major bottleneck.

\subsubsection{Challenges in Scaling FTL Processor Performance}

In the results presented so far, we assume that the FTL processor performance remains constant even as the SSD capacity scales. Such an assumption can be considered to be unrealistic because the FTL processor performance can be increased by raising the FTL processor frequency or the number of CPU cores in the FTL processor. It is perfectly reasonable to argue that by increasing the horse power of the FTL processor, the processor can spend more time executing host operations and thereby scale SSD performance with SSD capacity. Such an approach presents two major challenges: cost and time-to-market. In order to maximize profit margins, SSD manufacturers would ideally prefer to minimize the cost of non-NAND components in a SSD. By having to use a new and faster FTL processor for each capacity, the cost of the SSD increases due to higher cost of the FTL processor. Since the FTL is very closely integrated with the underlying hardware 
platform, having to rearchitect and optimize the entire architecture for each SSD capacity results in longer time-to-market. In addition to presenting significant competitive challenges due longer time-to-market, such a solution also increases SSD cost due to additional resources (like engineers, verification, assembly, qualification, etc.) required to rearchitect the system. Ideally, SSD and NAND memory manufacturers would prefer to build SSDs on top of a scalable SSD architecture where SSD performance scales up as more NAND packages are added (i.e. SSD capacity scales) without having to make significant changes to the FTL software. In this chapter, we present an SSD architecture that can scale SSD performance with SSD capacity.

\subsubsection{Host Interface Processor, ECC Engine and NAND}

The performance of the host interface processor is based on the communication protocol of the SSD (SSDs typically communicate via SAS, SATA or PCIe) and doesn't create a bottleneck. If and when the drive performance saturates the host interface, SSD manufacturers modify the host interface processor to a protocol with higher performance. Similarly, the bandwidth of the ECC engine is based to the total bandwidth of the NAND communication protocol. Since any data read from or written to the NAND needs to pass through the ECC engine, as long as the ECC bandwidth can match the total NAND channel bandwidth, the performance of the ECC engine does not limit SSD performance. NAND flash chips form the other major portion of the SSD. The state-of-art NAND flash chips are around 384Gb [29] and are expected to reach 1Tb (or 128GB) by the end of this decade [36]. At 128GB per chip, a 128TB drive is expected to contain about 1000 NAND flash chips. With so many NAND chips in the drive, we can infer that the performance of the drive is determined by the utilization of chips by the SSD controller and not on the raw performance of the chips themselves. Hence we conclude broadly conclude that the raw performance of NAND flash chips does not limit SSD performance. 


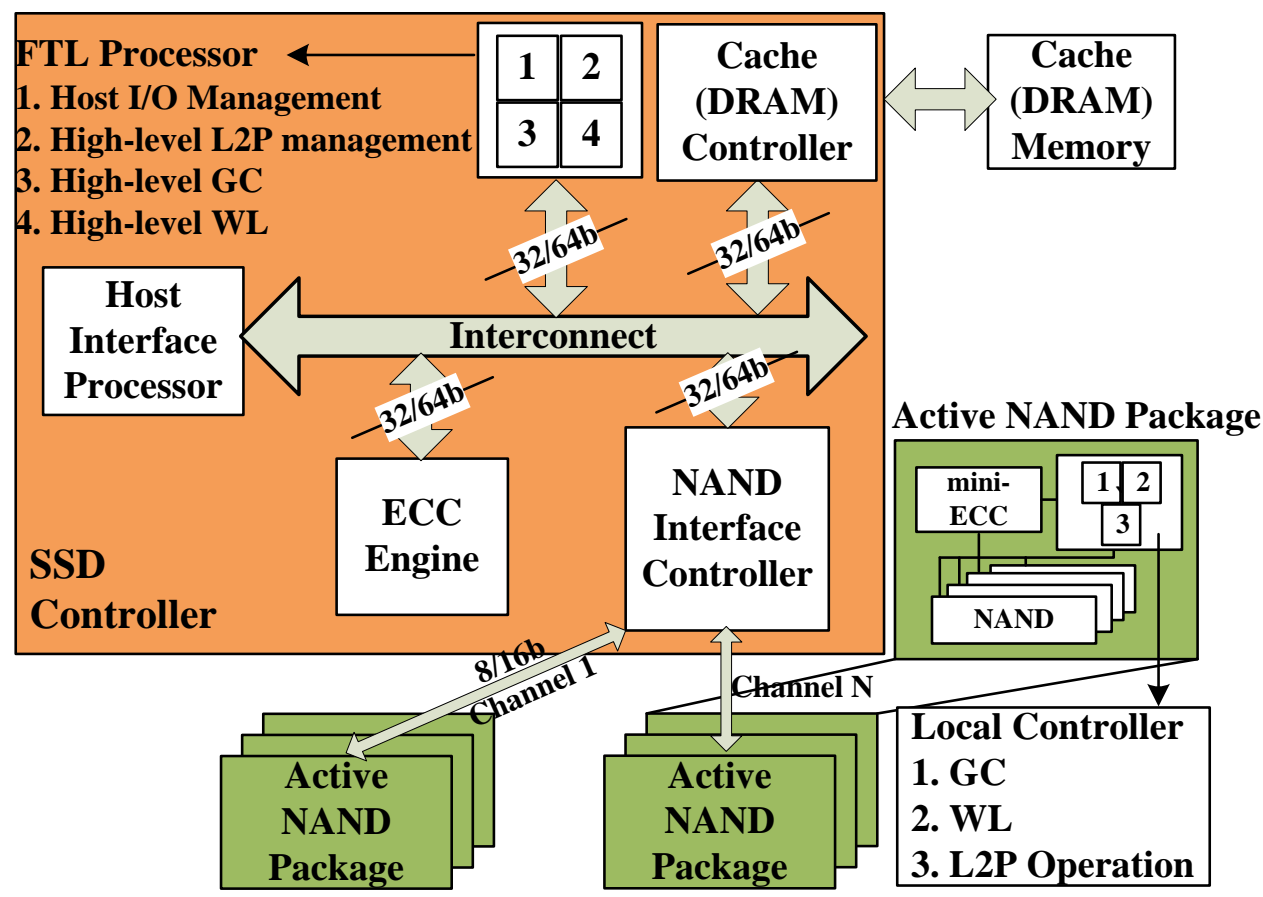

Figure 5.7: FScale Hardware Architecture
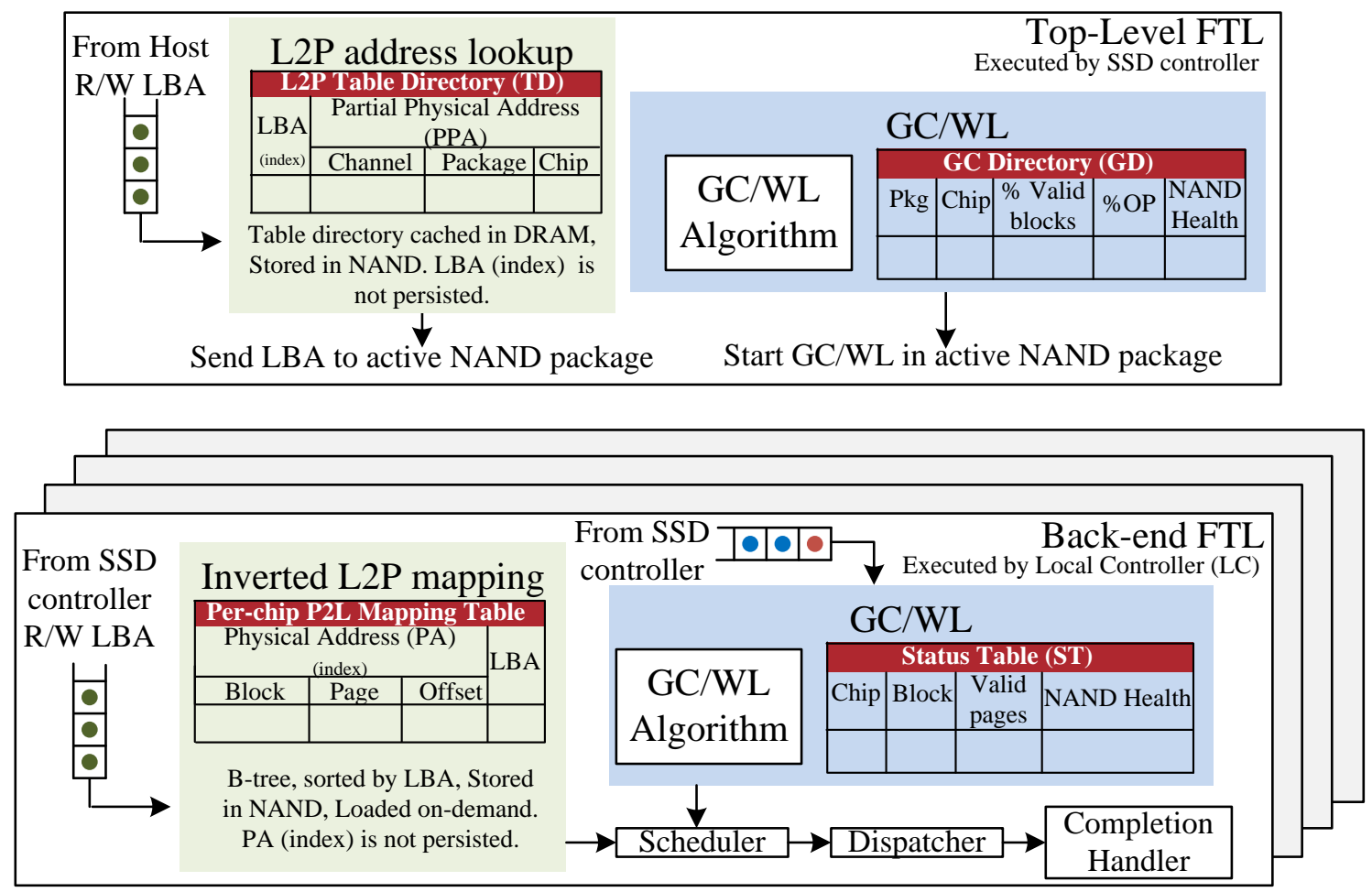

Figure 5.8: FScale FTL Architecture 


\subsection{FScale: A Scalable SSD Architecture}

We propose to address the scalability challenges in conventional SSD architectures using FScale, a distributed processor based SSD architecture targeted towards petabyte scale SSDs. Our design is based on the observation that the conventional architectures use a centralized approach to SSD management and while such an approach was optimal until now, as SSD capacity scales, the bottlenecks arising out of the centralized management prevent the system from scaling in the future.

Figure 5.7 and Figure 5.8 presents the FScale architecture. We now describe the architecture in detail and explain how it resolves the two major bottleneck claims presented in Section 5.4.

\subsubsection{FScale Hardware Architecture}

Figure 5.7 presents the FScale hardware architecture. In the FScale architecture, the NAND packages are replaced by Active NAND packages which include a local controller, a mini-ECC engine along with the NAND flash chips. Each active NAND package is essentially a mini-SSD with enough hardware resources to manage the NAND flash chips within the package. The local controller inside the active NAND package is a programmable, general purpose, lower power processor that manages the NAND flash chips inside the package. The primary purpose of the local controller is to execute major FTL tasks like garbage collection, wear leveling and L2P table management while allowing the SSD controller to execute host I/O management tasks. Our design is essentially a SSD within a SSD architecture, with the SSD controller managing the low-level SSDs within the active NAND package and the local controller in the active NAND package is managed by the local controller. Each active NAND package also contains a mini-ECC engine to handle bit errors triggered during the read and write operation in the NAND flash. A mini-ECC engine is a lowcomplexity ECC engine with a lower error detection and correction ability than the primary ECC engine in the SSD controller. Studies have shown that the bit-error distribution due to endurance and data retention in NAND flash memories has a long tail (i.e. only a very few percentage of blocks suffer from high bit error rates) [12]. The ECC engine in the SSD controller is expected to handle the long tails while the mini-ECC engine is expected to correct median bit-errors. Without 
any ECC engine inside the active NAND package, all bit errors during NAND operations need to be corrected by the ECC engine in the SSD controller resulting in unnecessary data traffic across the NAND channels. By using a mini-ECC engine in the active NAND package, a majority of biterrors can be corrected within the active NAND package, thereby minimizing data traffic across the NAND channels. During cases where the bit errors from the NAND is higher than the correction capability of the mini-ECC engine, data can be moved to the ECC engine in the SSD controller across the channel to correct the errors. To enable detection and error correction by ECC engines of two different capabilities, we need to use a multi-phase data encoding format similar to [84] that is compatible with both the ECC engines. The NAND and the local controller communicate via the NAND communication protocol like ONFI or Toggle Mode and it is assumed that the communication protocol between the SSD controller and the active NAND package is assumed to be same as the NAND protocol.

\subsubsection{FScale Hardware Architecture Assumptions}

We have built a FScale hardware architecture model by building an active NAND package model and integrating it with our SSD model. For our analysis, we assume that our local controller is similar to [7]. We assume that the bandwidth of mini-ECC engine is equal to the channel bandwidth to minimize buffering during data transfers. Since SSD drive capacity is scaled by adding more NAND packages, replacing NAND packages with active NAND packages enables us to scale the processing power available in the SSD as capacity scales up. By adding more processing power at the package-level instead of the chip-level, we prevent NAND cost from increasing so that SSD manufacturers can use such packages only for the systems that need them.

\subsubsection{FScale FTL Architecture}

Figure 5.8 presents an outline of the FScale FTL architecture. This architecture has a two-level FTL executing in the SSD. The SSD controller executes the top-level FTL while the local controller in each active NAND package executes the second-level back-end FTL. We now explain both in detail. 


\subsubsection{Top-level FTL}

The top-level FTL runs in the main SSD controller and is primarily responsible for managing the active NAND packages and handling host commands. The L2P table is also segmented into two levels with a top-level L2P Table Directory (TD) being managed by the SSD controller. While a conventional L2P table maps a logical address to a complete physical address, the table directory maps logical address to a Partial Physical Address (PPA). In the illustration shown in Figure 5.8, each logical address maps to a chip address inside the NAND package. The rest of the address mapping and execution of host operation happens at the back-end FTL. The TD is managed by the SSD controller and cached in the DRAM. We observe that the total size of the L2P mapping table (sum of TD size and second-level table) is still same as the conventional architecture but the size of TD is significantly smaller than the size of the L2P table resulting in significant increase in cache hit rate. We quantify this reduction and its impact on SSD performance in Section 5.6.

The top-level FTL executes a garbage collection and wear-leveling algorithm at the package/chip level using the GC/WL Directory table. It keeps track of valid block information and NAND health at the package/chip level and instructs the active NAND package to execute GC operation on a specific die. For example, the SSD controller does not need to know which NAND block inside the chip has been garbage collected. This in contrast to the existing architecture where the SSD controller is responsible for executing the complete operation and has to keep track of the state of GC and WL of all dies and blocks in the system and manage the operations until they finish.

\subsubsection{Back-end FTL}

The back-end FTL assumes responsibility of the same tasks executed by the SSD controller with one key difference: it is only responsible for managing the physical address space within it's NAND package and hence requires very little compute capability. The back-end FTL executes in the local controller in the active NAND package and is responsible for scheduling and dispatching garbage collection and wear-leveling operations. The back-end FTL executes address translation of logical addresses to physical addresses. When the SSD controller sends a host request to the LC, the LC reads the second-level inverted L2P table into it's working memory and scans the table for the LBA. 
Since the size of this entire table is in tens of megabytes, we load and scan partial regions of table since the working memory size in the LC is very small (a few megabytes). We use a B-tree data structure to store the Logical Block Address (LBA) in a sorted order. By using physical address as the index to the inverted table, the size of the table is bound by the physical address space of the NAND package/chip which is many orders of magnitude smaller than the logical space. Since this size is fixed and the table is organized as B-tree data structure, the number of flash memory accesses required to find a LBA inside the table is bound deterministically. In the illustration shown in Figure 5.7, we have a per-chip mapping table which is loaded and scanned for the LBA. Once a LBA match occurs, the physical address is automatically inferred and the data is read from this physical address. During writes, the local controller determines the best block to write the new data using its own wear leveling algorithm and writes data until the block is filled. When the block is filled, a new block is chosen. The back-end FTL also keeps track of the valid pages and NAND health information for all chips and blocks in the package for GC and WL using a GC/WL status table. This is similar to the tables managed by conventional SSD architecture except that this table is specific to the NAND blocks in the system. The back-end FTL implements it own scheduler, dispatcher and completion handler to execute the host, GC/WL and L2P scan operations often prioritizing host operations over the other operations to increase drive performance.

Having described the major properties of the FScale architecture, we now quantify how this architecture handles the bottleneck claims presented in Section 5.4.

\subsection{FScale Performance Results}

\subsubsection{L2P Table Size and Cache Capacity}

In Figure 5.9, we compare the size of the L2P table used in the conventional architecture with that of the L2P table directory used by the FScale architecture. Note that both these tables are cached in SSD controller cache and any reduction in size of the table can increase cache hit rate since a larger fraction of the table can be stored in the cache. We demonstrate the cache size reduction for two logical page sizes ( $4 \mathrm{~KB}$ and $32 \mathrm{~KB}$ ). Our results indicate that the $\mathrm{L} 2 \mathrm{P}$ directory is $2.7 \mathrm{X}-4 \mathrm{X}$ 


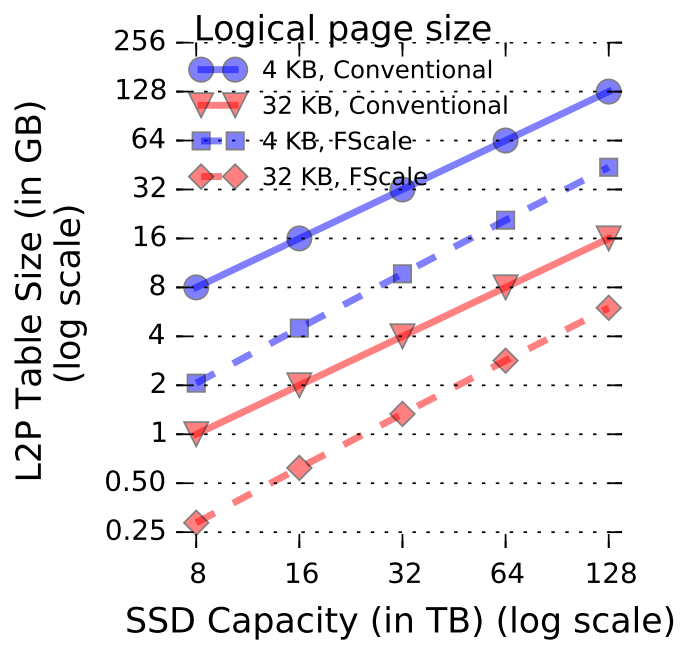

Figure 5.9: L2P Table Size Comparison between Conventional and FScale Architecture. For FScale architecture, the y-axis corresponds to the size of the L2P Table Directory (TD).

Observation: $2.7 \mathrm{X}-4 \mathrm{X}$ reduction in L2P Table Size managed by the SSD Controller

\begin{tabular}{|c|c|c|c|c|}
\hline Metric & Conv & \multicolumn{3}{|c|}{ FScale } \\
\hline Hit Rate - 8TB & $100 \%$ & \multicolumn{3}{|c|}{$100 \%$} \\
Hit Rate - 16TB & $50 \%$ & \multicolumn{3}{|c|}{$100 \%$} \\
Hit Rate - 32TB & $25 \%$ & \multicolumn{3}{|c|}{$82.5 \%$} \\
Hit Rate - 64TB & $12.5 \%$ & \multicolumn{3}{|c|}{$38.6 \%$} \\
Hit Rate - 128TB & $6.3 \%$ & \multicolumn{3}{|c|}{$18.2 \%$} \\
\hline \hline Hit latency & & min & typ & max \\
\cline { 3 - 6 } DRAM, NAND accesses & 1,0 & 1,0 & 1,3 & 1,7 \\
\hline Miss latency & & & & \\
DRAM, NAND accesses & 2,1 & 2,1 & 2,4 & 2,8 \\
\hline
\end{tabular}

Table 5.2: Hit rate, hit latency and miss latency comparison between conventional (one-level) and FScale (two-level) L2P table schemes assuming 4KB logical page size and 8GB Cache. Observation: FScale architecture improves hit rate by $3 \mathrm{X}$ at the cost of high hit and miss latency. 
smaller than the conventional L2P table. In the table directory, width of each entry is cut by $2.7 \mathrm{X}$ 4X because we truncate each entry to map only to a partial physical address (package/die offset) instead of the entire physical address (package/die/block/page/offset). By re-architecting the L2P table structure into a two-level table we can improve the cache hit rate. We have essentially applied the multi-level page table architecture present in the Linux kernel to the L2P table and let the SSD controller manage only the top-level directory [61].

Table 5.2 compares the hit rate, hit and miss latency impact of the proposed scheme against the conventional scheme. We notice significant improvements in the hit rate while the hit and miss latency of the proposed scheme is higher compared to the conventional scheme. In case of the proposed scheme, there are 3 cases for hit and miss latency depending upon whether the secondlevel table is already loaded in the local controller working memory or needs to be paged from NAND for every read access.

\subsubsection{FScale Performance Analysis}

Figure 5.10 presents the performance impact of using the FScale architecture for various random workloads. The performance of the conventional $8 \mathrm{~TB}$ drive is used as the baseline for this analysis with queue depth set to 32. First we notice that unlike conventional architecture, performance of SSDs with FScale architecture scales with capacity for write intensive workloads ( $>=50 \%$ writes). Workloads with the highest write intensity (100\% writes) have the highest performance improvement. For example, the write-only workload performance of a 128TB drive is approximately 3 times higher for the FScale architecture compared to the conventional architecture. Our results indicate similar performance improvement for other write intensive workloads indicating that the FScale architecture is successful in scaling SSD performance. We are able to scale performance because of two major reasons: (i) by offloading garbage collection and wear-leveling to the local controller, the FTL processor can spend more cycles on executing host operations and (ii) L2P cache hit rate improves with the 2-level L2P table. Even though the hit and miss latency of the L2P table scheme proposed in the FScale architecture is higher than the conventional architecture (as shown in Table 5.2), they do not impact write-intensive workloads since the write latency is one order of magnitude 


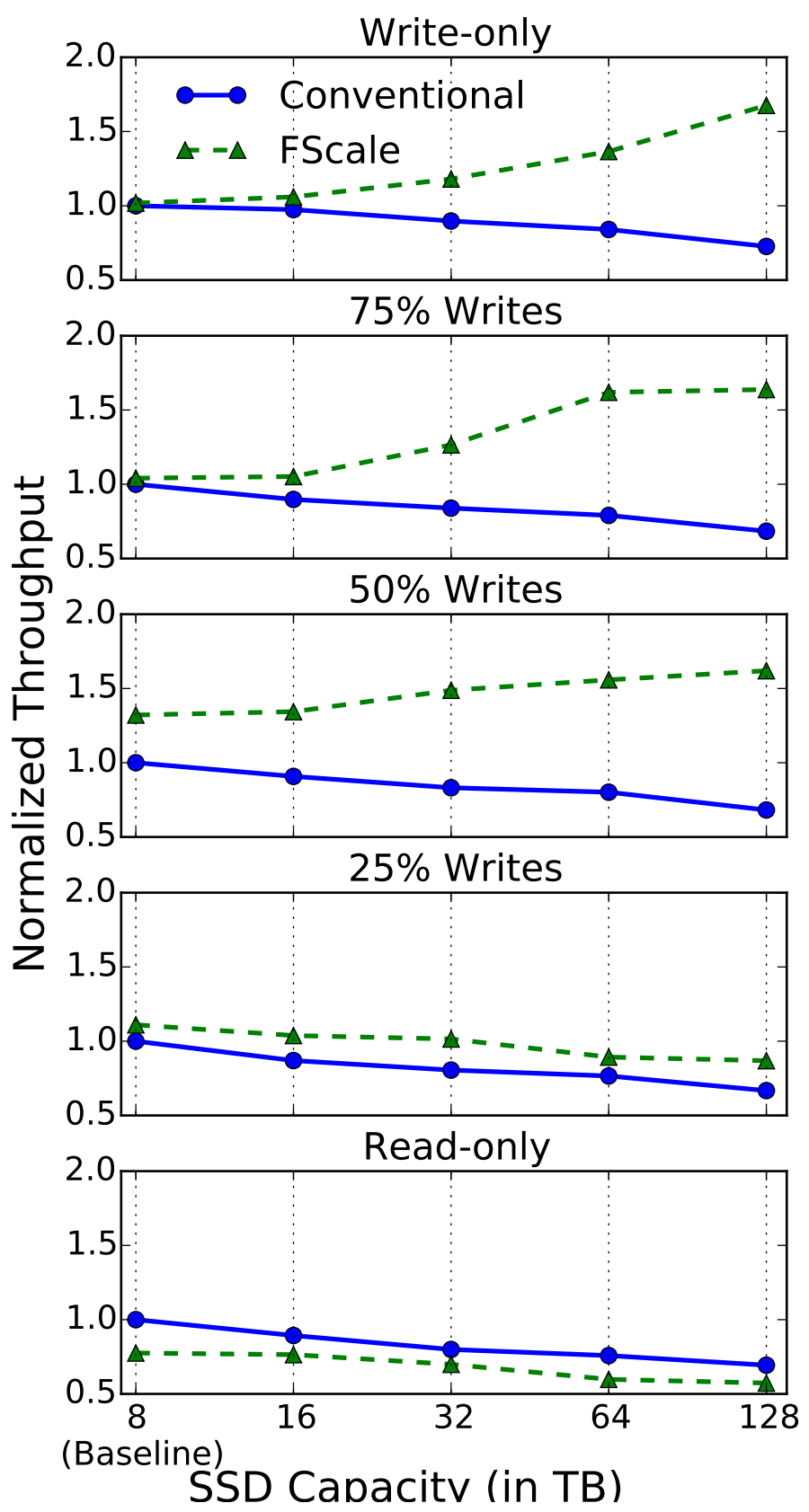

Figure 5.10: FScale Performance for various workloads

Observations:(i) For write-intensive workloads FScale SSD performance scales with capacity, (ii) For a fixed capacity, FScale performance improves by up to $3 \mathrm{X}$ over conventional architecture, (iii) Read-intensive workloads incur performance penalty due to high cache hit and miss latency even though the miss rate reduces. 
higher for writes compared to reads. Increasing the cache hit rate in the SSD controller and reducing the number of scheduling and dispatching and completion operations is much more important since they reduce the work done by the FTL processor and this translates to higher performance.

However, the higher hit and miss latencies (as shown in Table 5.2) impact the performance of read-intensive $(<50 \%$ writes) workloads. As read intensity increases, the intensity of garbage collection and wear leveling reduces which minimizes the use of the local controller. The performance of the drive will then be bound by the host I/O processing latency and L2P table management reducing the effectiveness of the FScale architecture. For the workload with $25 \%$ writes, while the performance of FScale architecture is higher than the conventional architecture, performance doesn't scale with capacity. For the read-only workload, we observe that FScale performance is lower than conventional architecture for all capacities and follows the decreasing trend indicating that hit and miss latencies have a bigger impact than the hit rate.

Figure 5.11 plots the performance of a random read-only workload for various SSD capacities. Given sufficient pending host operations, we wanted to determine if we could hide the cache hit and miss latency penalty of FScale architecture and improve workload performance. We vary the queue depth from 1 to 256 and the workload performance using the conventional architecture at a queue depth of 1 serves as the baseline. Our results indicate that the performance of the drive with conventional architecture saturate at higher queue depths while the performance of FScale SSDs increases with queue depth. As queue depth increases (i.e. number of operations pending service increases), FScale architecture can effectively hide the high cache hit and miss latency incurred by requests by efficiently pipelining the requests. Hence the performance of the drive meets or exceeds the conventional architecture.

\subsubsection{Impact of Workload Queue Depth on FScale Performance}

Our results indicate that the performance of the drive does not scale after a certain queue depth (queue depth $>64$ ) due to the compute limitations of the FTL processor in the conventional SSD architecture. With FScale processor architecture, the performance of the SSD increases with queue depth since the compute power in the SSD scales with SSD capacity. Results in Figure 5.10 for 

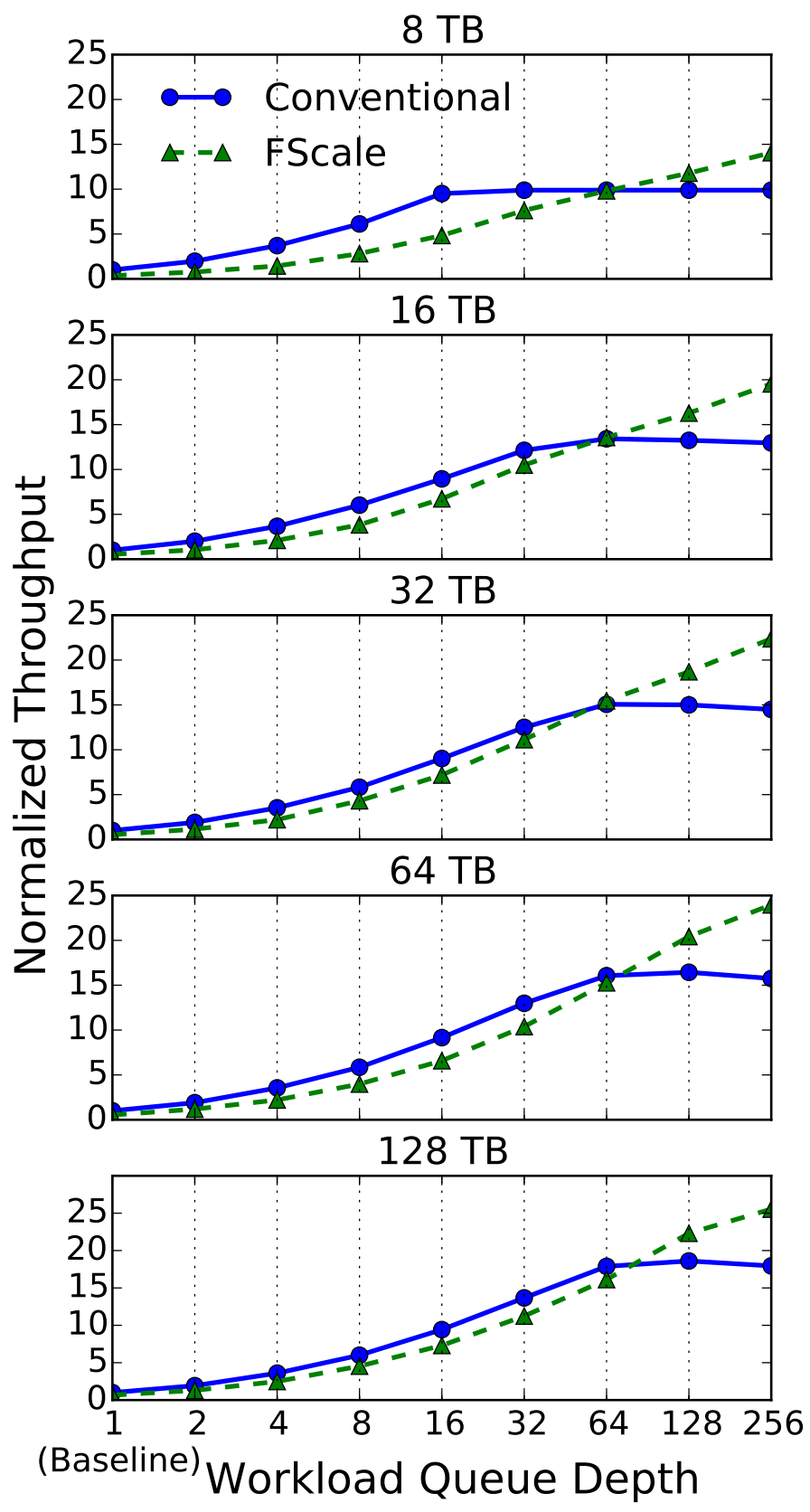

Figure 5.11: FScale Performance for Random Read-only Workloads at various queue depths Observation: For higher queue depths $(Q D>=64)$, FScale performs better than conventional architecture even for read-only workloads. 
the read only workload were at a queue depth of 32 where FScale performance is still lower than conventional SSD performance. However, we observe that at higher queue depths, the trends in Figure 5.10 do not hold and the drives tend to have a steady performance irrespective of the SSD capacity.

\subsubsection{Other Advantages of FScale Architecture}

FScale architecture helps in overcoming the cost and time-to-market challenges presented by the conventional architecture. By scaling capacity by just adding more NAND packages, SSD manufacturers can reduce the frequency at which they upgrade the SSD controller. This also reduces time-to-market. The cost added by the local controller is negligible due to its minimal complexity. While we haven't evaluated the impact of FScale architecture on the power consumption of the system in this work, features like low power processor and mini-ECC engine in the local controller are directed towards minimizing the additional power dissipation.

\subsection{Related Work}

Most of the research on SSD architecture has been towards architecting megabyte and gigabyte scale SSDs with little focus on SSD architecture scalability. Several algorithms have been proposed to optimize SSD metrics like performance and reliability and quantifying the tradeoffs between such metrics. We believe that this is the first work that focuses on the how such algorithms execute on SSD hardware and quantify the computational bottlenecks involved in executing such algorithms in the context of a typical SSD architecture.

Agrawal et al. explore the SSD architecture in detail and focus mainly on understanding the tradeoffs involved in Flash Translation Layer (FTL) algorithms and its impact on SSD performance [5]. Gupta et al. recommend a on-demand page-based address mapping policy for enterprise workloads [24] and provide insights into why high performance enterprise SSDs work better with page-based mapping instead of a block-based or a hybrid mapping policy. One of the most recent studies on SSD architecture for web-scale internet storage systems has been by Ouyang et 
al $[66,67]$. They describe a hardware/software co-designed storage system designed to exploit memory array properties of flash and is highly optimized to work for specific types of workloads. While such architectures work well for specific workloads, many customers build servers that are deployed by a wide range of customers and are expected to work well for a broad range of workloads. For such environments, the FScale architecture is a better fit since it works well for a wide range of workload categories without any workload specific optimizations. Tiwari et al. describe Active Flash, an architecture optimized towards SSDs used in supercomputers that perform in-situ scientific data analytics [95]. Their design optimizes the performance and energy efficiency of supercomputers by minimizing data movement between compute and storage nodes. For systems that use SSD as a cache in front of a hard drives, Li et al. propose a new cache-optimized SSD architecture called Nitro [52]. Nitro primarily controls the workload traffic pattern arriving into the SSD and hence is orthogonal to optimizations in FScale architecture.

\subsection{Summary}

In this chapter, we present a case for a new SSD architecture to ensure SSD performance scales with capacity. Using a detailed architectural model, we quantify the bottlenecks in existing controller architecture. We then propose FScale, a new scalable SSD architecture using a distributed processor based solution. We evaluate the performance of FScale architecture and evaluate its scalability for various workloads. 


\section{Chapter 6}

\section{Conclusions and Future Work}

The invention of NAND flash memory in mid 1980s has spawned a new category of storage systems that is versatile with high performance, low power and robust compared to Hard Disk Drives (HDDs). As flash memory scaled and cost per bit reduced, the range of applications using flash has expanded significantly. With this expanded range and diverse application requirements, systems architects are required to have a thorough understanding of the trade-offs involved in designing application optimal storage systems. By building a set of analytical and architecture level models, this dissertation presents original research contributions that is directed towards building energy efficient, reliable and scalable NAND flash based storage systems.

In Chapter 2, this dissertation presents a detailed analytical model for flash memory consumption. FlashPower has been validated with data from real world flash chips and can be used to estimate the energy dissipated by NAND flash using various operations. This model has been highly parameterized to study the impact of major parameters affecting NAND energy dissipation and enable chip architects to design power optimal NAND flash chips. While this work primarily focuses on 2D flash memory, the industry has started production of 3D flash based memory systems $[29,78,96]$. Hence, FlashPower model can be extended to study the energy dissipation of 3D based NAND flash memory chips. In this work, we focused our attention on floating gate transistors since a majority of 2D flash memory was built on top of floating gate transistors. As flash memory scales to $3 \mathrm{D}$, it has been noted that charge trap based transistors offer more advantages than floating gate based transistors. By replacing the floating gate transistor model described in Chapter 2.3.5.1 
with a charge trap based transistor model, FlashPower can still be leveraged to study the energy dissipation of 3D NAND flash chips. FlashPower provides a modularized and parameterized model that can be easily extended to study many NAND flash memory variants.

In Chapter 3, this dissertation presents a detailed analytical model to study NAND flash data retention. Using FENCE, we show the effect of temperature and recovery periods, two important system level parameters that affect NAND flash reliability, on data retention. We also show how FENCE can be used to trade-off data retention to improve NAND flash endurance. Increasing NAND flash endurance is critical for Solid State Disks (SSDs) used in data centers because of the high write activity present in enterprise workloads. In Chapter 4, we use the reliability model to improve the endurance of MLC based flash SSDs used in data centers. Since data stored in enterprise storage systems is backed up periodically, we can trade-off data retention to increase flash endurance and enable low cost and high endurance storage systems for data centers. In this chapter, we also propose a methodology to use existing hard-drive based workload traces and convert them into equivalent SSD workload traces for system evaluation. This research can be extended in several ways. With the advent of 3D NAND and the differences in failure mechanisms, it is important to identify the predominant failure modes in 3D memory and modify FENCE to understand the trade-offs involved in new failure modes. While we expect data retention and endurance to remain primary concerns, additional failure modes like read or program disturbs can impact memory reliability. It is also important to understand the impact of new failure modes on system level reliability and re-architect SSD level firmware and hardware architectures to design highly reliable storage systems.

In Chapter 5, this dissertation focuses on SSD architecture and the impediments faced by conventional architectures in increasing performance of high capacity SSDs. Using data from drivelevel measurements and a detailed architecture-level model, we show that conventional architectures scale poorly due to limited computational power in conventional architecture. As the number of NAND flash chips approach several hundreds (perhaps even thousands), we show that a centralized controller architecture prevents maximizing NAND utilization, resulting in reduced system performance. We propose FScale, a new distributed processor based SSD architecture to overcome 
this limitation and maximize NAND utilization. By integrating a small, energy efficient and low cost controller to a NAND package and managing NAND local to the package, we can scale the system efficiently and increase performance with SSD capacity. An alternative approach to increase SSD scalability is by increasing computational power in current architectures by adding more cores to the central processor. Implementing this approach requires detailed analysis to determine the core count required to manage NAND chips for an optimal design. SSD architects have to trade-off the design complexity of using a many-core processor in petascale SSDs with several distributed processors based on factors like time-to-market, power dissipation and firmware and hardware architecture complexity. FScale architecture can be extended to include one low powered, low complexity micro-controller per NAND chip instead of a controller per NAND package (a group of NAND chips). Such a system can provide better scalability because the computational power can be extended as a function of number of chips and prevent us from over designing the central processor. However, this approach increases the cost of flash chips since each chip now includes a micro-controller and can impact other parameters like chip yield and increase in design complexity. Architecting scalable SSDs is still an open problem and there can be several approaches to address this issue. In this dissertation, I present one approach to address this problem and quantify the advantages and disadvantages of the proposed architecture.

\subsection{Emerging Non Volatile Memories and Evolution of Memory Hier- $\operatorname{archy}$}

The advent of new non volatile memory technologies like Phase Change Memory (PCM) and SpinTransfer Torque (STT) RAM is expected to add a new dimension in designing memory and storage systems. NAND flash memory is not left expected to be left behind and continues to scale into 3D. As these new memory technologies mature, the 4 level conventional memory hierarchy (consisting of registers, caches, main memory and storage) will continue to evolve and expand into many more levels. For example, the performance, power and cost characteristics of PCM makes it as an good candidate between the main memory and storage memory $[1,2]$, while the characteristics 
of Spin-Transfer Torque memory makes it a good candidate to be used as a DRAM replacement or complement [88]. Such an evolution will require systems architects to work very closely with device physicists and memory designers to design optimal and efficient memory and storage systems. In this dissertation, I have applied insights learnt from the device and circuit level to optimize NAND flash memory energy dissipation and reliability while addressing SSD architecture challenges that arise with the scaling of NAND flash memory. 


\section{Bibliography}

[1] 3D Xpoint ${ }^{\mathrm{TM}}$. http://www.intel.com/content/www/us/en/ architecture-and-technology/3d-xpoint-unveiled-video.html.

[2] 3D Xpoint ${ }^{\mathrm{TM}}$. http://www.micron.com/about/innovations/3d-xpoint-technology.

[3] J. Abella, X. Vera, and A. Gonzalez. Penelope: The NBTI-Aware Processor. In Proceedings of the 40th IEEE/ACM International Symposium on Microarchitecture, 2007.

[4] Advanced Microcontroller Bus Architecture (AMBA). http://infocenter.arm.com/ help/index.jsp?topic=/com.arm.doc.ddi0438d/BABDEDDA.html.

[5] N. Agrawal and et al. Design Tradeoffs for SSD Performance. In Proceedings the USENIX Technical Conference (USENIX), 2008.

[6] Amazon.com. http://www.amazon.com.

[7] ARM Cortex M Series. http://www.arm.com/products/processors/cortex-m/ index.php.

[8] Andrea C. Arpaci-Dusseau, Remzi H. Arpaci-Dusseau, and Vijayan Prabhakaran. Removing the costs of indirection in flash-based ssds with nameless writes. In Proceedings of the 2nd USENIX conference on Hot topics in storage and file systems, HotStorage'10, pages 1-1, Berkeley, CA, USA, 2010. USENIX Association.

[9] Andrew Birrell, Michael Isard, Chuck Thacker, and Ted Wobber. A design for highperformance flash disks. SIGOPS Oper. Syst. Rev., 41(2):88-93, April 2007. 
[10] S. Boboila and P. Desnoyers. Write Endurance in Flash Drives: Measurements and Analysis. In Proceedings of the USENIX Conference on File and Storage Technologies (FAST), 2010.

[11] J.E. Brewer and M. Gill, editors. Nonvolatile Memory Technologies with Emphasis on Flash. IEEE Press, 2008.

[12] Yu Cai, Onur Mutlu, Erich F Haratsch, and Ken Mai. Program interference in mlc nand flash memory: Characterization, modeling, and mitigation. In Computer Design (ICCD), 2013 IEEE 31st International Conference on, pages 123-130. IEEE, 2013.

[13] Feng Chen, Tian Luo, and Xiaodong Zhang. Caftl: a content-aware flash translation layer enhancing the lifespan of flash memory based solid state drives. In Proceedings of the 9th USENIX conference on File and stroage technologies, FAST'11, pages 6-6, Berkeley, CA, USA, 2011. USENIX Association.

[14] Jim Cooke. Introduction to Flash Memory (T1A), 2008. http://download.micron.com/ pdf/presentations/events/FMS08_Intro_to_Elash_Memory_Jim_Cooke.pdf.

[15] C. Delimitrou, S. Sankar, K. Vaid, and C. Kozyrakis. Storage i/o generation and replay for datacenter applications. In Performance Analysis of Systems and Software (ISPASS), 2011 IEEE International Symposium on, pages 123 -124, april 2011.

[16] P. Desnoyers. Empirical Evaluation of NAND Flash Memory Performance. In Proceedings of the Workshop on Hot Topics in Storage and File Systems (HotStorage), October 2009.

[17] D. J. DiMaria and E. Cartier. Mechanism for stress induced leakage currents in thin silicon dioxide films. Journal of Applied Physics, 78(6):3883 -3894, sep 1995.

[18] Xiangyu Dong, Norman P. Jouppi, and Yuan Xie. PCRAMsim: system-level performance, energy, and area modeling for Phase-Change RAM. In Proceedings of the 2009 International Conference on Computer-Aided Design, pages 269-275, 2009.

[19] Flash-Transformed Data Center. http://www.sandisk.com/assets/docs/ the-flash-transformed-data-center-whitepaper.pdf. 
[20] Reducing Data Center Power Consumption Through Efficient Storage, 2009. http://www.gtsi.com/eblast/corporate/cn/06_2010/PDFs/NetApp\%20Reducing\% 20Datacenter\%20Power\%20Consumption.pdf.

[21] The Diverse and Exploding Digital Universe: An Updated Forecast of Worldwide Information Growth Through 2011, 2008. http://www.emc.com/collateral/ analyst-reports/diverse-exploding-digital-universe.pdf.

[22] Growth in SD Card Capacity . http://goo.gl/QcXK1R.

[23] L. Grupp and et al. Characterizing flash memory: Anomalies, observations, and applications. In Microarchitecture, 2009. MICRO-42. 2009 42nd IEEE/ACM International Symposium on, 2009.

[24] A. Gupta, Y. Kim, and B. Urgaonkar. DFTL: a flash translation layer employing demandbased selective caching of page-level address mappings. In ASPLOS '09: Proceeding of the 14th international conference on Architectural support for programming languages and operating systems, pages 229-240, 2009.

[25] Aayush Gupta, Raghav Pisolkar, Bhuvan Urgaonkar, and Anand Sivasubramaniam. Leveraging value locality in optimizing nand flash-based ssds. In Proceedings of the 9th USENIX conference on File and stroage technologies, FAST'11, pages 7-7, Berkeley, CA, USA, 2011. USENIX Association.

[26] Hairong Sun, Pete Grayson, and Bob Wood. Quantifying Reliability of Solid-State Storage from Multiple Aspects. In 7th IEEE International Workshop on Storage Network Architecture and Parallel I/O, 2011. http://storageconference.org/2011/Papers/SNAPI/1. Sun.pdf.

[27] HyperTransport. http://www.amd.com/en-us/innovations/ software-technologies/hypertransport. 
[28] Intel CoFluent Studio. http://www.intel.com/content/www/us/en/cofluent/ intel-cofluent-studio.html.

[29] Intel-Micron 3D NAND. http://newsroom.intel.com/community/intel_newsroom/ blog/2015/03/26/micron-and-intel-unveil-new-3d-nand-flash-memory.

[30] Inte SSD Product Comaprison. http://www.intel.com/content/www/us/en/ solid-state-drives/solid-state-drives-ssd.html.

[31] Intel X25-M and X18-M Mainstream SATA Solid-State Drives. http://ark.intel.com/ products/series/78570/Intel-SSD-X25-M-Series.

[32] Iometer Project. http://www. iometer.org.

[33] K. Ishida and et al. A 1.8V 30nJ adaptive program-voltage (20V) generator for 3D-integrated NAND flash SSD. In Solid-State Circuits Conference - Digest of Technical Papers, pages 238-239,239a, 2009.

[34] Process Integration and Device Structures, ITRS 2009 Edition. http://www.itrs.net/ links/2009ITRS/2009Chapters_2009Tables/2009Tables_FOCUS_C_ITRS.xIs.

[35] Process Integration and Device Structures, ITRS 2011 Edition. http://www.itrs.net/ Links/2011ITRS/2011Tables/PIDS_2011Tables.xlsx.

[36] Process Integration and Device Structures, ITRS 2013 Edition. http://www.itrs.

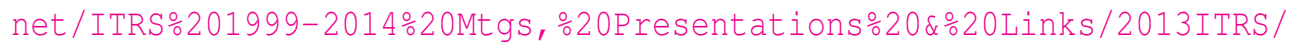
2013TableSummaries/20130RTC_SummaryTable.pdf.

[37] J. de Blauwe, J. van Heudt, D. Wellekens, G. Groeseneken, and H.E. Maes. SILC-related effects in flash $E^{2}$ PROM's-Part I: A quantitative model for steady-state SILC. Electron Devices, IEEE Transactions on, 45(8):1745 -1750, August 1998.

[38] J. de Blauwe, J. van Heudt, D. Wellekens, G. Groeseneken, and H.E. Maes. SILC-related effects in flash $E^{2}$ PROM's-Part II: Prediction of steady-state SILC-related disturb characteristics. Electron Devices, IEEE Transactions on, 45(8):1751 -1760, August 1998. 
[39] JEDEC - Solid-State Drive (SSD) Requirements and Endurance Test Method. http://www . jedec.org/sites/default/files/docs/JESD218.pdf.

[40] JEDEC - Failure Mechanisms and Models for Semiconductor Devices. http: / / www . jedec . org/sites/default/files/docs/JEP122F.pdf.

[41] Dawoon Jung, Jeong-UK Kang, Heeseung Jo, Jin-Soo Kim, and Joonwon Lee. Superblock ftl: A superblock-based flash translation layer with a hybrid address translation scheme. ACM Trans. Embed. Comput. Syst., 9(4):40:1-40:41, April 2010.

[42] M. Jung, E.H. Wilson, D. Donofrio, J. Shalf, and M.T. Kandemir. NANDFlashSim: Intrinsic latency variation aware NAND flash memory system modeling and simulation at microarchitecture level. In Mass Storage Systems and Technologies (MSST), 2012 IEEE 28th Symposium on, 2012.

[43] Asim Kadav, Mahesh Balakrishnan, Vijayan Prabhakaran, and Dahlia Malkhi. Differential raid: rethinking raid for ssd reliability. SIGOPS Oper. Syst. Rev., 44:55-59, March 2010.

[44] Jesung Kim, Jong Min Kim, S.H. Noh, Sang Lyul Min, and Yookun Cho. A space-efficient flash translation layer for compactflash systems. Consumer Electronics, IEEE Transactions on, 48(2):366-375, May 2002.

[45] J.K. Kim and et al. A PRAM and NAND flash hybrid architecture for high-performance embedded storage subsystems. In EMSOFT '08: Proceedings of the 8th ACM international conference on Embedded software, pages 31-40, New York, NY, USA, 2008. ACM.

[46] R. Kumar, D.M. Tullsen, and N.P. Jouppi. Core Architecture Optimization of Heterogeneous Chip Multiprocessors. In Proceedings of the International Conference on Parallel Architectures and Compilation Techniques (PACT), September 2006.

[47] L. Larcher, S. Bertulu, and P. Pavan. SILC effects on $E^{2}$ PROM memory cell reliability. Device and Materials Reliability, IEEE Transactions on, 2(1):13 -18, mar 2002. 
[48] J. Lee and et al. A 90-nm CMOS 1.8-V 2-Gb NAND flash memory for mass storage applications. Solid-State Circuits, IEEE Journal of, 38(11):1934-1942, 2003.

[49] S. Lee, D. Park, T. Chung, D. Lee, S. Park, and H. Song. A Log Buffer based Flash Translation Layer Using Fully Associative Sector Translation. IEEE Transactions on Embedded Computing Systems, 6(3), July 2007.

[50] S. Lee, D. Shin, Y. Kim, and J. Kim. LAST: Locality-Aware Sector Translation for NAND Flash Memory-Based Storage Systems. In Proceedings of the International Workshop on Storage and I/O Virtualization, Performance, Energy, Evaluation, and Dependability (SPEED), February 2008.

[51] M. Lenzlinger and E.H. Snow. Fowler-Nordheim tunneling into thermally grown $\mathrm{SiO}_{2}$. Electron Devices, IEEE Transactions on, 15(9):686-686, 1968.

[52] Cheng Li, Philip Shilane, Fred Douglis, Hyong Shim, Stephen Smaldone, and Grant Wallace. Nitro: a capacity-optimized ssd cache for primary storage. In Proceedings of the USENIX Annual Technical Conferences (USENIX), June 2014.

[53] Xiang Luojie and B.M. Kurkoski. An improved analytic expression for write amplification in nand flash. In Computing, Networking and Communications (ICNC), 2012 International Conference on, pages 497-501, Jan 2012.

[54] Marvell SSD Controller. http://www.marvell.com/storage/assets/Marvell_ 88SS9187-001.pdf.

[55] N. Mielke and et al. Recovery effects in the distributed cycling of flash memories. In Reliability Physics Symposium Proceedings, 2006. 44th Annual., IEEE International, pages 29-35, March 2006.

[56] N. Mielke, T. Marquart, Ning Wu, J. Kessenich, H. Belgal, E. Schares, F. Trivedi, E. Goodness, and L.R. Nevill. Bit error rate in nand flash memories. In Reliability Physics Symposium, 2008. IRPS 2008. IEEE International, pages 9-19, May 2008. 
[57] Moazzami, R. and Chenming Hu. Stress-induced current in thin silicon dioxide films. In Electron Devices, IEEE Transactions on, pages 139 -142, December 1992.

[58] Vidyabhushan Mohan, Trevor Bunker, Laura Grupp, Sudhanva Gurumurthi, Mircea R. Stan, and Steven Swanson. Modeling Power Consumption of NAND Flash Memories Using FlashPower. Computer-Aided Design of Integrated Circuits and Systems, IEEE Transactions on, 32(7):1031-1044, July 2013.

[59] Vidyabhushan Mohan, Sudhanva Gurumurthi, and Mircea R. Stan. FlashPower: A Detailed Power Model for NAND flash memory. In Design, Automation Test in Europe Conference Exhibition (DATE), 2010, pages 502 -507, march 2010.

[60] Vidyabhushan Mohan, Taniya Siddiqua, Sudhanva Gurumurthi, and Mircea R. Stan. How I Learned to Stop Worrying and Love Flash Endurance. In Proceedings of the 2nd USENIX conference on Hot topics in storage and file systems, HotStorage'10, Berkeley, CA, USA, 2010. USENIX Association.

[61] Linux Page Table Management. https://www.kernel.org/doc/gorman/html/ understand/understand006.html.

[62] Typical Data Retention for Nonvolatile Memory. http://www.freescale.com/files/ microcontrollers/doc/eng_bulletin/EB618.pdf.

[63] Typical Data Retention for Nonvolatile Memory. http://www.spansion.com/Support/ AppNotes/EnduranceRetention_AN.pdf.

[64] NVSim. http://www.rioshering.com/nvsimwiki/index.php?title=Main_Page.

[65] OCZ Deneva - eMLC based SSD. http://www.oczenterprise.com/downloads/ solutions/ocz-deneva2-c-mlc-2.5in_Product_Brief.pdf.

[66] Jian Ouyang, Shiding Lin, Zhenyu Hou, Peng Wang, Yong Wang, and Guangyu Sun. Active ssd design for energy-efficiency improvement of web-scale data analysis. In Proceedings 
of the 2013 International Symposium on Low Power Electronics and Design, ISLPED '13, pages 286-291, Piscataway, NJ, USA, 2013. IEEE Press.

[67] Jian Ouyang, Shiding Lin, Song Jiang, Zhenyu Hou, Yong Wang, and Yuanzheng Wang. Sdf: Software-defined flash for web-scale internet storage systems. In Proceedings of the 19th International Conference on Architectural Support for Programming Languages and Operating Systems, ASPLOS '14, pages 471-484, New York, NY, USA, 2014. ACM.

[68] Yangyang Pan, Guiqiang Dong, Qi Wu, and Tong Zhang. Quasi-Nonvolatile SSD: Trading Flash Memory Nonvolatility to Improve Storage System Performance for Enterprise Applications. In 2012 IEEE 17th International Symposium on High Performance Computer Architecture (HPCA), feb. 2012.

[69] E. Pinheiro, W-D. Weber, and L.A. Barroso. Failure Trends in a Large Disk Drive Population. In Proceedings of the USENIX Conference on File and Storage Technonologies (FAST), February 2007.

[70] PMC SSD Controller. http://pmcs.com/products/storage/flashtec_nvme_ controllers/pm8604/.

[71] Qi Wu and Guiqiang Dong and Tong Zhang. Exploiting Heat-Accelerated Flash Memory Wear-Out Recovery to Enable Self-Healing SSDs. In Proceedings of the 3rd USENIX conference on Hot topics in storage and file systems, HotStorage'11, Berkeley, CA, USA, 2011. USENIX Association.

[72] Quality Comparison of SLC, MLC and eMLC, 2011. http://www. flashmemorysummit. com/English/Collaterals/Proceedings/2011/20110809_F2C_Wu.pdf.

[73] Quick Path Interconnect. http://www.intel.com/content/www/us/en/io/ quickpath-technology/quick-path-interconnect-introduction-paper.html. 
[74] Ren-Shuo Liu and Chia-Lin Yang, and Wei Wu. Optimizing NAND Flash-Based SSDs via Retention Relaxation. In Proceedings of the 8th USENIX conference on File and storage technologies, FAST'12, 2012.

[75] S. Kavalanekar and et al. Characterization of storage workload traces from production Windows servers. In Proceedings of the IEEE International Symposium on Workload Characterization (IISWC), pages 119-128, October 2008.

[76] Koji Sakui. Intel Corporation/Tohoku University, May 2009. Private Correspondence.

[77] Samsung corporation. K9XXG08XXM flash memory specification. K9XXG08XXM Datasheet.

[78] Samsung V-NAND. http://www.samsung.com/global/business/semiconductor/ product/vnand/overview.

[79] SandForce SSD Controller. http://www.seagate.com/products/ solid-state-flash-storage/flash-controllers/enterprise-flash-controller.

[80] SanDisk Enterprise Solutions. http://www.sandisk.com/enterprise.

[81] SanDisk Enterprise SSDs. http://www.sandisk.com/enterprise/sata-ssd/.

[82] S. Sankar, M. Shaw, and K. Vaid. Impact of temperature on hard disk drive reliability in large datacenters. In Dependable Systems Networks (DSN), 2011 IEEE/IFIP 41st International Conference on, pages $530-537$, june 2011.

[83] H. Schmidt and et al. Tid and see tests of an advanced 8 gbit nand-flash memory. In Radiation Effects Data Workshop, 2008 IEEE, pages 38 -41, 2008.

[84] E. Sharon, I. Alrod, and S. Litsyn. Multi-phase ecc encoding using algebraic codes, February 4 2014. US Patent 8,645,789.

[85] J. Shin, V. Zyuban, P. Bose, and T. Pinkston. A Proactive Wearout Recovery Approach of Exploiting Microarchitectural Redundancy to Extend Cache SRAM Lifetime. In Proceed- 
Bibliography

ings of the International Symposium on Computer Architecture (ISCA), pages 353-362, June 2008.

[86] Clinton W. Smullen, IV, Anurag Nigam, Sudhanva Gurumurthi, and Mircea R. Stan. The STeTSiMS STT-RAM simulation and modeling system. In Proceedings of the International Conference on Computer-Aided Design, pages 318-325. IEEE Press, 2011.

[87] Smullen, Clint and Mohan, Vidyabhushan and Nigam, Anurag. and Gurumurthi, Sudhanva and Stan, Mircea R. Relaxing non-volatility for fast and energy-efficient STT-RAM caches. In High Performance Computer Architecture (HPCA), 2011 IEEE 17th International Symposium on, pages 50-61. IEEE, 2011.

[88] Clinton Wills Smullen IV. Designing Giga-scale Memory Systems with STT-RAM. PhD thesis, University of Virginia, 2011.

[89] Gokul Soundararajan, Vijayan Prabhakaran, Mahesh Balakrishnan, and Ted Wobber. Extending ssd lifetimes with disk-based write caches. In Proceedings of the 8th USENIX conference on File and storage technologies, FAST'10, pages 8-8, Berkeley, CA, USA, 2010. USENIX Association.

[90] K.D. Suh and et al. A 3.3V $32 \mathrm{Mb}$ NAND flash memory with incremental step pulse programming scheme. Solid-State Circuits, IEEE Journal of, 30(11):1149-1156, 1995.

[91] K. Takeuchi, T. Tanaka, and T. Tanzawa. A multipage cell architecture for high-speed programming multilevel NAND flash memories. Solid-State Circuits, IEEE Journal of, 33(8):1228-1238, 1998.

[92] T. Tanaka and et al. A quick intelligent page-programming architecture and a shielded bitline sensing method for 3V-only nand flash memory. Solid-State Circuits, IEEE Journal of, 29(11):1366-1373, 1994.

[93] Application Report: MSP430 Flash Memory Characteristics. http://focus.ti.com/lit/an/slaa334a/slaa334a.pdf. 
[94] A. Tiwari and J. Torrellas. Facelift: Hiding and Slowing Down Aging in Multicores. In Proceedings of the International Symposium on Microarchitecture (MICRO), November 2008.

[95] Devesh Tiwari, Simona Boboila, Sudharshan S Vazhkudai, Youngjae Kim, Xiaosong Ma, Peter Desnoyers, and Yan Solihin. Active flash: towards energy-efficient, in-situ data analytics on extreme-scale machines. In FAST, pages 119-132, 2013.

[96] Toshiba-Sandisk 3D NAND. http://goo.gl/vQqwsL.

[97] Vidyabhushan Mohan, Sriram Sankar and Sudhanva Gurumurthi. reFresh SSDs: Enabling High Endurance, Low Cost Flash in Datacenters. Technical Report CS-2012-05, Department of Computer Science, University of Virginia, Charlottesville, 2012.

[98] Kashi Venkatesh Vishwanath and Nachiappan Nagappan. Characterizing cloud computing hardware reliability. In Proceedings of the 1st ACM symposium on Cloud computing, SoCC '10, pages 193-204, New York, NY, USA, 2010. ACM.

[99] David Tawei Wang. Modern DRAM Memory Systems: Performance analysis and a high performance, power-constrained DRAM scheduling algorithm. $\mathrm{PhD}$ thesis, University of Maryland, College Park, 2005.

[100] S.J.E. Wilton and N.P. Jouppi. Cacti: an enhanced cache access and cycle time model. SolidState Circuits, IEEE Journal of, 31(5):677-688, 1996.

[101] Cong Xu, Xiangyu Dong, N.P. Jouppi, and Yuan Xie. Design implications of memristorbased rram cross-point structures. In Design, Automation Test in Europe Conference Exhibition (DATE), 2011, pages $1-6,2011$.

[102] E. Yaakobi, Jing Ma, L. Grupp, P.H. Siegel, S. Swanson, and J.K. Wolf. Error characterization and coding schemes for flash memories. In GLOBECOM Workshops (GC Wkshps), 2010 IEEE, pages $1856-1860,2010$. 
[103] R. Yamada and et al. A novel analysis method of threshold voltage shift due to detrap in a multi-level flash memory. In Symposium on VLSI Technology, Digest of Technical Papers, 2001.

[104] H. Yang and et al. Reliability issues and models of sub-90nm NAND flash memory cells. In International Conference on Solid-State and Integrated Circuit Technology, 2006.

[105] Qing Yang and Jin Ren. I-cash: Intelligently coupled array of ssd and hdd. In High Performance Computer Architecture (HPCA), 2011 IEEE 17th International Symposium on, pages $278-289$, feb. 2011. 